\title{
APROXIMAÇÕES PARA RESTRIÇÕES DO PROBLEMA DE STEINER EM GRAFOS
}

\author{
Fábio Henrique Viduani Martinez
}

\author{
Tese apresentada ao \\ Instituto de Matemática e Estatística da \\ Universidade de São Paulo para \\ obtenção do grau de doutor em \\ Ciência da Computação
}

Área de Concentração: Ciência da Computação

Orientador: Prof. Dr. José Augusto Ramos Soares

Durante o desenvolvimento deste trabalho o autor recebeu apoio financeiro da CAPES

São Paulo, agosto de 2004 


\section{APROXIMAÇÕES PARA RESTRIÇÕES DO PROBLEMA DE STEINER EM GRAFOS}

Este exemplar corresponde à redação final da tese devidamente corrigida e defendida por Fábio Henrique Viduani Martinez e aprovada pela comissão julgadora.

São Paulo, 19 de agosto de 2004.

Banca examinadora:

- Prof. Dr. José Augusto Ramos Soares (orientador) (IME-USP)

- Prof. Dr. Carlos Eduardo Ferreira (IME-USP)

- Prof. Dr. Flávio Keidi Miyazawa (IC-UNICAMP)

- Prof. Dr. Kátia Silva Guimarães (CIn-UFPE)

- Prof. Dr. Marcelo Henriques de Carvalho (DCT-UFMS)

Suplentes:

- Prof. Dra. Cristina Gomes Fernandes (IME-USP)

- Prof. Dr. João Meidanis (IC-UNICAMP) 
para Valguima

"Sim, me leva para sempre, (...)

Me ensina a não andar com os pés no chão Para sempre é sempre por um triz

$\mathrm{Ai}$, diz quantos desastres tem na minha mão Diz se é perigoso a gente ser feliz

(...)

E se eu pudesse entrar na sua vida"

Chico Buarque / Edu Lobo (1982) 


\section{Agradecimentos}

Estes agradecimentos não são meramente formais. Embora não consiga, tento expressá-los com todo sentimento.

Um agradecimento mais que especial ao meu orientador, professor José Augusto Ramos Soares. Houve dois momentos durante esse período que eu quero deixar registrados. O Zé Augusto 'estudou' comigo Análise de Algoritmos, quando prestei o exame preliminar pela segunda vez. E no final, quando tudo parecia perdido, quando tudo eram trevas, ele ajudou demais, esteve mais que presente. $\mathrm{E}$ ainda minhas desculpas pelos erros, pelo sentimento de derrota que muitas vezes me acompanhou, pela ânsia em corresponder, pelas muitas e muitas histórias que teve de ouvir, pelo eastern daylight time, entre outros.

Ao professor José Coelho de Pina Júnior, pela disponibilidade, pela receptividade, pelos ensinamentos e por todas as conversas.

A todos os professores e funcionários do DCT/UFMS, pelo apoio e pelo sacrifício durante minha ausência.

À dra. Flávia, com admiração pelo seu trabalho e pela grande ajuda em meu autoconhecimento.

Aos funcionários do IME/USP, em especial ao Joel, Marcos, Francisco, Jovita, Dalvina, Jahi, Rinildo (Morfeu), Nilson, Sérgio, Pinho, Adenilza, Marina, Sandro, João, pela simpatia, presteza e cordialidade.

Ao pessoal da pós, Cecília, Pablo, Bárbara, Maité, Nandinho, Alice, Raul, Zé, Irene, Samuel, Mário, Major, Suzi, Luciana, Silvia, Délhi, Ronaldo, Paulo, David, Rogério, Fábio Luba, Leonardo, pela amizade e por me fazerem sentir que estávamos no mesmo barco. Ao pessoal da sala 141B, Fernanda, Neusa, Wellington, Gladys, Caio, por tornarem agradável nosso pequeno e desconfortável espaço, pela troca, pela força e pelas conversas. Ao pessoal do $304 \mathrm{G}$ do CRUSP, que me recebeu na chegada e na partida. Um agradecimento ao Mário Leston, pela paciência em sempre me ouvir e pela troca de experiências. Também ao Renato, pelas conversas e pela paciência especialmente em SO. E ao super Elói, irmão de tese, pela amizade, pelo exemplo de força e pelas conversas. 
A toda minha família em Campo Grande. Ao patriarca Orlando Viduani, meu avô, pela doçura e pelas histórias. À minha avó Flávia (in memoriam). Aos meus queridos pais, pela compreensão em minhas tantas ausências e pela força constante. À minha irmã, pela luta em estar sempre próxima e pelo incentivo. E à minha querida sobrinha Anna Carolina, pelo sorriso e carinho angelical.

À D. Daguimar, pelo exemplo, força, carinho, acolhimento e pela enorme paciência em sempre me ajudar.

Eu estive envolvido por amigos maravilhosos nesse período, minha camada protetora antiimpactos. Um enorme agradecimento à Fátima, pela amizade incondicional, pelo cuidado, pela disponibilidade e pelas conversas. Ao Marco Aurélio, pela força nos momentos que precisei e ainda preciso, pela imensa amizade de longa data. Ao Luiz Carlos (Charlie Brown), pelo companheirismo e força; e à Débora Rozante. Ao Claus, pela disposição em sempre ajudar e pelo cuidado. Ao Jair e à Karin, pelo acolhimento e pela presença. À Lighia, pela proteção e carinho. À Eliany pela ajuda e carinho; e ao Rafael. Ao Marcelo e Poliana, pela amizade e presença constante, mesmo lá da Filadélfia. Ao Sérgio, pela amizade descontraída e pelo acolhimento. Ao grande Said, pela paciência e pela força. À Lucia Ikemoto e ao Ricardo, pela lucidez, pelas palavras e pelo acolhimento. Aos casais com filhinhos, Marcelo Lauretto, Renata, Lucas e Camila; Fran, Cris e Rafael; Ivone, Juliano e Natália; Renata Wasserman, Marcelo e Laura; pelo carinho e pelas horas divertidas.

E um agradecimento muito especial à Val, pelo amor, dedicação e carinho, pelo tempo maravilhoso que trilhamos o mesmo caminho, por ter revelado em mim todo amor que eu consigo sentir, pelos melhores anos da minha vida. Não tem onde caiba o que é por você... 
Sigmund Freud freqüentemente observava que grandes revoluções na história da ciência tinham pelo menos uma característica comum e irônica: arrasavam com a arrogância humana, um pedestal após outro, com a nossa convicção prévia sobre nossa própria importância. Nos três exemplos de Freud, Copérnico moveu nossa casa do centro para a periferia, Darwin então nos relegou a "descendentes de um mundo animal" e, finalmente (em uma das menos modestas sentenças da história intelectual), o próprio Freud descobriu o inconsciente e explodiu de vez o mito de uma mente completamente racional. Neste crucial e sábio sentido, a revolução darwiniana permanece dolorosamente incompleta já que, mesmo apesar de o pensamento humano aceitar o fato da evolução, muitos de nós não estamos ainda dispostos a abandonar a visão confortável que evolução significa (ou pelo menos incorpora um princípio central de) progresso, definido a ter a aparência de algo que a consciência humana determina e que é virtualmente inevitável ou pelo menos predizível. O pedestal não será quebrado até abandonarmos o progresso ou a complexificação como um princípio central e considerarmos a forte possibilidade que o Homo sapiens é nada mais que um pequeníssimo e bastante recente galho na enorme ramificação da árvore da vida - um pequeno broto que certamente não apareceria uma segunda vez se pudéssemos replantar a árvore a partir de sua semente e a deixássemos crescer novamente.

\section{Stephen Jay Gould}

The Evolution of Life on Earth, Scientific American, 271(4): página 94, 1994. 


\section{Resumo}

No problema de Steiner em grafos é dado um grafo completo com custos nas arestas e um subconjunto de vértices chamados terminais e queremos encontrar uma árvore de menor custo que conecte todos os terminais. Este trabalho aborda restrições desse problema. Os problemas abordados têm aplicações em construção de árvores filogenéticas em biologia, roteamento local ou global no projeto de placas VLSI, transporte e telecomunicações, bem como são úteis para se estabelecer a complexidade computacional para os problemas sem restrições.

O primeiro problema abordado é o da árvore de Steiner de terminais folhas, onde exigimos que os terminais sejam folhas na árvore resultante. O segundo problema é o da árvore de Steiner de terminais folhas com custos 1 ou 2 nas arestas. Apresentamos algoritmos de aproximação que melhoram as razões de aproximação previamente conhecidas para esses problemas.

Propomos também uma nova variante do problema, na qual uma permutação dos vértices terminais também é dada como entrada. Queremos encontrar agora uma árvore de Steiner de menor custo que respeite a permutação dada. Dizemos que a árvore respeita a permutação se sempre que terminais $r_{1}, r_{2}, r_{3}$ e $r_{4}$ aparecem nesta ordem na permutação, os caminhos na árvore entre $r_{1}$ a $r_{3}$ e entre $r_{2}$ a $r_{4}$ têm pelo menos um vértice em comum. Mostramos que árvores k-restritas são aproximações para esse problema na mesma razão que o são em geral para o problema de Steiner em grafos. E mostramos um algoritmo que encontra em tempo polinomial uma árvore k-restrita ótima para esta versão do problema. 


\begin{abstract}
In the Steiner problem in graphs a complete graph with edge costs and a subset of vertices called terminals are given and we want to find a minimum cost tree that connects all the terminals. This work deals with restricted versions of this problem. These versions have applications in the construction of phylogenetic trees in biology, local or global routing in the VLSI design, transportation and telecommunications. Furthermore, complexity results for the general problem can be immediately established by any complexity result obtained for restricted versions of the problem.

The first problem we study is the terminal Steiner tree problem, where terminals must be leaves in the resultant tree. The second problem is the terminal Steiner tree problem with edge costs 1 or 2 . We present algorithms that improve the previously known approximation ratios for these problems.

We also propose a new version of the problem, in which a permutation of the terminals is also given. We want to determine a minimum cost Steiner tree that respects the given permutation. We say that a tree respects the permutation if whenever terminals $r_{1}, r_{2}, r_{3}$, and $r_{4}$ appears in this order in the permutation, the paths in the tree between $r_{1}$ and $r_{3}$ and between $r_{2}$ and $r_{4}$ share at least one vertex. We show that k-restricted Steiner trees approximate this problem with the same ratio that they approximate the Steiner tree problem in general. We also present a polynomial time algorithm that finds an optimum $k$-restricted Steiner tree for this version of the problem.
\end{abstract}




\section{Sumário}

1 Introdução 1

1.1 Algoritmos de aproximação . . . . . . . . . . . . . . . . . . . 2

1.2 Complexidade computacional para problemas de otimização . . . . . . . . . . 3

1.3 Problemas de Steiner em grafos . . . . . . . . . . . . . . . . 5

1.3.1 Problema da árvore de Steiner em grafos . . . . . . . . . . . . . 5

1.3.2 Problema da árvore de Steiner dos terminais folhas . . . . . . . . . . . 9

1.3.3 Problema da árvore de Steiner com ordenação nos terminais . . . . . . . . 11

2 Problema TST 13

2.1 Uma 3,10-aproximação para o problema TST . . . . . . . . . . . . . . 14

2.2 Uma 2,76-aproximação para o problema TST . . . . . . . . . . . . . . . 16

2.2.1 Algoritmo Árvore $\ldots \ldots \ldots \ldots \ldots$. . . . . . . . . . . . 17

2.2 .2 Algoritmo Árvore $\ldots \ldots \ldots \ldots \ldots$

2.2 .3 Algoritmo Terminal . . . . . . . . . . . . . . . . 25

2.3 Uma 2,52-aproximação para o problema TST . . . . . . . . . . . . . . . . . 27

2.4 APX-completude para o problema TST . . . . . . . . . . . . . . . 31

3 Problema TST $_{1,2} \quad 35$

3.1 Uma 2-aproximação para o problema $\mathrm{TST}_{1,2} \ldots \ldots \ldots \ldots \ldots$

3.2 Uma $8 / 5$-aproximação para o problema $\mathrm{TST}_{1,2} \ldots \ldots \ldots$. . . . . . . . 37

3.3 Uma $17 / 12$-aproximação para o problema $\mathrm{TST}_{1,2} \ldots \ldots \ldots \ldots \ldots \ldots$

3.3 .1 Reduçōes .......................... . . 44

3.3.2 Algoritmo Empacota . . . . . . . . . . . . . . . . . . . 47

3.3.3 Algoritmo Terminal Al, $_{1} \ldots \ldots \ldots \ldots \ldots \ldots \ldots$ 
3.4 APX-completude para o problema $\mathrm{TST}_{1,2} \ldots \ldots \ldots \ldots \ldots$

4 Problema STO $\quad 57$

4.1 Razão k-Steiner para árvores de Steiner com ordenação nas folhas . . . . . . . . 57

4.2 Uma $\rho_{k}$-aproximação para o problema STO . . . . . . . . . . . . . . . 68

$\begin{array}{lll}5 & \text { Conclusão } & 73\end{array}$

$\begin{array}{ll}\text { Referências Bibliográficas } & 75\end{array}$ 


\section{Capítulo 1}

\section{Introdução}

Este trabalho aborda restrições do problema de Steiner em grafos: o problema da árvore de Steiner de terminais folhas, o problema da árvore de Steiner de terminais folhas com custos 1 ou 2 nas arestas e o problema da árvore de Steiner com uma permutação para seus terminais. No primeiro problema, dado um grafo com custos nas arestas e um subconjunto de vértices chamados terminais, queremos encontrar um subgrafo conexo que contém os vértices terminais e, além disso, que esses terminais tenham grau 1; tal subgrafo é chamado de árvore de Steiner de terminais folhas e os vértices terminais são folhas nessa árvore. O segundo problema tem o mesmo objetivo, mas restringe o custo das arestas do grafo para 1 ou 2. E, finalmente, no último problema que abordamos, queremos encontrar uma árvore de Steiner que respeita uma ordenação para seus terminais.

Árvores de Steiner de terminais folhas têm um papel importante em aplicaçōes como a construção de árvores filogenéticas em biologia (Lu et al., 2003), roteamento local ou global no projeto de placas VLSI (Lin \& Xue, 2002; Fuchs, 2003; Drake \& Hougardy, 2004), transporte (Lin \& Xue, 2002; Fuchs, 2003) e telecomunicações (Lin \& Xue, 2002; Fuchs, 2003). Árvores de Steiner que respeitam ordenações impostas são também importantes na construção de árvores filogenéticas em biologia.

O problema da árvore de Steiner de terminais folhas é computacionalmente difícil (Lin \& Xue, 2002; Drake \& Hougardy, 2004) e trabalhos anteriores têm apresentado aproximações para o problema (Lin \& Xue, 2002; Fuchs, 2003; Chen et al., 2003; Drake \& Hougardy, 2004). Da mesma forma, o problema da árvore de Steiner de terminais folhas com custos 1 ou 2 nas arestas é difícil e uma aproximação para o problema foi proposta (Lu et al., 2003). Nosso trabalho resultou no projeto de dois algoritmos de aproximação para o problema da árvore de Steiner de terminais folhas que melhoram a razão de aproximação estabelecida em trabalhos anteriores, no projeto de um algoritmo de aproximação que melhora a razão de aproximação proposta anteriormente para o problema da árvore de Steiner de terminais folhas com custos 1 ou 2 nas arestas e na construção de um algoritmo de aproximação para o problema da árvore de Steiner com uma ordenação para os terminais.

As seções a seguir apresentam fundamentos e a notação adotada neste trabalho. 


\subsection{Algoritmos de aproximação}

Um algoritmo de aproximação de tempo polinomial projetado para solucionar um problema de otimização estabelece um compromisso entre sua eficiência e uma solução ótima do problema: por um lado, como trabalhamos em geral com problemas de otimização que são computacionalmente difíceis, o algoritmo de aproximação se apresenta vantajoso por computar eficientemente uma solução para o problema; por outro lado, a solução apresentada pode não ser a solução ótima para o problema, mas o algoritmo garante que a solução encontrada mantém uma relação bem estabelecida com a solução ótima e não está muito distante dela.

A seguir formalizamos as definições de problemas de otimização e de algoritmos de aproximação e fixamos uma notação, baseados nos textos de Fernandes et al. (2001) e de Vazirani (2001).

Um problema de otimização (combinatória) $\Pi$ consiste de:

(i) um conjunto de instâncias $\mathcal{I}_{\Pi}$, chamado domínio de $\Pi$;

(ii) um conjunto de soluções viáveis $\operatorname{Sol}_{\Pi}(I)$, para cada instância $I \in \mathcal{I}_{\Pi}$;

(iii) uma função objetivo val ${ }_{\Pi}$ que atribui um número val ${ }_{\Pi}(S)$ para cada solução viável $S$;

(iv) a definição de que $\Pi$ é um problema de minimização ou um problema de maximização.

Um problema de minimização (maximização) busca por soluções viáveis de valor mínimo (máximo) para cada instância. Chamamos esse valor de valor ótimo e o valor de qualquer das soluções ótimas de uma instância I é denotado por opt(I). Quando uma instância é clara no contexto, usamos simplesmente opt no lugar de opt(I) e fazemos o mesmo com as definições que se seguem.

Como mencionamos antes, uma estratégia para tentar superar a dificuldade imposta por problemas de otimização NP-difíceis é construir algoritmos que produzam soluções viáveis que estão em algum sentido próximas das soluções ótimas. Mais formalmente, seja $\Pi$ um problema de minimização (maximização) e seja $A$ um algoritmo que, para toda instância I do problema, devolve uma solução viável $A(I) \in \operatorname{Sol}_{\Pi}(I)$ de I. Então, se val $(A(I)) \leqslant \alpha \operatorname{opt}(I)$ $(\operatorname{val}(A(I)) \geqslant \alpha \operatorname{opt}(I))$ para toda instância I, dizemos que $A$ é uma $\alpha$-aproximação para o problema. Dizemos que $\alpha$ é uma razão de aproximação do algoritmo $A, \operatorname{com} \alpha \geqslant 1(\alpha \leqslant 1)$. Um algoritmo de aproximação é uma $\alpha$-aproximação para algum $\alpha$. É necessário ainda que o algoritmo $A$ seja um algoritmo de tempo polinomial, isto é, que seu consumo de tempo seja limitado por um polinômio no tamanho da instância I. Por tamanho de uma instância I queremos dizer o comprimento da cadeia de caracteres que representa I e denotamos esse número por $\langle\mathrm{I}\rangle$.

Observe que para determinar a razão de aproximação de um algoritmo de aproximação para um problema NP-difícil, o custo de uma solução viável produzida pelo algoritmo deve ser comparado com o custo de uma solução ótima do problema. No entanto, para um problema desse tipo, computar o valor da solução ótima é tão difícil quanto computar a própria solução 
ótima e então, para demonstrar que um algoritmo é uma $\alpha$-aproximação, é fundamental obter eficientemente boas delimitações do valor das soluções ótimas.

Após o projeto de um algoritmo de aproximação A para um problema $\Pi$ e da determinação de sua razão de aproximação $\alpha$ é importante verificar se podemos melhorar a razão $\alpha$ ou se existe um exemplo para o qual o valor da solução encontrada está distante exatamente $\alpha$ vezes do valor de uma solução ótima. Uma família infinita de instâncias que mostra que a análise de um algoritmo de aproximação é justa é chamada um exemplo justo.

\subsection{Complexidade computacional para problemas de otimização}

Problemas de decisão são classificados em termos de sua complexidade, de acordo com sua dificuldade computacional, e isso se traduz tecnicamente nas classes P e NP. Problemas de otimização são classificados conforme seu grau de aproximabilidade por algoritmos polinomiais nas classes PO, FPTAS, PTAS, APX e NPO, as quais serão definidas a seguir.

Seja $\Pi$ um problema de otimização definido por um conjunto de instâncias, um conjunto Sol(I) de soluções viáveis associado a cada instância I e uma função val que associa a cada instância I e solução $S$ um valor racional não-negativo val( $I, S)$. O objetivo então é encontrar uma solução viável de valor ótimo (mínimo ou máximo).

A classe de problemas NPO é a extensão da classe NP para problemas de otimização onde:

(i) existe uma função polinomial $p$ tal que $\langle S\rangle \leqslant p(\langle I\rangle)$ para toda instância I do problema e toda solução viável S de I;

(ii) existe um algoritmo polinomial que decide se uma dada cadeia de caracteres é uma representação válida de uma instância do problema;

(iii) existe um algoritmo polinomial que decide se um dado objeto é solução viável de uma instância do problema;

(iv) existe um algoritmo polinomial que calcula val(I,S), dados I e S.

A classe PO é formada pelo conjunto de problemas em NPO para os quais existe um algoritmo exato polinomial e é a extensão natural da classe $\mathrm{P}$ para problemas de otimização. A classe APX é formada pelos problemas em NPO para os quais existe uma $\alpha$-aproximação polinomial para alguma constante $\alpha$.

Um esquema de aproximação para um problema de otimização é um algoritmo $A$ que recebe um número racional positivo $\varepsilon$ e uma instância I e devolve uma solução viável $A(\varepsilon, I)$ com erro relativo de no máximo $\varepsilon$, ou seja,

$$
(1-\varepsilon) \operatorname{opt}(I) \leqslant \operatorname{val}(I, A(\varepsilon, I)) \leqslant(1+\varepsilon) \operatorname{opt}(I) .
$$

Se o problema em questão é um problema de maximização, então a desigualdade esquerda é a que interessa, com $0<\varepsilon<1$, e se o problema é de minimização, é a desigualdade direita. 
Dizemos que o algoritmo $A$ é um esquema de aproximação polinomial se o algoritmo $A(\varepsilon, \cdot)$ é polinomial para todo $\varepsilon$ fixo. Quando a quantidade de tempo consumida pelo algoritmo é limitada por uma função polinomial p em $\langle I\rangle$ e $\varepsilon$, então o algoritmo $A$ é chamado de esquema de aproximação plenamente polinomial.

Dessa forma, a classe PTAS de problemas de otimização é composta pelos problemas em NPO que possuem um esquema de aproximação polinomial e a classe FPTAS pelos que admitem um esquema de aproximação plenamente polinomial. Dessas definições, temos que

$$
\mathrm{PO} \subseteq \mathrm{FPTAS} \subseteq \mathrm{PTAS} \subseteq \mathrm{APX} \subseteq \mathrm{NPO}
$$

Os diferentes níveis de dificuldade que se revelam na tentativa de construção de algoritmos de aproximação para diferentes problemas de otimização, somados à dificuldade em melhorar razões de aproximação para algoritmos propostos, são formalizados através das relações entre as classes de aproximação que aqui foram recém-definidas. Como não é nossa intenção neste texto apresentar as demonstrações necessárias, apresentamos a seguir os resultados relativos a essas classes em um único teorema, observando que esta não é a maneira usual que tais resultados aparecem na literatura. Leitores interessados devem consultar os livros-texto de Ausiello et al. (1999), Fernandes et al. (2001) e Vazirani (2001).

\section{TEOREMA 1.1}

(i) $\mathrm{Se} \mathrm{P}=\mathrm{NP}$ então $\mathrm{PO}=\mathrm{NPO}$

(ii) $\mathrm{Se} \mathrm{PO}=$ FPTAS então $\mathrm{P}=\mathrm{NP}$.

(iii) Se FPTAS $=$ PTAS então $\mathrm{P}=\mathrm{NP}$.

(iv) Se PTAS $=\mathrm{APX}$ então $\mathrm{P}=\mathrm{NP}$.

(v) Se $\mathrm{APX}=\mathrm{NPO}$ então $\mathrm{P}=\mathrm{NP}$.

O conceito de completude, como conhecido na classe NP, pode ser estendido para problemas de otimização através do uso de uma redução que preserva a polinomialidade e a razão de aproximação.

Uma AP-redução de um problema de otimização $\Pi$ para um problema de otimização $\Pi^{\prime}$ é uma tripla $(f, g, \beta)$ onde $f$ e $g$ são algoritmos e $\beta$ é um número racional positivo tais que:

(AP1) f recebe um número racional positivo $\delta$ e uma instância I de $\Pi$, e devolve uma instância $f(\delta, I)$ de $\Pi^{\prime}$

(AP2) g recebe um número racional positivo $\delta$, uma instância $I$ de $\Pi$ e um elemento $S^{\prime}$ de Sol $(f(\delta, I))$, e devolve $g\left(\delta, I, S^{\prime}\right)$ em Sol $(I)$;

(AP3) para todo número racional positivo $\delta$, os algoritmos $f(\delta, \cdot)$ e $g(\delta, \cdot, \cdot)$ são polinomiais; e 
(AP4) para toda instância I de $\Pi$, todo número racional positivo $\delta$, e todo $S^{\prime}$ em $\operatorname{Sol}(f(\delta, I))$, vale que se

$$
\left.(1-\delta) \operatorname{opt}(f(\delta, I)) \leqslant \operatorname{val}(f(\delta, I)), S^{\prime}\right) \leqslant(1+\delta) \operatorname{opt}(f(\delta, I))
$$

então

$$
(1-\beta \delta) \text { opt }(I) \leqslant \operatorname{val}\left(I, g\left(\delta, I, S^{\prime}\right)\right) \leqslant(1+\beta \delta) \text { opt }(I) .
$$

Usamos a notação $\Pi \leqslant A P \Pi^{\prime}$ para denotar a existência de uma AP-redução de $\Pi$ a $\Pi^{\prime}$. Dizemos que $\Pi$ pode ser AP-reduzido a $\Pi^{\prime}$ significando que $\Pi \leqslant \mathrm{AP} \Pi^{\prime}$.

Os resultados a seguir são herança de resultados equivalentes para as classes P e NP.

TEOREMA $1.2 \mathrm{Se} \Pi_{1} \leqslant \mathrm{AP} \Pi_{2}$ e $\Pi_{2} \leqslant \mathrm{AP} \Pi_{3}$, então $\Pi_{1} \leqslant \mathrm{AP} \Pi_{3}$.

TEOREMA 1.3 Se $\Pi$ está em NPO, $\Pi^{\prime}$ está em APX e $\Pi \leqslant \mathrm{AP} \Pi^{\prime}$, então $\Pi$ está em APX.

O próximo teorema mostra que a AP-redução preserva a existência de um esquema de aproximação polinomial.

TEOREMA 1.4 Se $\Pi$ está em NPO, $\Pi^{\prime}$ está em PTAS $e \Pi \leqslant \mathrm{AP} \Pi^{\prime}$ então $\Pi$ está em PTAS.

Um problema $\Pi$ em APX é APX-completo se cada problema em APX pode ser AP-reduzido a $\Pi$. Um problema $\Pi$, não necessariamente em APX, é APX-difícil se a existência de um esquema de aproximação polinomial para $\Pi$ implica em $P=N P$. Observe que, se PTAS $\neq A P X$, então todo problema APX-completo está em APX $\backslash$ PTAS.

Um problema $\Pi$ em NPO é NPO-completo se cada problema em NPO pode ser APreduzido a $\Pi$. Note que, se APX $\neq$ NPO, então todo problema NPO-completo está em $\mathrm{NPO} \backslash \mathrm{APX}$.

O limiar de aproximação de um problema de minimização (maximização) é o maior (menor) limitante inferior (superior) de todos os $\alpha$ para os quais existe uma $\alpha$-aproximação polinomial para o problema.

\subsection{Problemas de Steiner em grafos}

Descrevemos nesta seção o problema da árvore de Steiner em grafos e três restrições para esse problema, que são o problema da árvore de Steiner dos terminais folhas, o problema da árvore de Steiner dos terminais folhas com arestas de custo 1 ou 2 e o problema da árvore de Steiner com uma ordenação para os terminais.

\subsubsection{Problema da árvore de Steiner em grafos}

Considere um grafo $G$, uma função custo $c: E_{G} \rightarrow \mathbb{Q} \geqslant$ nas arestas de $G$ e um conjunto $R \subseteq V_{G}$ de vértices chamados terminais. Uma árvore de Steiner em $G$ é um subgrafo conexo de $\mathrm{G}$ que contém todos os terminais. A figura 1.1 moștra um exemplo para essa definição. 


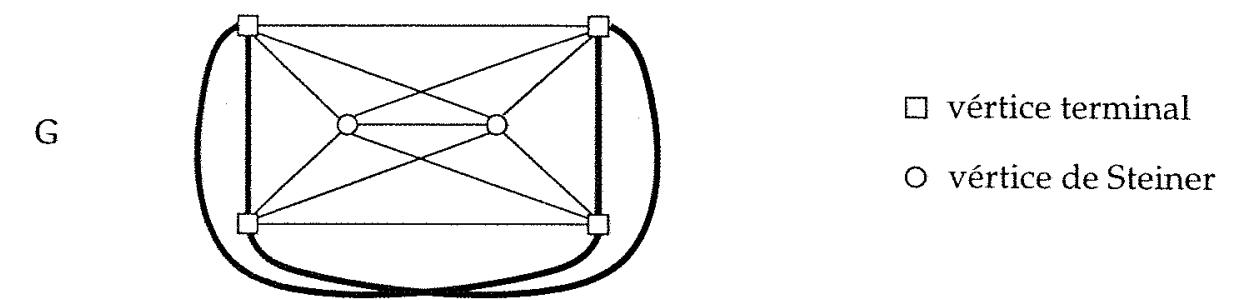

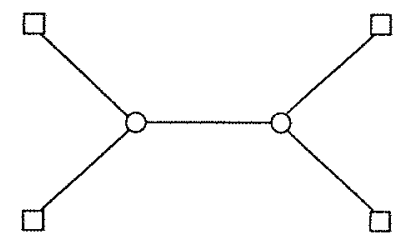

(i)

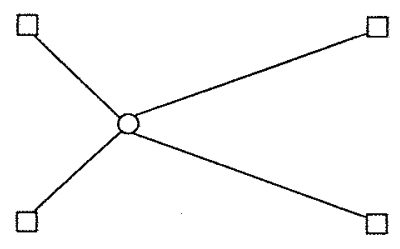

(ii)

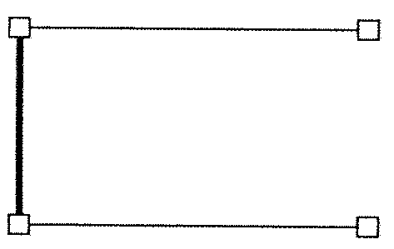

(iii)

Figura 1.1: Um grafo G e árvores de Steiner em G. Arestas finas têm custo 1 e arestas grossas custo 2 . O custo de uma árvore de Steiner mínima em G é 4.

Denotamos por $V_{G}$ e $E_{G}$ os conjuntos de vértices e arestas de $G$, respectivamente. Podemos estender a definição da função custo para conjuntos de arestas fazendo $c(X):=\sum_{X \in X} c(X)$ para qualquer $X \subseteq E_{G}$. Da mesma forma, definimos $c(G)$ para um grafo $G$ como o custo total de suas arestas, isto é, $c(G):=c\left(E_{G}\right)$. Assim, podemos escrever $c(T)$ para o custo de uma árvore de Steiner T.

No problema de Steiner em grafos, dada uma instância G, c e R, queremos encontrar uma árvore de Steiner de custo mínimo no grafo G. Isto é, queremos encontrar uma árvore que conecte todos os vértices de R e cuja soma total dos custos de suas arestas seja mínima. Uma árvore de Steiner de custo mínimo para uma instância é chamada árvore de Steiner mínima.

O problema de Steiner em grafos (Steiner minimum tree problem) é então formalizado da seguinte maneira:

Problema ST $(G, c, R)$ : dado um grafo completo $G$, uma função custo $c$ : $E_{G} \rightarrow$ $\mathbb{Q} \geqslant$ satisfazendo a desigualdade triangular e um conjunto $R \subseteq V_{G}$ de terminais, encontrar uma árvore de Steiner mínima em G.

Vale ainda a observação que trabalhamos com o problema métrico de Steiner em grafos: o grafo $G$ é uma métrica, isto é, $G$ é completo e vale a desigualdade triangular para o custo das arestas de $\mathrm{G}$.

O problema ST tem diversas aplicações e tem sido muito estudado. Bern \& Plassmann (1989) e Arora et al. (1992) mostraram que o problema ST é APX-difícil. O primeiro algoritmo de aproximação para o problema ST foi proposto por Moore, conforme menção de Gilbert \& Pollak (1968), tem razão de aproximação 2 e é conhecido como heurística da árvore geradora de custo mínimo. A idéia desse algoritmo é simplesmente computar uma árvore geradora de custo mínimo para o subgrafo dos terminais da instância do problema. 
A heurística da árvore geradora mínima permaneceu por mais de vinte anos como a melhor aproximação para o problema ST, devido em especial à dificuldade de análise das heurísticas que estavam sendo propostas àquela época. Esse panorama foi mudado a partir do trabalho de Zelikovsky (1993), que propôs a utilização de árvores de Steiner k-restritas no desenvolvimento e análise de algoritmos de aproximação para o problema ST. A partir de então, todos os algoritmos de aproximação propostos para esse problema de alguma forma fazem uso dessas árvores de Steiner k-restritas. Segue a definição dessa estrutura.

Uma árvore de Steiner tal que todos os seus terminais são folhas é chamada uma árvore de Steiner de terminais folhas. Retomaremos essa definição a seguir mas, por agora, observe que árvores de Steiner que não são árvores de Steiner de terminais folhas podem ser decompostas em componentes de terminais folhas pelo compartilhamento de vértices terminais interiores da árvore de Steiner. Veja a figura 1.2.

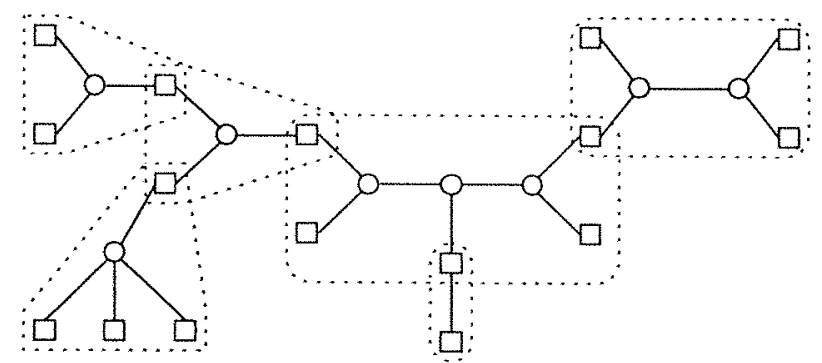

Figura 1.2: Decomposição de uma árvore de Steiner em componentes de terminais folhas.

Sejam $G$ um grafo completo, $c: E_{G} \rightarrow \mathbb{Q} \geqslant$ uma função custo nas arestas de $G$ satisfazendo a desigualdade triangular e $R \subseteq V_{G}$ um conjunto de terminais. Uma árvore de Steiner $k$ restrita para a instância $G, c, R$ e $k$ é uma coleção de componentes de terminais folhas com no máximo $k$ terminais cada uma, que estão conectadas e, juntas, cobrem todo o conjunto de terminais. A figura 1.2 ilustra uma árvore de Steiner 5-restrita e a figura 1.3 mostra outros exemplos de árvores de Steiner k-restritas. Observe que toda árvore de Steiner associada a uma instância G, c e R é uma árvore de Steiner k-restrita para algum k suficientemente grande, digamos $k=|R|$. Em geral, o custo de uma árvore de Steiner k-restrita ótima para uma instância pode ser maior que o custo de uma árvore de Steiner ótima para a mesma instância, como mostra a figura 1.3.

O problema da árvore de Steiner k-restrita de custo mínimo pode ser estabelecido como a seguir.

Problema $\mathrm{kST}(\mathrm{G}, \mathrm{c}, \mathrm{R}, \mathrm{k})$ : dado um grafo completo $\mathrm{G}$, uma função custo $c: E_{G} \rightarrow \mathbb{Q} \geqslant$ satisfazendo a desigualdade triangular, um conjunto $R \subseteq V_{G}$ de terminais e um inteiro positivo $k$, tal que $k \leqslant|R|$, encontrar uma árvore de Steiner k-restrita de custo mínimo em $\mathrm{G}$.

Como a figura 1.3 mostra, se $k$ é grande o suficiente, então intuitivamente uma árvore de Steiner k-restrita ótima é uma boa aproximação para uma árvore de Steiner ótima. Mais preci- 


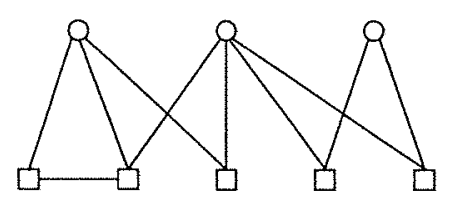

(i)

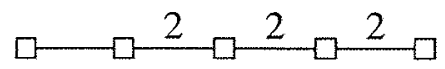

(ii)

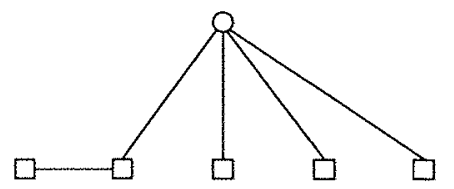

(iv)

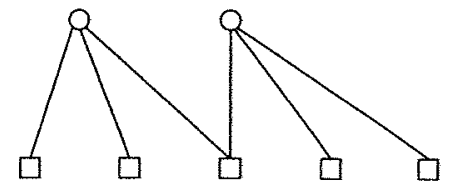

(iii)

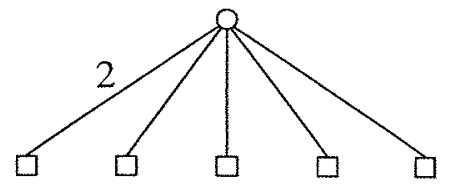

$(v)$

Figura 1.3: (i) Um grafo $\mathrm{G}$ com custos associados às suas arestas, onde são apresentadas apenas as arestas que têm custo $1 \mathrm{e}$ as arestas não apresentadas têm custo 2. (ii) Uma árvore 2-restrita ótima para G, c e R, com custo 7. (iii) Uma árvore 3-restrita ótima com custo 6 para a mesma instância. (iv) Uma árvore de Steiner 4-restrita ótima de custo 5. (v) Uma árvore de Steiner 5-restrita de custo 6; observe que a árvore de Steiner 5-restrita ótima tem custo 5 e é a árvore apresentada em (iv).

samente, a razão $k-S t e i n e r \rho_{k}$ é definida como sendo

$$
\rho_{k}:=\sup _{(G, c, R)}\left\{\frac{\operatorname{opt}(k S T(G, c, R, k))}{\operatorname{opt}(S T(G, c, R))}\right\}
$$

A 2-aproximação estabelecida pela heurística da árvore geradora de custo mínimo, já mencionada anteriormente, faz $\rho_{2}=2$. Zelikovsky (1993) mostrou um limitante superior para $\rho_{3}$ e seu trabalho foi generalizado por Borchers \& Du (1997), que propuseram a seguinte fórmula para $\rho_{k}$.

Teorema 1.5 (Borchers \& Du, 1997) Para $k=2^{r}+s$, com $0 \leqslant s<2^{r}$, temos que

$$
\rho_{k}:=\frac{(r+1) \cdot 2^{r}+s}{r \cdot 2^{r}+s}
$$

Esse resultado implica que

$$
\lim _{k \rightarrow+\infty} \rho_{k}=1 \text {, }
$$

e assim sabemos que árvores de Steiner k-restritas de custo mínimo são boas aproximações para árvores de Steiner de custo mínimo, se k é suficientemente grande. No entanto, a taxa de 
convergência não é muito rápida, como mostra a tabela a seguir extraída do trabalho de Gröpl et al. (2001).

\begin{tabular}{|c|cccccccccccccc|}
\hline$k$ & 2 & 3 & 4 & 5 & 6 & 7 & 8 & 9 & 10 & 16 & 32 & 64 & $\cdots$ & $2^{100}$ \\
\hline$\rho_{k}$ & 2 & $\frac{5}{3}$ & $\frac{3}{2}$ & $\frac{13}{9}$ & $\frac{7}{5}$ & $\frac{15}{11}$ & $\frac{4}{3}$ & $\frac{33}{25}$ & $\frac{17}{13}$ & $\frac{5}{4}$ & $\frac{6}{5}$ & $\frac{7}{6}$ & $\cdots$ & 1,01 \\
\hline
\end{tabular}

Baseados no teorema 1.5, podemos então computar uma árvore de Steiner k-restrita ótima para uma dada instância como uma aproximação para a árvore de Steiner ótima para essa instância. No entanto, o problema kST é NP-difícil. A demonstração desse resultado é uma redução do problema da cobertura de vértices em grafos com grau máximo igual a três.

TEOREMA 1.6 O problema kST é NP-dificil para $\mathrm{k} \geqslant 4$.

Voltando ao problema ST, o algoritmo de Robins \& Zelikovsky (2000) é atualmente o algoritmo de aproximação com melhor razão de aproximação para o problema ST, estabelecida como sendo $1+(\ln 3) / 2 \leqslant 1,55$. Uma descrição detalhada desse algoritmo é uma tarefa complexa e o leitor interessado pode consultar a coletânea de Gröpl et al. (2001) ou a dissertação de Gondo (2002) que contêm a descrição, além do algoritmo de Robins \& Zelikovsky (2000), dos demais algoritmos de aproximação que já foram propostos para o problema ST. Novamente, observamos que esses algoritmos, em sua essência, fazem uso de árvores de Steiner k-restritas como aproximações para a árvore de Steiner desejada.

Além disso, mais recentemente Chlebík \& Chlebíková (2002) provaram que o limiar de aproximação do problema ST é pelo menos 1,01. Note que o intervalo entre a melhor razão de aproximação e o melhor limiar de aproximação estabelecidos para o problema ST continuam motivando os trabalhos nessa área.

\subsubsection{Problema da árvore de Steiner dos terminais folhas}

Observe que todas as folhas em uma árvore de Steiner de custo mínimo são vértices terminais, mas nem todos os vértices terminais são necessariamente folhas em uma árvore de Steiner de custo mínimo. Veja um exemplo na figura 1.1, onde as árvores 1.1(i) e 1.1(ii) são árvores de Steiner de terminais folhas e a árvore 1.1(iii) não é uma árvore de Steiner de terminais folhas.

Entretanto, para algumas aplicações que já mencionamos como construção de árvores filogenéticas em biologia, roteamento local ou global no projeto de circuitos VLSI, em transporte e telecomunicações, é necessário que todos os terminais sejam folhas na árvore de Steiner. Uma árvore de Steiner é uma árvore de Steiner de terminais folhas se o seu conjunto de vértices folhas é exatamente o conjunto de vértices terminais. Um dos problemas que tratamos neste trabalho é conhecido como problema de Steiner dos terminais folhas (terminal Steiner problem ou full Steiner problem): 
Problema TST $(G, c, R)$ : dado um grafo completo $G$, uma função custo $c: E_{G} \rightarrow \mathbb{Q} \geqslant$ satisfazendo a desigualdade triangular e um conjunto $R \subseteq V_{G}$ de terminais, encontrar uma árvore de Steiner de terminais folhas de custo mínimo em $\mathrm{G}$.

Mais uma vez, destacamos que estamos tratando aqui do problema TST métrico, isto é, G é um grafo completo e vale a desigualdade triangular para o custo das arestas de $\mathrm{G}$.

Lin \& Xue (2002) obtiveram uma $(\rho+2)$-aproximação para o problema TST, onde $\rho$ é a melhor razão de aproximação conhecida para o problema ST. Em seguida, Fuchs (2003), Lin \& Xue (2002), Chen et al. (2003) e Drake \& Hougardy (2004), obtiveram independentemente uma $2 p$-aproximação para o problema TST. Dessa forma, com o resultado de Robins \& Zelikovsky (2000), o algoritmo de Lin \& Xue (2002), é uma 3,55-aproximação para o problema TST e o algoritmo de Fuchs (2003), Chen et al. (2003) e Drake \& Hougardy (2004) é uma 3,10-aproximação para o problema TST. No capítulo 2, apresentamos dois algoritmos de aproximação para o problema TST que melhoram essa razão de aproximação. Esses algoritmos também são baseados no algoritmo de Robins \& Zelikovsky (2000), como mostraremos.

Diferentemente de uma árvore de Steiner, uma árvore de Steiner de terminais folhas não possui relação direta com uma árvore geradora de custo mínimo. De fato, como veremos, uma árvore de Steiner de terminais folhas produzida pelos algoritmos de aproximação existentes para o problema TST tem relação direta com a árvore de Steiner. Na proposição a seguir, evidenciamos a relação entre essas duas estruturas.

Proposição 1.7 Para cada C > 1, existem instâncias para as quais a razão entre o valor da solução ótima do problema TST e o valor da solução ótima do problema ST para a mesma instância é pelo menos C.

Prova. Uma instância $G, c$ e $R$ é descrita a seguir para um dado $C>1$. Fixe um conjunto de terminais $R$ com pelo menos três terminais. $O$ conjunto $V_{G}$ contém $R$ e apenas um vértice de Steiner $s$. A função custo $c$ é tal que $c\left(r r^{\prime}\right)=1$ para todo par de terminais $r, r^{\prime}$ em $R$ e $c(r s)=C$ para todo terminal $r$ em $R$ e $s$ em $V_{G} \backslash R$. A figura 1.4 mostra um exemplo de uma instância como essa.

Observe que opt $(S T(G, c, R))=|R|-1$ e que opt $(T S T(G, c, R))=C|R|$, já que $C>1$. Assim,

$$
\frac{\operatorname{opt}(T S T(G, c, R))}{\operatorname{opt}(S T(G, c, R))}=\frac{C|R|}{|R|-1} \geqslant C \text {. }
$$

Consideramos também algumas restrições sobre o problema TST. Em particular, chamamos o problema TST restrito a instâncias com arestas de custo 1 ou 2 de problema TST 1,2 . Lu et al. (2003) apresentaram duas contribuições para o problema TST $_{1,2}$ : classificaram-no mostrando que o problema é MAXSNP-difícil e propuseram uma 1,60-aproximação. No capítulo 3 , apresentamos uma 1,42-aproximação para o problema e mostramos que o problema $\mathrm{TST}_{1,2}$ é APX-completo. 


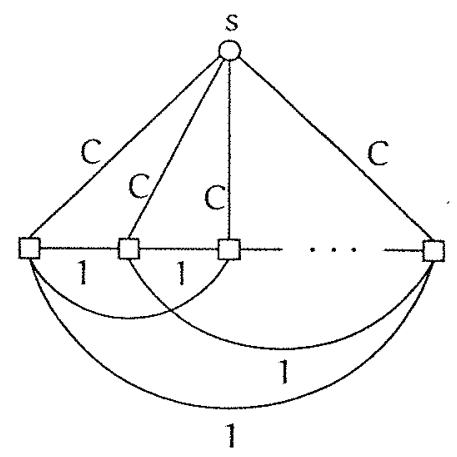

Figura 1.4: Um grafo $G$ com um único vértice de Steiner conectado a terminais por arestas de custo $C$ e os terminais conectados entre si por arestas de custo 1.

\subsubsection{Problema da árvore de Steiner com ordenação nos terminais}

No capítulo 4, consideramos ainda uma outra restrição do problema ST, impondo aos vértices terminais um ordenação obrigatória verificável numa solução. Formalmente, sejam $\mathrm{G}$ um grafo completo, $c: E_{G} \rightarrow \mathbb{Q} \geqslant$ uma função custo nas arestas de $G$ satisfazendo a desigualdade triangular, $R \subseteq V_{G}$ um conjunto de terminais e $\sigma=\left\langle\sigma_{1}, \ldots, \sigma_{|R|}\right\rangle$ uma permutação de $\{1, \ldots,|R|\}$. Seja $T$ uma árvore de Steiner para $G, c$ e $R$. Dizemos que a árvore $T$ respeita $\sigma$ se para quaisquer subíndices $i, j, k$ e $l$ tais que $1 \leqslant i<j<k<l \leqslant|R|$, então os caminhos na árvore $T$ entre os terminais $r_{\sigma_{i}}$ a $r_{\sigma_{k}}$ e entre $r_{\sigma_{j}}$ a $r_{\sigma_{l}}$ têm vértices em comum. Veja a figura 1.5 para um exemplo.

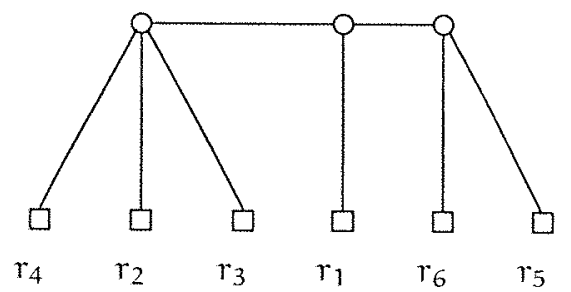

(i)

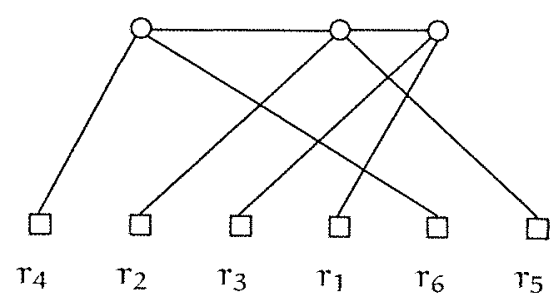

(ii)

Figura 1.5: (a) árvore que respeita a ordenação $\sigma=\langle 4,2,3,1,6,5\rangle$. (b) árvore que não respeita a ordenação $\sigma$.

Queremos resolver então o seguinte problema.

Problema STO $(G, c, R, \sigma)$ : dado um grafo completo $G$, uma função custo $c: E_{G} \rightarrow \mathbb{Q} \geqslant$ satisfazendo a desigualdade triangular, um conjunto $R \subseteq V_{G}$ de terminais e uma permutação $\sigma$ de $\{1, \ldots,|R|\}$, encontrar uma árvore de Steiner de custo mínimo em $\mathrm{G}$ que respeita $\sigma$.

Veja que se $T$ é uma árvore de Steiner para a instância $G, c$ e R que respeita a permutação $\sigma$, então $T$ obedece todas as permutações circulares de $\sigma$. A figura 1.6 mostra essa situação. 

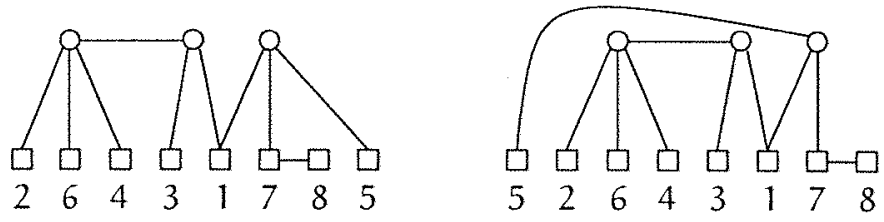

(i)

(ii)

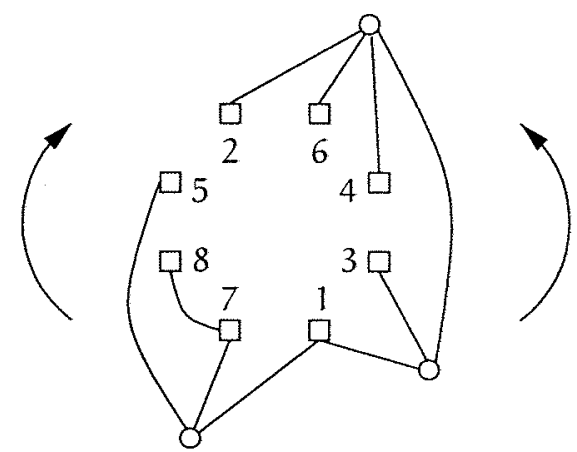

(iii)

Figura 1.6: (i) Árvore que respeita a ordenação $\sigma=\langle 2,6,4,3,1,7,8,5\rangle$. (ii) Árvore que respeita a ordenação $\sigma^{\prime}=\langle 6,4,3,1,7,8,5,2\rangle$, uma permutação circular à esquerda da permutação $\sigma$. (iii) Visualização da árvore de Steiner que respeita $\sigma$ e todas as permutações circulares de $\sigma$.

No capítulo 4 tratamos do problema STO. Apresentamos um algoritmo de aproximação que utiliza a idéia da árvore de Steiner k-restrita que respeita uma ordenação para seus terminais. Esse problema é então descrito como a seguir.

Problema $\mathrm{kSTO}(\mathrm{G}, \mathrm{c}, \mathrm{R}, \sigma, k)$ : dado um grafo completo $\mathrm{G}$, uma função custo $c: E_{G} \rightarrow \mathbb{Q} \geqslant$, um conjunto $R \subseteq V_{G}$ de terminais, uma permutação $\sigma$ de $\{1, \ldots,|R|\}$ e um inteiro positivo $k$, encontrar uma árvore de Steiner k-restrita de custo mínimo em $\mathrm{G}$ que respeita $\sigma$.

Propomos um algoritmo eficiente para solucionar o problema kSTO, mostrando que esse problema está na classe de complexidade PO. Além disso, mostramos que esse algoritmo proposto é um esquema de aproximação polinomial para o problema STO, mostrando então que esse problema está na classe de complexidade PTAS. 


\section{Capítulo 2}

\section{Problema TST}

No problema da árvore de Steiner de terminais folhas queremos encontrar em um dado grafo, com custos nas arestas e com um subconjunto de vértices chamados terminais, um subgrafo de custo mínimo que conecte os terminais e que esses terminais tenham grau 1. Esse subgrafo é uma árvore, chamada árvore de Steiner de terminais folhas, onde todos os terminais são folhas. Aplicações como construção de árvores filogenéticas, roteamento local ou global no projeto de circuitos VLSI, em transporte e telecomunicações têm sido citadas na literatura (Lin \& Xue, 2002; Fuchs, 2003; Chen et al., 2003; Drake \& Hougardy, 2004).

São recentes os resultados obtidos para o problema TST. Lin \& Xue (2002) propuseram um algoritmo com razão de aproximação $\rho+2$ para o problema TST, onde $\rho$ é a razão de aproximação do melhor algoritmo de aproximação existente para o problema de Steiner em grafos. Em seguida, Fuchs (2003), Chen et al. (2003) e Drake \& Hougardy (2004), obtiveram independentemente um algoritmo com razão de aproximação $2 \rho$ para o problema TST. Atualmente, o algoritmo proposto por Robins \& Zelikovsky $(2000)$ estabelece $\rho=1+(\ln 3) / 2 \leqslant$ 1,55. Dessa forma, temos uma 3,55-aproximação para o problema TST no trabalho de Lin \& Xue (2002) e uma 3,10-aproximação nos trabalhos de Fuchs (2003), Chen et al. (2003) e Drake \& Hougardy (2004).

Trabalhamos em conjunto com o professor José Coelho de Pina Júnior e propomos um algoritmo de aproximação com razão de aproximação 2,76 para o problema TST que também utiliza o algoritmo de Robins \& Zelikovsky (2000) como uma subrotina. Durante a formalização das idéias envolvidas nessa aproximação, juntamente com sugestōes de um revisor de um trabalho nosso submetido a uma conferência, acabamos por descobrir um outro algoritmo que melhora essa razão de aproximação para 2,52. Mostramos também que o problema TST é APXcompleto.

Este capítulo está organizado da seguinte forma. Apresentamos o algoritmo de Drake \& Hougardy (2004) na seção 2.1. Na seção 2.2 apresentamos a 2,76-aproximação para o problema TST e a 2,52-aproximação na seção 2.3. E, finalmente, apresentamos uma prova que o problema TST é APX-completo na seção 2.4. 


\subsection{Uma 3,10-aproximação para o problema TST}

Sejam G, c e R uma instância do problema TST tal que R contém pelo menos três terminais. Como uma árvore de Steiner de terminais folhas não contém arestas conectando terminais, podemos considerar que para cada par de vértices $q, r$ em $R$, o custo da aresta qr em $E_{G}$ é $\min _{v \in V_{G} \backslash R}(c(q v)+c(v r))$. Em particular, isso implica que podemos considerar que toda árvore de Steiner para uma instância $G$, c e R não contém arestas contectando vértices terminais. Como os resultados de Fuchs (2003), Chen et al. (2003) e de Drake \& Hougardy (2004) são equivalentes em termos da razão de aproximação obtida, optamos aqui por apresentar apenas um desses trabalhos. A seguir, descrevemos o algoritmo de Drake \& Hougardy (2004) e apresentamos uma demonstração de que o algoritmo é uma $2 \rho$-aproximação para o problema TST, onde $\rho$ é a melhor razão de aproximação para o problema ST.

O algoritmo faz uso da operação de substituição de uma estrela, que passamos a descrever. Suponha que $r$ em R é um vértice de grau $j>1$ em uma árvore de Steiner T obtida por uma $\rho$-aproximação sobre a instância $G, c$ e R. O vértice $r$ é chamado uma estrela (de Steiner) de $T$. Sejam $v_{1}, v_{2}, \ldots, v_{j}$ os vértices adjacentes a $r$ em $T$ e observe que $v_{1}, v_{2}, \ldots, v_{j}$ são vértices de Steiner, isto é, $\left\{v_{1}, v_{2}, \ldots, v_{j}\right\} \subseteq V_{G} \backslash R$. Suponha, sem perda de generalidade, que $c\left(r v_{1}\right) \leqslant$ $c\left(r v_{\mathfrak{i}}\right)$, para $\mathfrak{i}=1,2, \ldots, j$. A operação de substituição da estrela reduz o grau de um vértice terminal $r$ em $R$ para grau 1 através da troca das arestas $r v_{2}, r v_{3}, \ldots, r v_{j}$ de $E_{T}$ pelas arestas $v_{1} v_{2}, v_{1} v_{3}, \ldots, v_{1} v_{j}$ de $E_{G}$, como mostra a figura 2.1. Observe que a operação de substituição de uma estrela transforma um vértice interno $r$ de $T$ em uma folha e os graus dos outros vértices são mantidos inalterados. Observe ainda que um par de operações como essa para terminais diferentes envolve conjuntos de arestas diferentes.

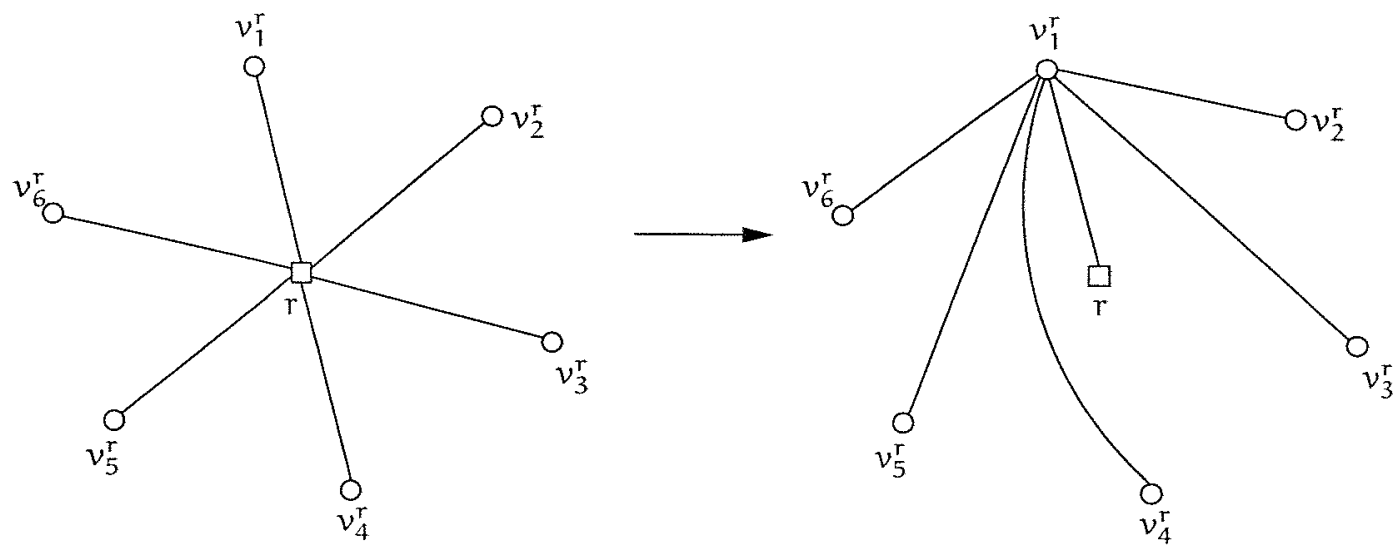

Figura 2.1: A operação de substituição da estrela $r$.

O ALGORITMO ESTRELA de Drake \& Hougardy (2004) recebe uma instância G, c e R do problema TST, constrói uma árvore de Steiner para essa instância usando uma aproximação para o problema ST, substitui as estrelas de Steiner nessa árvore e devolve essa estrutura modificada. A estrutura devolvida é uma árvore de Steiner de terminais folhas para a instância $G, c$ e $R$, e como veremos no teorema 2.1, o custo dessa árvore dista no máximo 2 vezes do custo da árvore de Steiner de terminais folhas para essa instância. 
ALGORITMO ESTRELA: recebe um grafo completo $G$ a menos de arestas entre os terminais, uma função custo $c: E_{G} \rightarrow \mathbb{Q} \geqslant$ satisfazendo a desigualdade triangular e um conjunto $R \subseteq V_{G}$ de terminais, e devolve uma árvore de Steiner de terminais folhas.

1: Compute uma árvore de Steiner $T \rho$-aproximada para a instância ( $G, c, R$ ).

2: Para cada $r$ em $R$ tal que $r$ não é folha em $T$, faça uma substituição da estrela $r$.

3: Devolva T e pare.

TEOREMA 2.1 (Drake \& Hougardy, 2004) O ALGORITMO ESTRELA é uma $2 \rho$-aproximação para o problema TST, onde $\rho$ é a melhor razão de aproximação conhecida para o problema ST.

Prova. Chame de $T^{\prime}$ uma árvore obtida pela $\rho$-aproximação, após a execução da linha 1 do ALGORITMO ESTRELA sobre uma instância G, c e R do problema TST. Suponha que $R^{\star} \subseteq R$ é o conjunto contendo as estrelas em $T^{\prime}$ e chame de $T$ uma solução viável obtida pelo ALGORITMO ESTRELA para a instância $G, c$ e R, após o algoritmo ter realizado uma seqüência de operações de substituição de estrelas sobre $R^{\star}$ em $T^{\prime}$.

Para cada estrela r em $R^{\star}$ de $T^{\prime}$, denote por $k_{r}$ o grau de $r$ em $T^{\prime}$, onde $k_{r}>1$, e por $v_{1}^{r}, \ldots, v_{k}^{r}$ seus vizinhos em $T^{\prime}$. Suponha ainda, sem perda de generalidade, que $c\left(r v_{1}^{r}\right) \leqslant c\left(r v_{j}^{r}\right)$ para todo $j, 1 \leqslant j \leqslant k_{r}$. Então, o custo da árvore T devolvida pelo ALGORITMO ESTRELA é dado por

$$
c(T)=\sum_{r \in R^{\star}}\left(c\left(r v_{1}^{r}\right)+\sum_{j=2}^{k_{r}} c\left(v_{1}^{r} v_{j}^{r}\right)\right)+c(X)
$$

onde $X$ é um conjunto de arestas de $T^{\prime}$, e de T, que não fazem parte de estrela alguma. Assim,

$$
\begin{aligned}
c(T) & =\sum_{r \in R^{\star}}\left(c\left(r v_{1}^{r}\right)+\sum_{j=2}^{k_{r}} c\left(v_{1}^{r} v_{j}^{r}\right)\right)+c(X) \\
& \leqslant \sum_{r \in R^{\star}}\left(c\left(r v_{1}^{r}\right)+\sum_{j=2}^{k_{r}}\left(c\left(v_{1}^{r} r\right)+c\left(r v_{j}^{r}\right)\right)\right)+c(X) \\
& \leqslant \sum_{r \in R^{\star}}\left(c\left(r v_{1}^{r}\right)+\sum_{j=2}^{k_{r}} 2 c\left(r v_{j}^{r}\right)\right)+c(X) \\
& \leqslant 2\left(\sum_{r \in R^{\star}}\left(c\left(r v_{1}^{r}\right)+\sum_{j=2}^{k_{r}} c\left(r v_{j}^{r}\right)\right)+c(X)\right) \\
& =2 c\left(T^{\prime}\right) \\
& \leqslant 2(\rho \operatorname{opt}(\operatorname{ST}(G, c, R))) \\
& \leqslant 2 p \operatorname{opt}(\operatorname{TST}(G, C, R))
\end{aligned}
$$

onde a desigualdade (2.1) vale pela desigualdade triangular, a desigualdade (2.2) vale pela suposição que $c\left(r v_{1}^{r}\right) \leqslant c\left(r v_{j}^{r}\right)$ para todo $1 \leqslant j \leqslant k_{r}$ e toda estrela $r$ em $R^{\star}$, a desigualdade (2.3) 
vale devido ao uso de uma $\rho$-aproximação para obter $T^{\prime}$ e finalmente a desigualdade (2.4) vale porque o custo de uma árvore de Steiner ótima é um limitante inferior para o custo de uma árvore de Steiner de terminais folhas ótima.

\subsection{Uma 2,76-aproximação para o problema TST}

Propomos a seguir um algoritmo de aproximação polinomial para o problema TST que constrói duas árvores de Steiner de terminais folhas para uma instância G, c e R do problema, com a garantia que uma delas é uma boa aproximação para o problema TST. Esse algoritmo melhora a razão de aproximação $2 \rho$ apresentada na seção anterior.

Sejam G, c e R uma instância para o problema TST, com $|R| \geqslant 3$. Da mesma forma como antes, como uma árvore de Steiner de terminais folhas não contém arestas conectando terminais, podemos considerar que para cada par de vértices $q, r$ em $R$, o custo da aresta qr em $E_{G}$ é $\min _{v \in V_{G} \backslash R}(c(q v)+c(v r))$. Em particular, isso implica que podemos considerar que toda árvore de Steiner para uma instância $G, c$ e R não contém arestas contectando vértices terminais.

Para cada $r$ em $R$, denotamos por $e_{r}$ uma aresta de menor custo conectanto $r$ a $V_{G} \backslash R$ e denotamos por $F_{R}$ um conjunto $\left\{e_{r}: r \in R\right\}$. Apresentamos a seguir um algoritmo de aproximação que recebe $G, c$ e $R$ e constrói duas árvores de Steiner de terminais folhas $T_{1}$ e $T_{2}$ tais que

$$
c\left(T_{1}\right) \leqslant \alpha_{2} \operatorname{opt}(\operatorname{TST}(G, c, R))
$$

e se o valor $c\left(F_{R}\right)$ é 'pequeno' comparado a $\operatorname{opt}(T S T(G, c, R))$ então

$$
c\left(T_{1}\right) \leqslant \alpha_{1} \operatorname{opt}(\operatorname{TST}(G, c, R))
$$

caso contrário

$$
c\left(T_{2}\right) \leqslant \alpha_{1} \operatorname{opt}(\operatorname{TST}(G, c, R)) .
$$

O algoritmo devolve então a árvore construída de menor custo e melhora a razão de aproximação $2 \rho$ apresentada na seção anterior, como mostraremos.

TEOREMA 2.2 Existe um algoritmo de aproximação de tempo polinomial para o problema TST com razāo de aproximaçāo $\alpha=\min \left\{\alpha_{1}, \alpha_{2}\right\}$ onde

$$
\alpha_{1}=1+\frac{10 \rho(\rho-1)}{7 \rho-6}, \quad \alpha_{2}=4 \rho-3
$$

supondo que um algoritmo de aproximação de tempo polinomial obtém uma razão de aproximação $\rho \leqslant 2$ para o problema ST.

Como o melhor valor conhecido para $\rho$ é ligeiramente menor que 1,55 o teorema 2.2 faz $\alpha=\alpha_{1}$ e implica o seguinte corolário.

COROLÁRIO 2.3 Existe uma 2,76-aproximação para o problema TST. 


\subsubsection{Algoritmo Árvore}

O objetivo do ALGORITMO ÁRVORE 1 é construir uma árvore de Steiner de terminais folhas, a árvore $T_{1}$ citada na seção anterior, na tentativa de que essa árvore seja uma boa aproximação para o problema TST. A idéia é que o algoritmo inicialmente modifique os custos das arestas que incidem em vértices terminais da instância de entrada. Em seguida, uma árvore de Steiner é construída para essa instância modificada. Finalmente, transformações semelhantes à substituição da estrela descrita na seção 2.1 são realizadas sobre essa árvore de Steiner de tal forma que o algoritmo obtém uma árvore de Steiner de terminais folhas. Mostramos então que essa árvore resultante é, sob certas hipóteses, uma boa aproximação para uma árvore de Steiner de terminais folhas de custo ótimo.

A proposição a seguir apresenta uma situação em que opt $(\operatorname{TST}(G, c, R))=\operatorname{opt}(S T(G, c, R))$.

PROPOSIÇÃo 2.4 Sejam G, c e R uma instância do problema TST. Suponha que para cada aresta $e_{\mathrm{r}}=\mathrm{ru}, \mathrm{r}$ em $\mathrm{R}$, e para cada vértice $w$ em $\mathrm{V}_{\mathrm{G}}-\mathrm{r}$ temos que

$$
c(u w) \leqslant c(r w)-\frac{c(r u)}{2} .
$$

Então, dada uma árvore de Steiner S para a instância $G, c e$, podemos obter em tempo polinomial uma árvore de Steiner de terminais folhas $\mathrm{T}$ tal que $\mathrm{c}(\mathrm{T}) \leqslant \mathrm{c}(\mathrm{S})$.

PROVA. Se cada vértice em $R$ é uma folha na árvore de Steiner $S$ então podemos fazer $T:=S$ e terminamos a demonstração. Dessa forma, podemos assumir que existe um vértice $r$ em $R$ tal que seu conjunto de vizinhos $W$ na árvore $S$ tem mais que um vértice. Sejam $u$ um vértice tal que $e_{r}=r u, X:=\{r w: W \in W\}$ um conjunto de arestas que conectam $r$ aos vértices de $W$ e $Y:=\{u w: w \in W\}$ um conjunto de arestas que conectam o vértice $u$ aos vértices de $W$. Veja a figura 2.2 para uma ilustração dessas definições.

Finalmente, seja $S^{\prime}$ a árvore de Steiner induzida pelas arestas em $\left(E_{S} \backslash X\right) \cup Y \cup\{r u\}$. Temos então que

$$
\begin{aligned}
c\left(S^{\prime}\right) & =c\left(E_{S}\right)-c(X)+c(Y)+c(r u) \\
& \leqslant c\left(E_{S}\right)-c(X)+c(X)-|W| \frac{c(r u)}{2}+c(r u) \\
& =c\left(E_{S}\right)+\left(1-\frac{|W|}{2}\right) c(r u) \\
& \leqslant c\left(E_{S}\right) \\
& =c(S)
\end{aligned}
$$

onde vale a desigualdade (2.5) porque

$$
c(u w) \leqslant c(r w)-\frac{c(r u)}{2},
$$

para cada $w$ em $W$. 


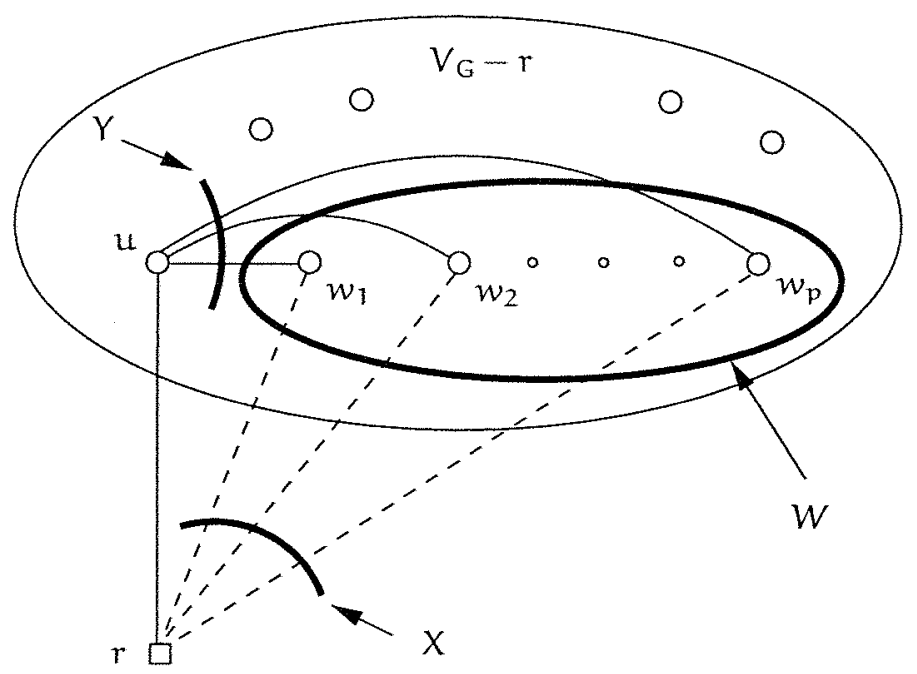

Figura 2.2: Ilustração para a proposição 2.4. Arestas pontilhadas são removidas e arestas sólidas são incluídas. $O$ vértice $u$ pode estar contido no conjunto $W$.

Nesse processo, obtemos uma árvore de Steiner onde r é uma folha e todos os terminais que são folhas em $S$ permanecem folhas em $S^{\prime}$. Assim, repetindo esse processo, finalizamos com uma árvore de Steiner de terminais folhas $T$ satisfazendo a proposição.

O ALGORITMO ÁRVORE 1 recebe uma instância G, c e R do problema TST e modifica inicialmente os custos das arestas de $G$ tal que para cada terminal $r$ em $R$ é adicionado ao custo de uma aresta que incide em $R$ o valor de três vezes o custo de uma aresta $e_{r}$ de menor custo que incide em $r$. Veja a figura 2.3 para uma ilustração.

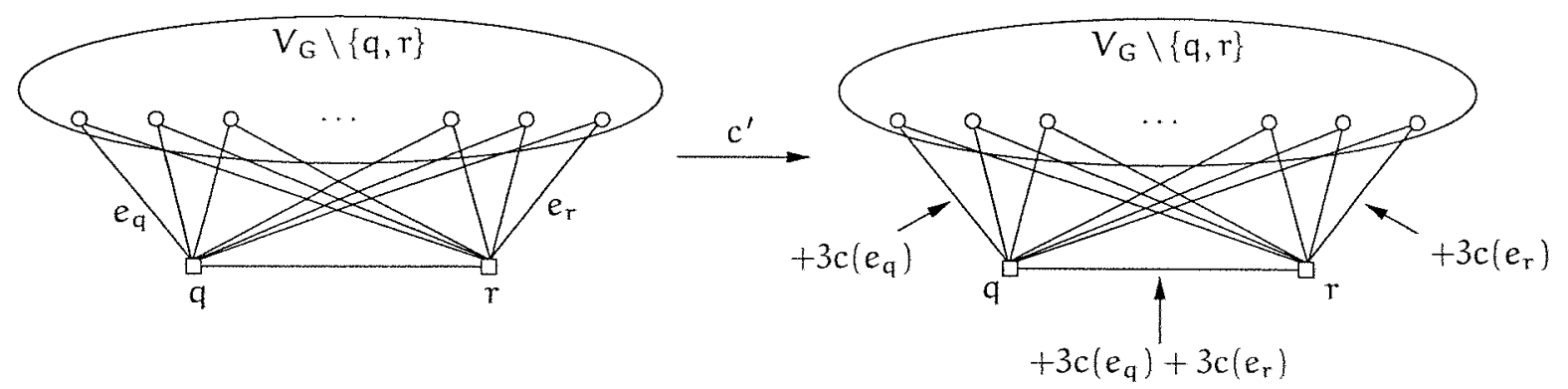

Figura 2.3: A função $c^{\prime}$ sobre $G$ com dois terminais.

Essa nova função custo sobre as arestas de $G$ é então chamada de $c^{\prime}$ e uma árvore de Steiner $S$ é construída para a instância $G, c^{\prime}$ e $R$. Como a função $c^{\prime}$ satisfaz as hipóteses da proposição 2.4, então podemos construir uma árvore de Steiner de terminais folhas $T$ a partir de $S$ com custo no máximo o custo de $S$. Observe que a função $c^{\prime}$ assim construída e o ALGORITMO ÁRVORE 1 fazem com que a árvore de Steiner de terminais folhas $T$ escolha a aresta $e_{\mathrm{T}}$ para conectar $r$ em $T$. A árvore T é então devolvida pelo ALGORITMO ÁRVORE ${ }_{1}$. 
ALGORITMO ÁRVORE 1 : recebe um grafo completo $G$, uma função custo $c: E_{G} \rightarrow \mathbb{Q} \geqslant$ satisfazendo a desigualdade triangular e um conjunto $R \subseteq V_{G}$ de terminais, e devolve uma árvore de Steiner de terminais folhas.

1: Seja $c^{\prime}: E_{G} \rightarrow \mathbb{Q} \geqslant$ definida como

$$
c^{\prime}(e):= \begin{cases}c(e)+3 c\left(e_{q}\right)+3 c\left(e_{r}\right), & \text { se } e \cap R=\{q, r\} \\ c(e)+3 c\left(e_{r}\right), & \text { se } e \cap R=\{r\} \\ c(e), & \text { se } e \cap R=\emptyset .\end{cases}
$$

2: Seja $S$ uma árvore de Steiner devolvida por uma $\rho$-aproximação para o problema ST com instância $G, c^{\prime}$ e R.

3: Construa uma árvore de Steiner de terminais folhas T a partir de $S$ usando a proposição 2.4.

4: Devolva T e pare.

TEOREMA 2.5 Sejam $\mathrm{G}$, c e R uma instância para o problema $\mathrm{TST}, \mathrm{F}_{\mathrm{R}}:=\left\{e_{\mathrm{r}}: r \in \mathrm{R}\right\}$ um conjunto de arestas de menor custo que atingem os terminais e $\mathrm{T}$ a árvore de Steiner de terminais folhas devolvida pelo ALGORITMO ÁRVORE 1 . Se $\beta$ é um número tal que

$$
\rho \operatorname{opt}(\operatorname{TST}(G, c, R))+3(\rho-1) c\left(F_{R}\right) \leqslant \beta \operatorname{opt}(T S T(G, c, R))
$$

então

$$
c(T) \leqslant \beta \operatorname{opt}(\operatorname{TST}(G, c, R))
$$

PROVA. Sejam c' e S a função custo e a árvore de Steiner computadas pelo algoritmo, respectivamente. Como $c^{\prime}$ satisfaz a hipótese da proposição 2.4 , então $c^{\prime}(T) \leqslant c^{\prime}(S)$. Além disso, para cada $r$ em $R$ a aresta incidente a $r$ em $T$ é $e_{r}$. Portanto,

$$
\begin{aligned}
c(T) & =c^{\prime}(T)-3 c\left(F_{R}\right) \\
& \leqslant c^{\prime}(S)-3 c\left(F_{R}\right) \\
& \leqslant \rho \operatorname{opt}\left(S T\left(G, c^{\prime}, R\right)\right)-3 c\left(F_{R}\right) \\
& \leqslant \rho\left(\operatorname{opt}(T S T(G, c, R))+3 c\left(F_{R}\right)\right)-3 c\left(F_{R}\right) \\
& =\rho \operatorname{opt}(T S T(G, c, R))+3(\rho-1) c\left(F_{R}\right) \\
& \leqslant \beta \operatorname{opt}(T S T(G, c, R)),
\end{aligned}
$$

onde a desigualdade (2.6) vale porque se $T^{*}$ é uma árvore de Steiner de terminais folhas tal que $c\left(T^{*}\right)=\operatorname{opt}(T S T(G, c, R))$ então

$$
\operatorname{opt}\left(S T\left(G, c^{\prime}, R\right)\right) \leqslant c^{\prime}\left(T^{*}\right)=c\left(T^{*}\right)+3 c\left(F_{R}\right)=\operatorname{opt}(T S T(G, c, R))+3 c\left(F_{R}\right)
$$

Dessa forma, o ALGORITMO ÁRVORE 1 constrói uma árvore de Steiner de terminais folhas que, sob certas condições, é uma boa aproximação para uma árvore de Steiner de terminais folhas ótima. Essas condições ficarão mais claras na seção 2.2.3. 


\subsubsection{Algoritmo Árvore 2}

O ALGORITMO ÁRVORE 2 constrói uma segunda árvore, a árvore $T_{2}$ já mencionada anteriormente, que pode ser uma boa aproximação para o problema TST. O algoritmo conecta de alguma forma os vértices terminais a vértices de Steiner escolhidos na instância de entrada. Em seguida, constrói uma árvore de Steiner para uma instância onde esses vértices de Steiner são tratados como vértices terminais. No final, o ALGORITMO ÁRVORE 2 simplesmente conecta essa árvore de Steiner assim obtida com os vértices terminais da instância original. Descrevemos mais formalmente esse algoritmo abaixo.

Seja $G$ um grafo completo com uma função custo $c$ nas suas arestas satisfazendo a desigualdade triangular e seja $R$ um subconjunto de vértices de $G$. Um desmembramento de uma árvore $T$ no grafo $G$ é uma coleção de cardinalidade máxima de subárvores aresta-disjuntas, cada árvore contendo pelo menos dois vértices de $R$ e todo vértice de $R$ pertencendo a alguma dessas subárvores. Note que, como cada folha de $T$ tem grau 1 , tal folha ocorre em exatamente uma subárvore de um desmembramento. A figura 2.4 mostra desmembramentos de duas árvores.

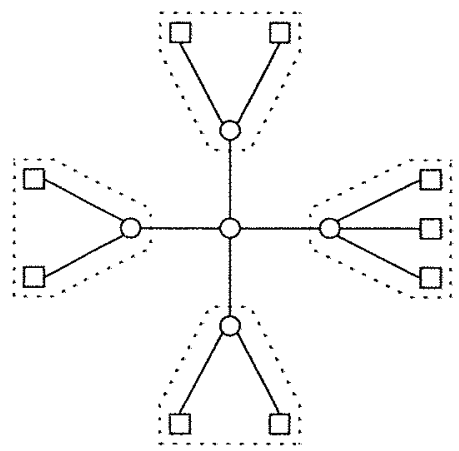

(a)

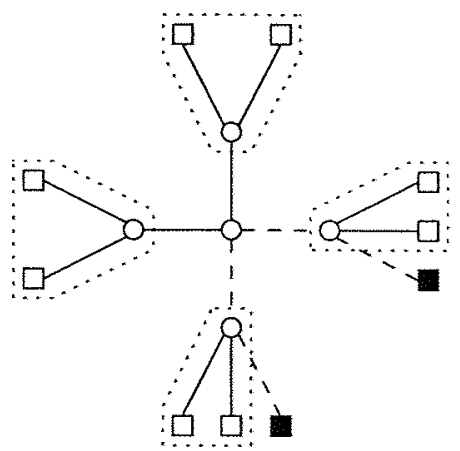

(b)

Figura 2.4: Desmembramentos para duas árvores. O caminho tracejado entre os terminais preenchidos na árvore (b) é uma subárvore do desmembramento dessa árvore.

LEMA 2.6 Se Té uma árvore com pelo menos duas folhas, então um desmembramento de T tem cardinalidade $[|R| / 2\rfloor$, onde Ré o conjunto de folhas de $\mathrm{T}$.

PROVA. É fácil ver que a cardinalidade de um desmembramento de $T$ não pode ser maior que $[|R| / 2]$. Dessa forma, mostramos por indução em $|R|$ que $T$ tem um desmembramento com essa cardinalidade.

Se $|R| \leqslant 3$, então $T$ é um desmembramento de $T$. Assim, podemos supor que $|R|>3$. Considere $T$ como uma árvore com raiz, escolhendo como raiz qualquer vértice de $V_{G} \backslash R$ em $T$. Seja $v$ um vértice em $T$ que tem como descendentes imediatos pelo menos 2 folhas, digamos $r$ e $s$, e nenhum vértice de grau maior que 2. Seja $T^{\prime}$ uma subárvore de $T \operatorname{com} R^{\prime}:=R \backslash\{r, s\}$ como conjunto de suas folhas. Por indução, $T^{\prime}$ tem um desmembramento de cardinalidade 
$\left\lfloor R^{\prime} \mid / 2\right\rfloor$. Este desmembramento juntamente com um caminho em $T$ conectando r e s compõem um desmembramento de $T$ com $[|R| / 2\rfloor$ elementos.

Sejam G, c e R uma instância do problema TST e seja T uma árvore de Steiner de terminais folhas. Como toda aresta de $T$ ocorre em no máximo uma subárvore de um desmembramento $S$ de $T$, sabemos que $c(S) \leqslant c(T)$, onde $c(S):=\sum_{J \in S} c(J)$ é o custo de um desmembramento. Um desmembramento de $(G, c, R)$ é um desmembramento de alguma árvore de Steiner de terminais folhas.

TEOREMA 2.7 Um desmembramento de custo mínimo de (G, $c, R)$ pode ser encontrado em tempo polinomial.

Prova. Inicialmente, considere $|R|$ par. Seja $H$ um grafo completo tal que $V_{H}:=R$ e seja $f: E_{H} \rightarrow \mathbb{Q} \geqslant$ tal que $f(q r)=\min _{v \in V_{G} \backslash R}\{c(q v)+c(\nu r)\}$. Segue do lema 2.6 que qualquer desmembramento $\mathcal{S}$ de $\mathrm{G}$ corresponde a um emparelhamento perfeito $M$ em $\mathrm{H}$, e vice-versa. Além disso, pela definição de $f, f(M) \leqslant c(S)$. Considerando a função $f$, um emparelhamento perfeito $M$ de custo mínimo em $H$ induz um desmembramento $S$ em $G$ tal que $f(M)=c(S)$. Como $f(M)$ é mínimo, $c(\delta)$ também é mínimo e $S$ é então um desmembramento de custo mínimo. Observando que um emparelhamento perfeito de custo mínimo pode ser encontrado em tempo polinomial (Schrijver, 2003), finalizamos o caso em que $|R|$ é par.

Quando $|R|$ é ímpar, procedemos da seguinte forma. Considere $R^{\prime} \subseteq R$ tal que $\left|R^{\prime}\right|=3$. Como $\left|R \backslash R^{\prime}\right|$ é par, podemos resolver o problema para a instância $\left(G-R^{\prime}, c, R \backslash R^{\prime}\right)$. Esta solução, em conjunto com uma árvore de custo mínimo que conecta os vértices em $R^{\prime}$, é uma candidata a ser um desmembramento de custo mínimo de $(G, c, R)$. Tomando o mínimo sobre os custos de todas as candidatas, uma para cada tripla $R^{\prime}$ de vértices de $R$, um desmembramento de custo mínimo para $(G, c, R)$ é encontrado.

O lema a seguir mostra que podemos construir eficientemente um desmembramento de custo mínimo com poucos vértices de Steiner.

LEMA 2.8 Dado um desmembramento de custo mínimo $S$ de $(G, c, R)$, podemos encontrar em tempo polinomial um desmembramento de custo mínimo $S^{\prime}$ de $(G, c, R)$ tal que cada árvore em S'é uma estrela com centro em $V_{\mathrm{G}} \backslash R$.

Prova. Podemos assumir que não existem arestas conectando pares de vértices em $R$. Assim, cada árvore em $\mathcal{S}$ tem pelo menos um vértice de $V_{\mathrm{G}} \backslash R$. Suponha que existe uma árvore $J$ em $S$ que não é uma estrela. Seja $J^{\prime}$ uma árvore com centro em $V_{J} \backslash R$ e com folhas em $V_{J} \cap R$. Pela desigualdade triangular $c\left(J^{\prime}\right) \leqslant c(J)$. Então, $\mathcal{S}^{\prime}:=\mathcal{S} \backslash\{J\} \cup\left\{J^{\prime}\right\}$ é um desmembramento de custo mínimo contendo uma estrela a mais que $\mathcal{S}$. Repetindo esse processo, finalizamos com um desmembramento satisfazendo o lema. 
LEMA 2.9 Sejam G, c e R uma instância do problema TST. Dado um desmembramento S de (G, c, R) composto por estrelas, com $R^{\prime}$ como conjunto de centros, e uma árvore de Steiner $S$ de $\left(G-R, c, R^{\prime}\right)$, podemos encontrar em tempo polinomial uma árvore de Steiner de terminais folhas $T$ de $(G, c, R)$ tal que

$$
c(T)=c(S)+c(S)
$$

PROVA. Defina T adicionando à arvore $S$ as arestas do desmembramento $S$. Veja a figura 2.5.

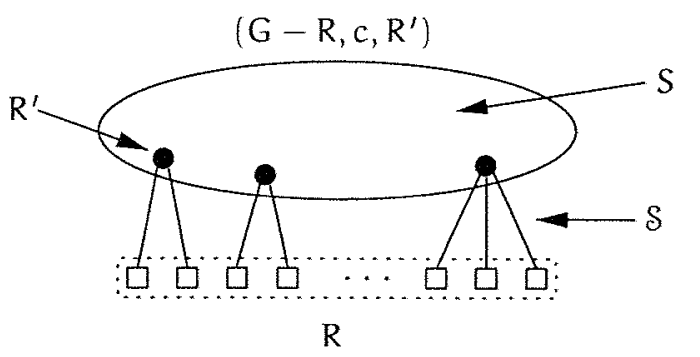

Figura 2.5: Uma ilustração para o lema 2.9.

O ALGORITMO ÁRVORE, 2 recebe uma instância G, c e R do problema TST e constrói inicialmente um desmembramento de custo mínimo $\delta$ composto de estrelas para essa instância. Em seguida, o algoritmo constrói uma árvore de Steiner $S$ para uma instância reduzida, removendo de $G$ os vértices em $R$ e chamando os centros das estrelas do desmembramento $\mathcal{S}$ de terminais nessa instância reduzida. Por fim, o algoritmo conecta a árvore de Steiner $S$ ao desmembramento $\delta$ e devolve essa árvore resultante.

ALGORITMO ÁRVORE 2 : recebe um grafo completo $G$, uma função custo $c: E_{G} \rightarrow \mathbb{Q} \geqslant$ satisfazendo a desigualdade triangular e um conjunto $R \subseteq V_{G}$ de terminais, e devolve uma árvore de Steiner de terminais folhas.

1: Seja $S$ um desmembramento de custo mínimo de $(G, c, R)$ composto de estrelas, construído conforme o teorema 2.7 e o lema 2.8 .

2: Seja $R^{\prime}$ o conjunto de centros das estrelas em $\delta$.

3: Seja $S$ a árvore de Steiner devolvida por uma $\rho$-aproximação para o problema $S T(G-$ $\left.R, c, R^{\prime}\right)$.

4: Construa uma árvore de Steiner de terminais folhas $T$ a partir de $S$ e $S$ usando o lema 2.9.

5: Devolva T e pare.

O lema a seguir estabelece uma relação entre $S, S$ e T devolvidos pelo ALGORITMO ÁRVORE 2 e arestas de menor custo que incidem nos terminais.

LEMA 2.10 Sejam G, c e R uma instância para o problema $T S T, F_{R}:=\left\{e_{r}: r \in R\right\}$ um conjunto de arestas de menor custo que atingem os terminais e $\&$ um desmembramento de custo mínimo de $(G, c, R)$ composto de estrelas. Se $R^{\prime}$ é o conjunto de centros de estrelas em $\mathcal{S}$, então

$$
\operatorname{opt}\left(S T\left(G-R, c, R^{\prime}\right)\right) \leqslant \operatorname{opt}(T S T(G, c, R))+\frac{c(S)}{2}-\frac{c\left(F_{R}\right)}{2} .
$$


Prova. Seja $T^{*}$ uma árvore de Steiner de terminais folhas tal que $c\left(T^{*}\right)=\operatorname{opt}(\operatorname{TST}(G, c, R)) \mathrm{e}$ para cada $r$ em $R$ seja $f_{r}=r w_{r}$ uma aresta em $T^{*}$ incidente em $r$.

Sejam $W:=\left\{w_{r}: r \in R\right\}$ um conjunto de vértices de Steiner ligados aos terminais de $R$ em $T^{*}$ e $F:=\left\{f_{r}: r \in R\right\}$ um conjunto das arestas incidentes nos terminais de $R$ em $T^{*}$. Para cada $r^{\prime}$ em $R^{\prime}$, denotamos por $d_{r^{\prime}}$ uma aresta de custo mínimo conectando $r^{\prime}$ a um vértice em $W$. Denote ainda por $D:=\left\{d_{r^{\prime}}: r^{\prime} \in R^{\prime}\right\}$ um conjunto de arestas assim definidas.

Para provar o lema, mostramos que um subgrafo $S$ induzido pelas arestas em $\left(E_{T^{*}} \backslash F\right) \cup D$ contém uma árvore de Steiner para $\left(G-R, c, R^{\prime}\right)$ e que

$$
c(S) \leqslant \operatorname{opt}(\operatorname{TST}(G, c, R))+\frac{c(S)}{2}-\frac{c\left(F_{R}\right)}{2} .
$$

Sabemos que $S$ conecta os vértices em $R^{\prime}$. Assim, podemos considerar que $S$ é uma árvore de Steiner para a instância $\left(G-R, c, R^{\prime}\right)$. Temos então que

$$
\begin{aligned}
c(S) & =c\left(T^{*}\right)-c(F)+c(D) \\
& =\operatorname{opt}(\operatorname{TST}(G, c, R))-c(F)+c(D) \\
& \leqslant \operatorname{opt}(\operatorname{TST}(G, c, R))-c(F)+\frac{c(S)+c(F)}{2} \\
& =\operatorname{opt}(\operatorname{TST}(G, c, R))+\frac{c(S)}{2}-\frac{c(F)}{2} \\
& \leqslant \operatorname{opt}(\operatorname{TST}(G, c, R))+\frac{c(S)}{2}-\frac{c\left(F_{R}\right)}{2}
\end{aligned}
$$

A desigualdade (2.8) vale pela definição de $F$ e $F_{R}$. A justificativa para a passagem onde vale a desigualdade (2.7) será feita a seguir. Para cada $r$ em $R$, seja $g_{r}$ a aresta incidente a $r$ na estrela de $\mathcal{S}$ que contém $r$ e seja $h_{r}$ a aresta que compõe um triângulo com as arestas $g_{r}$ e $f_{r}$. Veja a figura 2.6 para uma ilustração.

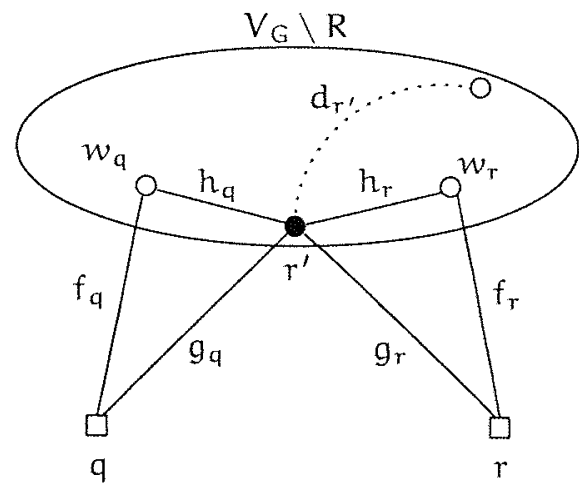

Figura 2.6: Arestas definidas a partir de $\delta$ e T*. 
Existem pelo menos dois vértices $q$ e $r$ em $R$ tais que $g_{q}=q r^{\prime}$ e $g_{r}=r r^{\prime}$. Portanto,

$$
\begin{aligned}
c\left(d_{r^{\prime}}\right) & \leqslant \frac{c\left(h_{q}\right)+c\left(h_{r}\right)}{2} \\
& \leqslant \frac{c\left(g_{q}\right)+c\left(f_{q}\right)+c\left(g_{r}\right)+c\left(f_{r}\right)}{2},
\end{aligned}
$$

onde a primeira desigualdade vale devido à escolha de $d_{r^{\prime}}$ e a segunda segue da desigualdade triangular. Portanto, se $H$ denota o conjunto $\left\{h_{r}: r \in R\right\}$, pela desigualdade (2.9) temos que

$$
c(D) \leqslant \frac{c(H)}{2} \leqslant \frac{c(S)+c(F)}{2}
$$

e isso completa a prova do lema.

TEOREMA 2.11 Sejam $G, c$ e $R$ uma instância do problema TST, $F_{R}:=\left\{e_{r}: r \in R\right\}$ um conjunto de arestas de menor custo que atingem os terminais $e \mathrm{~T}$ a árvore de Steiner de terminais folhas devolvida pelo ALGORITMO ÁRVORE2. Se ß é tum número tal que

$$
\left(\frac{3}{2} \rho+1\right) \operatorname{opt}(\mathrm{TST}(G, c, R))-\left(\frac{\rho}{2}\right) c\left(F_{R}\right) \leqslant \beta \operatorname{opt}(T S T(G, c, R))
$$

então

$$
c(T) \leqslant \beta \operatorname{opt}(\mathrm{TST}(G, \mathrm{c}, \mathrm{R})) .
$$

Prova. Sejam $\delta, R^{\prime}$ e $S$ um desmembramento de custo mínimo, um conjunto de centros de estrelas de $S$ e uma árvore de Steiner construídos pelo ALGORITMO ÁRVORE 2 , respectivamente. Então,

$$
\begin{aligned}
c(T) & =c(S)+c(S) \\
& \leqslant \rho \operatorname{opt}\left(\operatorname{ST}\left(G-R, c, R^{\prime}\right)\right)+c(S) \\
& \leqslant \rho\left(\operatorname{opt}(\operatorname{TST}(G, c, R))+\frac{c(S)}{2}-\frac{c\left(F_{R}\right)}{2}\right)+c(S) \\
& =\rho \operatorname{opt}(\operatorname{TST}(G, c, R))+\left(\frac{\rho}{2}+1\right) c(S)-\left(\frac{\rho}{2}\right) c\left(F_{R}\right) \\
& \leqslant \rho \operatorname{opt}(\operatorname{TST}(G, c, R))+\left(\frac{\rho}{2}+1\right) \operatorname{opt}(T S T(G, c, R))-\left(\frac{\rho}{2}\right) c\left(F_{R}\right) \\
& =\left(\frac{3}{2} \rho+1\right) \operatorname{opt}(T S T(G, c, R))-\left(\frac{\rho}{2}\right) c\left(F_{R}\right) \\
& \leqslant \beta \operatorname{opt}(\operatorname{TST}(G, c, R)),
\end{aligned}
$$

onde a igualdade (2.10) vale devido ao lema 2.9, a desigualdade (2.11) segue do lema 2.10 e a desigualdade (2.12) vale porque $S$ é um desmembramento de custo mínimo.

Assim, o ALGORITMO ÁRVORE 2 constrói uma árvore de Steiner de terminais folhas que, sob certas condições como veremos na seção 2.2.3, é uma boa aproximação para uma árvore de Steiner de terminais folhas ótima. 


\subsubsection{Algoritmo Terminal}

Apresentamos nesta seção um algoritmo de aproximação de razão de aproximação $\alpha$ para o problema TST e a prova do teorema 2.2. O ALGORITMO TERMINAL executa O ALGORITMO ÁRVORE 1 e o ALGORITMO ÁRVORE 2 sobre a instância $G, c$ e R, recebe as árvores $T_{1}$ e $T_{2}$ resultantes das respectivas execuções e devolve a árvore de menor custo entre estas.

ALGORITMO TERMINAL: recebe um grafo completo $G$, uma função custo $c: E_{G} \rightarrow \mathbb{Q} \geqslant$ satisfazendo a desigualdade triangular e um conjunto $R \subseteq V_{G}$ de terminais, e devolve uma árvore de Steiner de terminais folhas $T$ tal que $c(T) \leqslant \alpha \operatorname{opt}(T S T(G, c, R))$.

1: Seja $T_{1}$ a árvore de Steiner de terminais folhas devolvida pelo ALGORITMO ÁRVORE 1 para a instância G, c e R do problema TST.

2: Seja $T_{2}$ a árvore de Steiner de terminais folhas devolvida pelo ALGORITMO ÁRVORE 2 para a instância $G, c$ e R do problema TST.

3: Seja $T$ a árvore de menor custo entre $T_{1}$ e $T_{2}$.

4: Devolva T e pare.

Agora podemos mostrar que o teorema 2.2 é válido. Nós comparamos o valor $c\left(F_{R}\right)$ da soma dos custos das arestas de menor custo que incidem em cada terminal de $R$ com o custo da árvore de Steiner de terminais folhas ótima para a instância $G, c$ e R. Se $c\left(F_{R}\right)$ é pequeno, de certo modo, comparado com opt $(T S T(G, c, R))$ então a árvore $T_{1}$ construída no ALGORITMO TERMINAl é uma boa aproximação. Caso contrário, a árvore $T_{2}$ é uma boa aproximação.

Prova do TEOREMA 2.2. Sejam $T_{1}$ e $T_{2}$ as árvores devolvidas pelo Algoritmo ÁrVOrE 1 e pelo ALGORITMO ÁRVORE2, respectivamente, para a instância G, c e R.

Como $c\left(F_{R}\right) \leqslant \operatorname{opt}(T S T(G, c, R))$, a hipótese do teorema 2.5 sempre é válida se tomamos $\beta:=\alpha_{2}$. Ou seja,

$$
\begin{aligned}
\rho \operatorname{opt}(\operatorname{TST}(G, c, R))+3(\rho-1) c\left(F_{R}\right) & \leqslant \rho \operatorname{opt}(\operatorname{TST}(G, c, R))+3(\rho-1) \operatorname{opt}(\operatorname{TST}(G, c, R)) \\
& =(4 \rho-3) \operatorname{opt}(\operatorname{TST}(G, c, R)) .
\end{aligned}
$$

Dessa forma, se tomamos $\beta=\alpha_{2}=4 \rho-3$, temos pelo teorema 2.5 que

$$
c\left(T_{1}\right) \leqslant \alpha_{2} \operatorname{opt}(\operatorname{TST}(G, c, R)) .
$$

Neste ponto, dividimos a análise em dois casos, dependendo se a desigualdade

$$
c\left(F_{R}\right) \leqslant\left(\frac{\rho+2}{7 \rho-6}\right) \operatorname{opt}(T S T(G, c, R))
$$


vale ou não. Se valer, então temos que

$$
\begin{aligned}
\operatorname{\rho opt}(\operatorname{TST}(G, c, R))+3(\rho-1) c\left(F_{R}\right) \leqslant & \rho \operatorname{opt}(\operatorname{TST}(G, c, R))+ \\
& \left(\frac{3(\rho-1)(\rho+2)}{7 \rho-6}\right) \operatorname{opt}(\operatorname{TST}(G, c, R)) \\
= & \left(\frac{10 \rho^{2}-3 \rho-6}{7 \rho-6}\right) \operatorname{opt}(\operatorname{TST}(G, c, R)) \\
= & \left(1+\frac{10 \rho(\rho-1)}{7 \rho-6}\right) \operatorname{opt}(\operatorname{TST}(G, c, R)) .
\end{aligned}
$$

Logo, se fizermos

$$
\beta:=\alpha_{1}=\left(1+\frac{10 \rho(\rho-1)}{7 \rho-6}\right)
$$

as hipóteses do teorema 2.5 são satisfeitas e isso implica que

$$
c\left(T_{1}\right) \leqslant \alpha_{1} \operatorname{opt}(\operatorname{TST}(G, c, R)) .
$$

Por outro lado, se

$$
c\left(F_{R}\right) \geqslant\left(\frac{\rho+2}{7 \rho-6}\right) \operatorname{opt}(\mathrm{TST}(G, c, R))
$$

então temos que

$$
\begin{aligned}
\left(\frac{3}{2} \rho+1\right) \operatorname{opt}(\operatorname{TST}(G, c, R))-\left(\frac{\rho}{2}\right) c\left(F_{R}\right) \leqslant & \left(\frac{3}{2} \rho+1\right) \operatorname{opt}(\operatorname{TST}(G, c, R))- \\
& \left(\frac{\rho}{2}\right)\left(\frac{\rho+2}{7 \rho-6}\right) \operatorname{opt}(\operatorname{TST}(G, c, R)) \\
= & \left(\frac{3}{2} \rho-\left(\frac{\rho}{2}\right)\left(\frac{\rho+2}{7 \rho-6}\right)+1\right) \operatorname{opt}(\operatorname{TST}(G, c, R)) \\
& \left(1+\frac{10 \rho(\rho-1)}{7 \rho-6}\right) \operatorname{opt}(\operatorname{TST}(G, c, R)) .
\end{aligned}
$$

Dessa forma, se fizermos

$$
\beta:=\alpha_{1}=\left(1+\frac{10 \rho(\rho-1)}{7 \rho-6}\right)
$$

as hipóteses do teorema 2.11 são satisfeitas e temos então que

$$
c\left(T_{2}\right) \leqslant \alpha_{1} \operatorname{opt}(\operatorname{TST}(G, c, R)) .
$$

Portanto, como o ALGORITMO TERMINAL devolve a árvore $T$ de menor custo entre $T_{1}$ e $T_{2}$, concluímos que

$$
c(T) \leqslant \alpha \operatorname{opt}(T S T(G, c, R))
$$

onde $\alpha=\min \left\{\alpha_{1}, \alpha_{2}\right\}$. 


\subsection{Uma 2,52-aproximação para o problema TST}

Sejam G, c e R uma instância do problema TST. Como na seção anterior, definimos para cada vértice terminal $r$ em $R$ a aresta $e_{r}$ como uma aresta de menor custo conectando $r$ a $V_{G} \backslash R$ e $F_{R}$ um conjunto $\left\{e_{r}: r \in R\right\}$. Descrevemos nesta seção um algoritmo de aproximação que recebe uma instância $G$, c e R do problema TST e constrói duas árvores de Steiner de terminais folhas $T_{1}$ e $T_{2}$ tais que se o valor de $c\left(F_{R}\right)$ é 'pequeno' comparado ao valor $\operatorname{de~} \operatorname{opt}(\operatorname{TST}(G, c, R)$ ) então temos que

$$
c\left(T_{1}\right) \leqslant \alpha \operatorname{opt}(\operatorname{TST}(G, c, R))
$$

caso contrário,

$$
c\left(T_{2}\right) \leqslant \alpha \operatorname{opt}(\operatorname{TST}(G, c, R)) \text {. }
$$

O algoritmo devolve a árvore de Steiner de terminais folhas de menor custo entre as árvores construídas.

Da mesma forma que antes, como o problema TST é trivial para $|R| \leqslant 2$, podemos considerar que $|R| \geqslant 3$. Para $|R| \geqslant 3$, como uma árvore de Steiner de terminais folhas não contém arestas conectando terminais, podemos considerar que para cada par de vértices $q, r$ em $R$, o custo da aresta qr é $\min _{v \in V_{G} \backslash R}(c(q v)+c(v r))$. Em particular, isso implica que podemos considerar que toda árvore de Steiner para uma instância $G$, c e R não contém arestas contectando vértices terminais.

O resultado principal que queremos mostrar nesta seção é enunciado a seguir.

TEOREMA 2.12 Existe um algoritmo de aproximação de tempo polinomial para o problema TST com razão de aproximação $\alpha$ tal que

$$
\alpha=2 \rho-\left(\frac{\rho}{3 \rho-2}\right)
$$

supondo que um algoritmo de aproximação de tempo polinomial obtém uma razão de aproximação p para o problema ST.

Como o melhor valor conhecido para $\rho$ é ligeiramente menor que 1,55 o teorema 2.12 implica o seguinte corolário.

COROLÁRIO 2.13 Existe uma 2,52-aproximação para o problema TST.

Como na seção 2.2.1, construímos uma árvore de Steiner de terminais folhas $T_{1}$ na tentativa que essa árvore seja uma boa aproximação para o problema TST. Do mesmo modo, modificamos os custos das arestas que incidem em vértices terminais da instância de entrada, uma árvore de Steiner é construída para essa instância modificada e, finalmente, substituições de arestas são realizadas sobre essa árvore de Steiner de tal forma que o algoritmo obtém uma árvore de Steiner de terminais folhas. Pela proposição 2.4 na seção 2.2, sabemos então que essa árvore resultante é, sob certas hipóteses, uma boa aproximação para uma árvore de Steiner de terminais folhas de custo ótimo. 
Essa transformação que enfatizamos pode não ser suficiente para garantir uma boa aproximação para o problema TST. Então, diferentemente dessa transformação descrita na seção 2.2.2, um outro tipo de transformação é realizado sobre uma árvore de Steiner para a instância $G, c$ e R. Para cada terminal em $R$ que tem mais de dois vizinhos na árvore de Steiner construída, tomamos um caminho induzido por esses vizinhos na árvore de Steiner e substituímos todas as arestas da árvore que incidem no terminal, menos aquela de menor custo, pelas arestas desse caminho induzido. Esse processo se repete para todo terminal na árvore de Steiner e, no final, temos uma árvore de Steiner de terminais folhas para a instância de entrada satisfazendo a seguinte propriedade.

LEMA 2.14 Seja G, c e R uma instância do problema TST e seja $\mathrm{F}_{\mathrm{R}}=\left\{\mathrm{e}_{\mathrm{r}}: \mathrm{r} \in \mathrm{R}\right\}$ um conjunto das arestas de menor custo que incidem nos terminais. Dada uma árvore de Steiner $\mathrm{S}$ podemos encontrar em tempo polinomial uma árvore de Steiner de terminais folhas T tal que

$$
c(T) \leqslant 2 c(S)-c\left(F_{R}\right)
$$

PROVA. Se cada vértice em $R$ é uma folha na árvore $S$, então fazemos $T:=S$ e o lema está provado. Agora, suponha que existe um vértice $r$ em $R$ tal que seu conjunto $W$ de vizinhos em $S$ tem mais que um vértice. Seja $u$ um vértice em $V_{G} \backslash\{r\}$ tal que $e_{r}=r u$. Seja $X=\{r w: w \in W\}$ um conjunto de arestas incidentes no vértice $r$ e sejam $r x$ e ry duas arestas de maior custo em $X$. Seja $Y$ um conjunto de arestas de um $x y$-caminho no subgrafo induzido pelos vértices de $W$. Veja a figura 2.7 para uma ilustração.

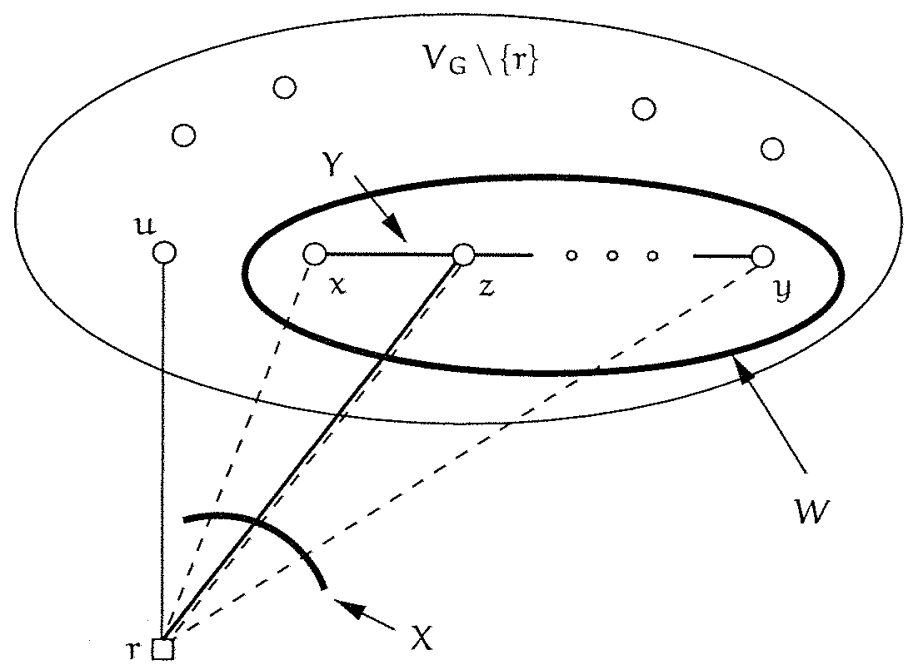

Figura 2.7: Ilustração para o lema 2.14. Arestas pontilhadas são removidas e arestas sólidas são incluídas. O vértice u pode pertencer a $W$.

Pela desiguladade triangular temos que

$$
c(Y) \leqslant 2 c(X)-c(r x)-c(r y) .
$$


Finalmente, seja $S^{\prime}$ a árvore de Steiner induzida pelas arestas em $\left(E_{S} \backslash X\right) \cup Y \cup\{r z\}$, onde $r z$ é uma aresta de menor custo em $X$. Temos então que

$$
\begin{aligned}
c\left(S^{\prime}\right) & =c\left(E_{S}\right)-c(X)+c(Y)+c(r z) \\
& \leqslant c\left(E_{S}\right)-c(X)+2 c(X)-c(r x)-c(r y)+c(r z) \\
& \leqslant c\left(E_{S}\right)+c(X)-c(r x) \\
& \leqslant c\left(E_{S}\right)+c(X)-c(r u) \\
& =c(S)+c(X)-c(r u)
\end{aligned}
$$

onde a desigualdade (2.14) segue da desigualdade (2.13). Observe que $S^{\prime}$ é uma árvore de Steiner onde $r$ é folha e os terminais folhas na árvore $S$ permanecem folhas em $S^{\prime}$. Repetindo esse processo, obtemos uma árvore de Steiner de terminais folhas satisfazendo o lema.

Apresentamos a seguir um algoritmo de aproximação com razão de aproximação $\alpha$ para o problema TST e a prova do teorema 2.12. O ALGORITMO TERMINAL constrói duas árvores de Steiner de terminais folhas para a instância $G, c$ e R: uma árvore $T_{1}$ através da modificação dos custos das arestas de $G$ e da substituição de certas arestas de uma árvore de Steiner nessa instância modificada e uma árvore $T_{2}$ através da substituição de outras arestas de uma árvore de Steiner na instância de entrada, conforme o lema 2.14.

ALGORITMO TERMINAL : recebe um grafo completo $G$, uma função custo $c: E_{G} \rightarrow \mathbb{Q} \geqslant$ satisfazendo a desigualdade triangular e um conjunto $R \subseteq V_{G}$ de terminais, e devolve uma árvore de Steiner de terminais folhas $T$ tal que $c(T) \leqslant \alpha \operatorname{opt}(T S T(G, c, R)$ ).

1: Seja c': $E_{G} \rightarrow \mathbb{Q} \geqslant$ definida como

$$
c^{\prime}(e):= \begin{cases}c(e)+3 c\left(e_{q}\right)+3 c\left(e_{r}\right), & \text { se } e \cap R=\{q, r\} \\ c(e)+3 c\left(e_{r}\right), & \text { se } e \cap R=\{r\} \\ c(e), & \text { se } e \cap R=\emptyset\end{cases}
$$

2: Seja $S_{1}$ uma árvore de Steiner devolvida por uma $\rho$-aproximação para o problema ST com instância $G, c^{\prime}$ e R.

3: Construa uma árvore de Steiner de terminais folhas $T_{1}$ a partir de $S_{1}$ usando a proposição 2.4.

4: Seja $S_{2}$ a árvore de Steiner devolvida por uma $\rho$-aproximação para o problema ST com instância $G, c$ e R.

5: Construa uma árvore de Steiner de terminais folhas $T_{2}$ a partir de $S_{2}$ usando o lema 2.14.

6: Seja $T$ a árvore de menor custo entre $T_{1}$ e $T_{2}$.

7: Devolva T e pare.

Prova do TEOREMA 2.12. Sejam $c^{\prime}, S_{1}, S_{2}, T_{1}$ e $T_{2}$ a função custo, as árvores de Steiner e as árvores de Steiner de terminais folhas construídas pelo ALGORITMO TERMINAL` para uma instância G, c e R. Suponha primeiro que

$$
c\left(F_{R}\right) \leqslant\left(\frac{\rho}{3 \rho-2}\right) \operatorname{opt}(T S T(G, c, R)) .
$$


Como $c^{\prime}$ satisfaz a hipótese da proposição 2.4 , então sabemos que $c^{\prime}\left(T_{1}\right) \leqslant c^{\prime}\left(S_{1}\right)$. Além disso, para cada $r$ em $\mathrm{R}$ a aresta incidente a $r$ em $T_{1}$ é $e_{r}$. Dessa forma,

$$
\begin{aligned}
c\left(T_{1}\right) & =c^{\prime}\left(T_{1}\right)-3 c\left(F_{R}\right) \\
& \leqslant c^{\prime}\left(S_{1}\right)-3 c\left(F_{R}\right) \\
& \leqslant \rho \operatorname{opt}\left(\operatorname{ST}\left(G, c^{\prime}, R\right)\right)-3 c\left(F_{R}\right) \\
& \leqslant \rho\left(\operatorname{opt}(T S T(G, c, R))+3 c\left(F_{R}\right)\right)-3 c\left(F_{R}\right) \\
& =\rho \operatorname{opt}(\operatorname{TST}(G, c, R))+3(\rho-1) c\left(F_{R}\right) \\
& \leqslant \rho \operatorname{opt}(T S T(G, c, R))+3(\rho-1)\left(\frac{\rho}{3 \rho-2}\right) \operatorname{opt}(T S T(G, c, R)) \\
& \leqslant \alpha \operatorname{opt}(T S T(G, c, R)),
\end{aligned}
$$

onde a desigualdade (2.15) vale já que se $T^{*}$ é uma árvore de Steiner de terminais folhas tal que $c\left(T^{*}\right)=\operatorname{opt}(T S T(G, c, R))$, então

$$
\operatorname{opt}\left(S T\left(G, c^{\prime}, R\right)\right) \leqslant c^{\prime}\left(T^{*}\right)=c\left(T^{*}\right)+3 c\left(F_{R}\right)=\operatorname{opt}(T S T(G, c, R))+3 c\left(F_{R}\right) .
$$

Agora, suponha que

$$
c\left(F_{R}\right) \geqslant\left(\frac{\rho}{3 \rho-2}\right) \operatorname{opt}(\operatorname{TST}(G, c, R))
$$

Seja $S_{2}$ a árvore de Steiner construída pelo algoritmo. Então,

$$
\begin{aligned}
c\left(T_{2}\right) & \leqslant 2 c\left(S_{2}\right)-c\left(F_{R}\right) \\
& \leqslant 2 \rho \operatorname{opt}(S T(G, c, R))-c\left(F_{R}\right) \\
& \leqslant 2 \rho \operatorname{opt}(\operatorname{TST}(G, c, R))-c\left(F_{R}\right) \\
& \left.\leqslant 2 \rho \operatorname{opt}(\operatorname{TST}(G, c, R))-\left(\frac{\rho}{3 \rho-2}\right) \operatorname{opt}(\operatorname{TST}(G, c, R))\right) \\
& \leqslant \alpha \operatorname{opt}(\operatorname{TST}(G, c, R)),
\end{aligned}
$$

onde a desigualdade (2.16) vale devido ao lema 2.14.

Finalmente, como o ALGORITMO TERMINAL ${ }^{\star}$ devolve a árvore $T$ de menor custo entre $T_{1} \mathrm{e}$ $\mathrm{T}_{2}$, concluímos que

$$
c(T) \leqslant \alpha \operatorname{opt}(T S T(G, c, R))=\left(2 \rho-\left(\frac{\rho}{3 \rho-2}\right)\right) \operatorname{opt}(T S T(G, c, R)),
$$

supondo que um algoritmo de aproximação de tempo polinomial obtém uma razão de aproximação $\rho$ para o problema ST.

$\mathrm{Na}$ figura 2.8 mostramos um gráfico com as razões de aproximação obtidas pelo ALGORITMO TERMINAL e pelo ALGORITMO TERMINAL em função de $\rho$, a razão de aproximação obtida para o problema ST. 


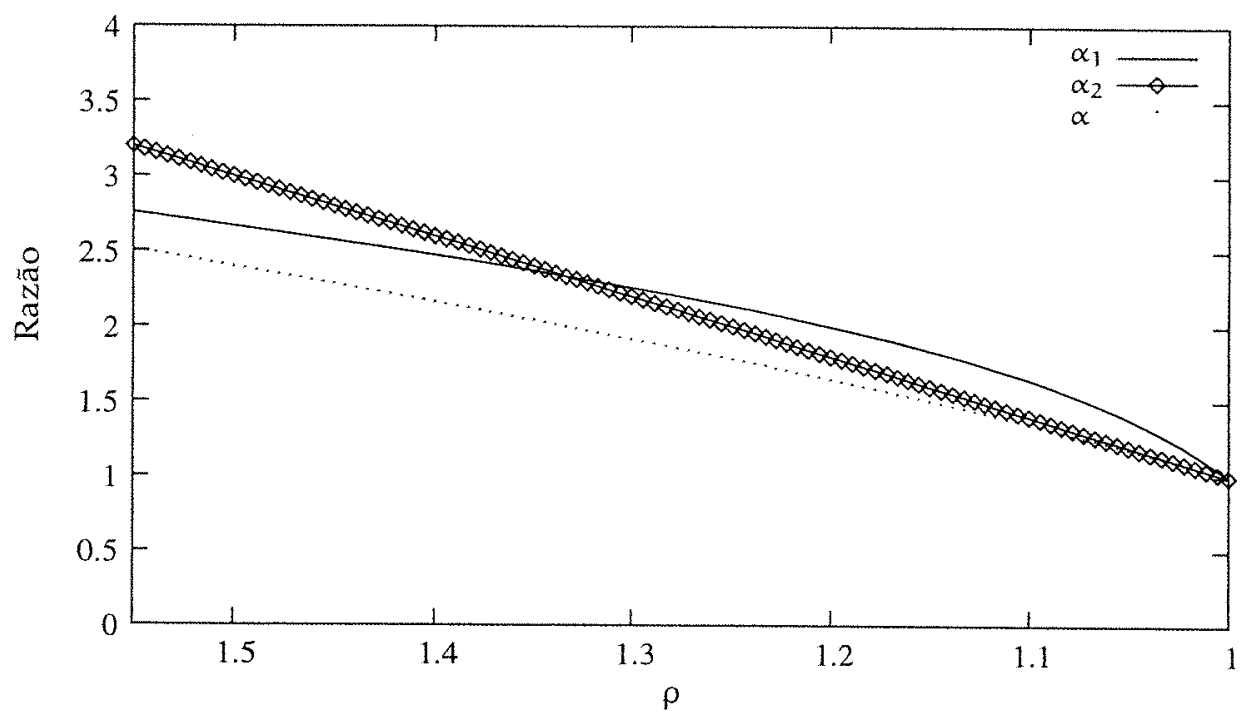

Figura 2.8: Razões de aproximação obtidas para o problema TST em função de $\rho$, onde $\alpha_{1}$ e $\alpha_{2}$ são obtidas pelo algoritmo ALGORITMO TERMINAL e $\alpha$ é obtida pelo algoritmo ALGORITMO TERMINAL ${ }^{\star}$.

\subsection{APX-completude para o problema TST}

Do resultado de Lu et al. (2003) mostrando que o problema TST $_{1,2}$ é MAXSNP-difícil decorre que o problema TST é MAXSNP-difícil. Mencionamos novamente esse resultado de forma apropriada no capítulo 3, quando tratamos do problema TST $_{1,2}$. Apresentamos nesta seção uma AP-redução do problema ST para o problema TST e assim, como o problema ST é APX-completo (Gröpl et al., 2001), mostramos que o problema TST é APX-completo.

TEOREMA 2.15 O problema TST é APX-completo.

ProvA. Nas seções 2.1, 2.2 e 2.3 mostramos que existe uma $\alpha$-aproximação para o problema TST, onde $\alpha$ é uma constante. Dessa forma, o problema TST está em APX.

Para mostrar que o problema TST é APX-difícil, faremos uma AP-redução do problema ST para o problema TST, isto é, ST $\leqslant$ AP TST. Devemos então determinar uma tripla $(f, g, \beta)$ satisfazendo (AP1), (AP2), (AP3) e (AP4) como definido na seção 1.2.

A função f recebe um racional positivo $\delta$ e uma instância $G$, c e R do problema ST e produz uma instância $\left(G^{\prime}, c^{\prime}, R^{\prime}\right):=f(\delta,(G, c, R))$ do problema TST da seguinte forma. Dado $R:=$ $\left\{r_{1}, r_{2}, \ldots, r_{|R|}\right\}, f$ constrói um conjunto $R^{\prime}:=\left\{r_{1}^{\prime}, r_{2}^{\prime}, \ldots, r_{|R|}^{\prime}\right\}$. Então, o grafo $G^{\prime}$ é definido como o grafo completo com $V_{G^{\prime}}:=V_{G} \cup R^{\prime}$. A função custo $c^{\prime}: E_{G^{\prime}} \rightarrow \mathbb{Q} \geqslant$ é definida como

$$
c^{\prime}(u v):= \begin{cases}c(u v), & \text { se } u, v \in V_{G^{\prime}} \backslash R^{\prime}=V_{G} \\ \mathcal{E}, & \text { se existe } i \text { tal que } u=r_{i} \text { e } v=r_{i}^{\prime} \\ c\left(r_{i} r_{j}\right)+2 \varepsilon, & \text { se existem } i, j, i \neq j, \text { tais que } r_{i}, r_{j} \in R \text { e } u, v \in R^{\prime} \\ c\left(u r_{i}\right)+\varepsilon, & \text { se existe } i \text { tal que } r_{i} \in R, u \in V_{G^{\prime}} \backslash\left(R \cup R^{\prime}\right) \text { e } v=r_{i}^{\prime} \in R^{\prime},\end{cases}
$$


onde $\varepsilon$ é a metade do menor valor de $c(u v)$ para $u v \in E_{G}$. Ou seja, $\varepsilon:=\min _{u v \in E_{G}} c(u v) / 2$. A figura 2.9 mostra um exemplo da construção da função $f$.

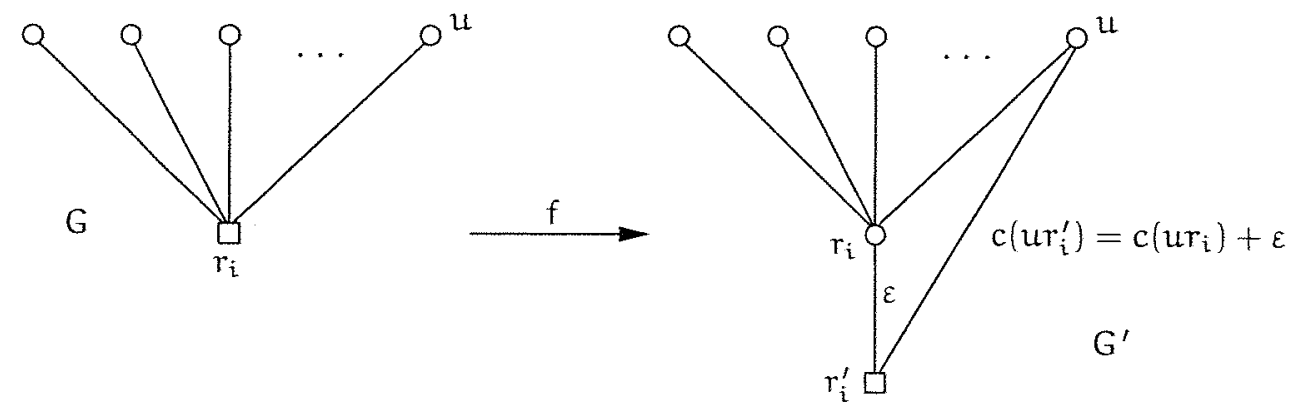

Figura 2.9: Transformação de um terminal em $G$ pela função $f$.

A função $g$ recebe um racional positivo $\delta$, uma instância $G, c$ e $R$ do problema ST e uma solução $T^{\prime}$ do problema TST para a instância $G^{\prime}, c^{\prime}$ e $R^{\prime}$ e transforma $T^{\prime}$ em $T$, uma solução para o problema ST. Para cada $r^{\prime}$ em $T^{\prime}$, temos que ou $r^{\prime}$ é adjacente a um vértice de Steiner $r$ em $T^{\prime}$, com $r \in R$, ou $r^{\prime}$ é adjacente a um vértice de Steiner s em $T^{\prime}$, com $s \notin R$. Remova de $T^{\prime}$ todas as arestas $r r^{\prime}$ e os terminais $r^{\prime}$ em $R^{\prime}$. Remova também as arestas $s r^{\prime}$, os terminais $r^{\prime}$ em $R^{\prime}$ e adicione a aresta sr de $E_{G}$, para algum $r$ em $R$. Chame de $T$ essa estrutura, com

$$
\begin{aligned}
V_{T}:= & V_{T^{\prime}} \backslash R^{\prime} \\
E_{T}:= & \left(E_{T^{\prime}} \backslash\left(\left\{r r^{\prime} \in E_{T^{\prime}}: r \in R \text { er } r^{\prime} \in R^{\prime}\right\} \cup\left\{s r^{\prime} \in E_{T^{\prime}}: s \in V_{G^{\prime}} \backslash\left(R \cup R^{\prime}\right) \text { e } r^{\prime} \in R^{\prime}\right\}\right)\right) \cup \\
& \left\{s r \in E_{G}: s \in V_{G^{\prime}} \backslash\left(R \cup R^{\prime}\right) \text { e } r \in R\right\} .
\end{aligned}
$$

A figura 2.10 mostra um exemplo do funcionamento da função $\mathrm{g}$.

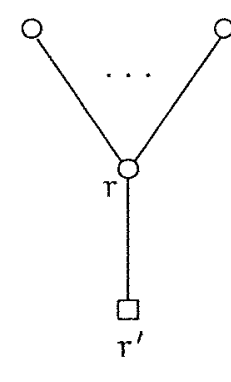

$T^{\prime}$

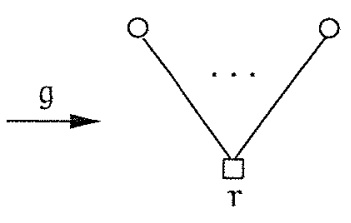

$\mathrm{T}$

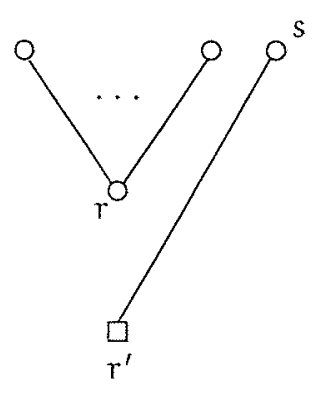

$T^{\prime}$

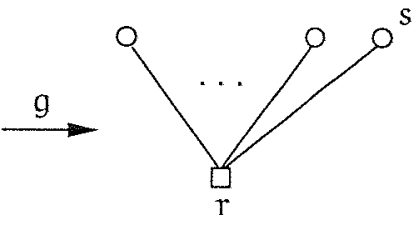

T

Figura 2.10: Transformações sobre a árvore $T^{\prime}$ realizadas pela função $g$.

Note agora que as funções $f$ e $g$ que acabamos de definir são transformações polinomiais sobre as suas respectivas entradas. A figura 2.11 mostra um esquema de funcionamento dessas transformações $\mathrm{f}$ e $\mathrm{g}$. 
ST

TST
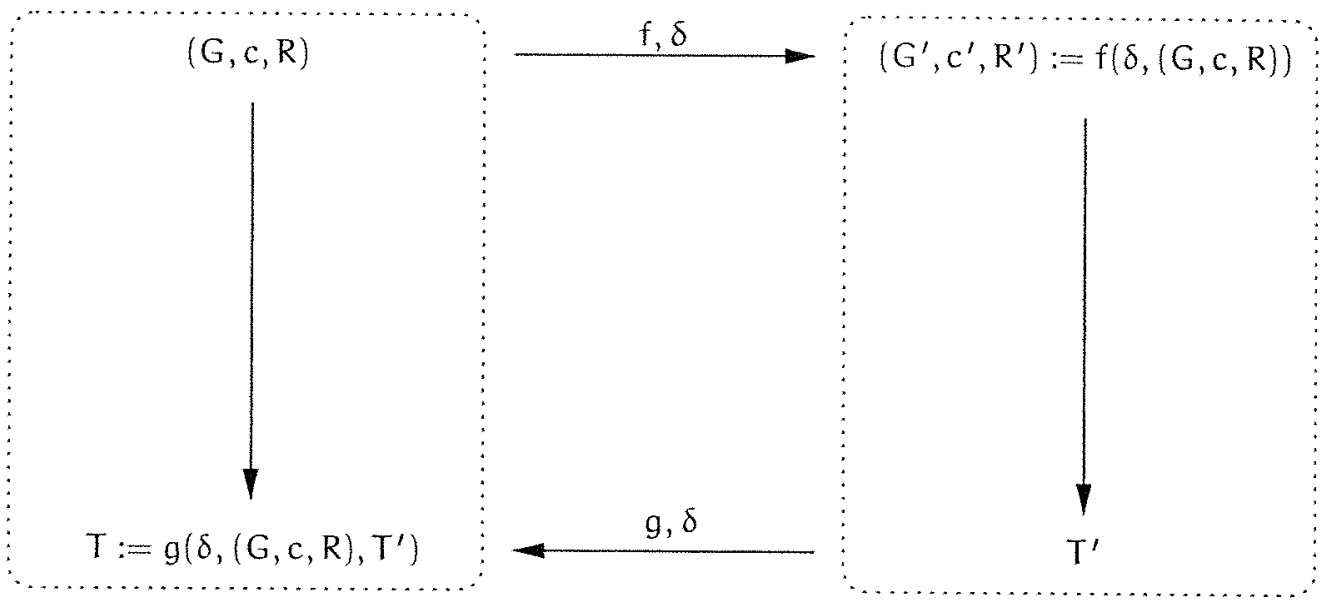

Figura 2.11: Um esquema da AP-redução do problema ST para o problema TST, evidenciando as transformações $f \mathrm{e} g$.

Vamos supor que para alguma instância G, c e R do problema ST, algum racional positivo $\delta$ e alguma solução $T^{\prime}$ do problema TST para a instância $\left(G^{\prime}, c^{\prime}, R^{\prime}\right):=f(\delta,(G, c, R))$ vale que

$$
(1-\delta) \operatorname{opt}\left(T S T\left(G^{\prime}, c^{\prime}, R^{\prime}\right)\right) \leqslant c^{\prime}\left(T^{\prime}\right) \leqslant(1+\delta) \operatorname{opt}\left(T S T\left(G^{\prime}, c^{\prime}, R^{\prime}\right)\right)
$$

Como os problemas envolvidos são de minimização, a desigualdade direita é a que interessa. Queremos provar que a árvore $T:=g\left(\delta,(G, c, R), T^{\prime}\right)$, construída pela função $g$, é tal que

$$
c(T) \leqslant(1+\beta \delta) \operatorname{opt}(S T(G, c, R))
$$

para algum $\beta$ fixo. Vamos escolher para esta redução o valor de $\beta:=2$. Observe que da forma como as funções $f$ e $g$ foram definidas, temos que

$$
c^{\prime}\left(T^{\prime}\right)=c(T)+\varepsilon|R|
$$

Em particular,

$$
\operatorname{opt}\left(T S T\left(G^{\prime}, c^{\prime}, R^{\prime}\right)\right)=\operatorname{opt}(S T(G, c, R))+\varepsilon|R|
$$

Então,

$$
\begin{aligned}
c^{\prime}\left(T^{\prime}\right) & \leqslant(1+\delta) \operatorname{opt}\left(\operatorname{TST}\left(G^{\prime}, c^{\prime}, R^{\prime}\right)\right) \\
c(T)+\varepsilon|R| & \leqslant(1+\delta) \operatorname{opt}(S T(G, c, R))+\varepsilon|R|) \\
c(T) & \leqslant(1+\delta) \operatorname{opt}(S T(G, c, R))+((1+\delta) \varepsilon-\varepsilon)|R| \\
& =(1+\delta) \operatorname{opt}(\operatorname{ST}(G, c, R))+\delta \varepsilon|R|
\end{aligned}
$$


Agora, se denotamos por $c_{e}$ o custo de uma aresta de menor custo em $E_{G}$ e, lembrando que $\varepsilon=c_{e} / 2$, então

$$
\begin{aligned}
\operatorname{opt}(S T(G, c, R)) & \geqslant(|R|-1) c_{e} \\
& =(|R|-1) 2 \varepsilon \\
& \geqslant \varepsilon|R| .
\end{aligned}
$$

Assim,

$$
\begin{aligned}
c(T) & \leqslant(1+\delta) \operatorname{opt}(S T(G, c, R))+\delta \varepsilon|R| \\
& \leqslant(1+\delta) \operatorname{opt}(S T(G, c, R))+\delta \operatorname{opt}(S T(G, c, R)) \\
& =(1+2 \delta) \operatorname{opt}(S T(G, c, R)) \\
& =(1+\beta \delta) \operatorname{opt}(S T(G, c, R)) .
\end{aligned}
$$

Portanto, temos que ST \$AP TST, e como o problema ST é APX-completo (Gröpl et al., 2001), temos que o problema TST é APX-completo.

Em decorrência dos teoremas 1.1 e 1.4, temos como uma conseqüência do teorema 2.15 que não há um esquema de aproximação polinomial para o problema TST, a menos que $\mathrm{P}=\mathrm{NP}$. 


\section{Capítulo 3}

\section{Problema $\mathrm{TST}_{1,2}$}

O problema $\mathrm{TST}_{1,2}$ é uma restrição do problema TST, apresentado no capítulo 2 , onde os custos das arestas do grafo de uma instância são 1 ou 2. Observe que a função custo mencionada satisfaz a desigualdade triangular. Formalmente,

Problema $\mathrm{TST}_{1,2}(\mathrm{G}, \mathrm{c}, \mathrm{R})$ : dado um grafo completo $\mathrm{G}$, uma função custo $c: E_{G} \rightarrow\{1,2\}$ e um conjunto $R \subseteq V_{G}$ de terminais, encontrar uma árvore de Steiner de terminais folhas de custo mínimo em $\mathrm{G}$.

A motivação inicial para a investigação do problema $\mathrm{TST}_{1,2}$ é essencialmente teórica, embora podemos pensar em aplicações como, por exemplo, quando somente dois tipos de conexão podem ser realizadas entre os vértices de uma instância, uma barata e outra cara, e, do mesmo modo, queremos encontrar uma árvore de Steiner de terminais folhas de custo mínimo para essa instância. Como antes, o problema $\mathrm{TST}_{1,2}$ está relacionado ao problema $\mathrm{ST}_{1,2}$, que por sua vez é uma restrição do problema ST. Chamamos de problema $\mathrm{ST}_{1,2}$ o problema ST com custos das arestas restritos a 1 ou 2 . Bern \& Plassmann (1989) propuseram uma 4/3-aproximação para o problema $\mathrm{ST}_{1,2}$, através do uso da heurística do custo médio (Rayward-Smith, 1983), e mostraram que o problema é MAXSNP-difícil, fazendo uma L-redução do problema da cobertura de vértices. Robins \& Zelikovsky (2000) propuseram um outro algoritmo de aproximação e melhoraram essa razão para 1,28. Lu et al. (2003) adaptaram o algoritmo de aproximação e a L-redução de Bern \& Plassmann (1989) para o problema TST 1,2 . Dessa forma, obtiveram uma 8/5-aproximação e mostraram que o problema TST 1,2 é MAXSNP-difícil. Apresentamos neste capítulo uma 17/12-aproximação para o problema $\mathrm{TST}_{1,2}$, melhorando ${ }^{1}$ assim a razão de aproximação estabelecida pelo algoritmo de Lu et al. (2003). Mostramos ainda que o problema $\mathrm{TST}_{1,2}$ é APX-completo através de uma AP-redução do problema $\mathrm{ST}_{1,2}$. Esses resultados foram obtidos conjuntamente com o professor José Coelho de Pina Júnior.

Um algoritmo ingênuo é apresentado na seção 3.1 com razão de aproximação 2. Na seção 3.2, apresentamos o algoritmo de Lu et al. (2003). Na seção 3.3 propomos uma 17/12aproximação para o problema $\mathrm{TST}_{1,2}$ e na seção 3.4 mostramos o resultado de completude.

\footnotetext{
${ }^{1} 17 / 12=1,4166 \ldots<1,6=8 / 5$.
} 


\subsection{Uma 2-aproximação para o problema $\mathrm{TST}_{1,2}$}

Antes de apresentar aproximações mais elaboradas para o problema $\mathrm{TST}_{1,2}$, mostramos a seguir um algoritmo simples que obtém razão de aproximação 2 para toda instância $G, c$ e $R$ do problema $T_{S T} T_{1,2}$. O algoritmo toma uma instância $G, c$ e $R$ do problema, escolhe um vértice qualquer $s$ em $V_{G} \backslash R$, liga $s$ a cada terminal em $R$ e devolve esse subgrafo $T$ como resultado. Observe que T é uma árvore de Steiner de terminais folhas para G, c e R. Apresentamos a seguir o ALGORITMO INGÊNUO 1,2 .

ALGORITMO INGÊNUO 1,2 : recebe um grafo completo $G$, uma função custo $c: E_{G} \rightarrow\{1,2\}$ e um conjunto $\mathrm{R} \subseteq \mathrm{V}_{\mathrm{G}}$ de terminais, e devolve uma árvore de Steiner de terminais folhas.

1: Seja $s$ um vértice em $V_{G} \backslash R$.

2: Para cada $r$ em $R$, construa $T$ ligando $r$ a s.

3: Devolva Te pare.

A figura 3.1 mostra um exemplo de funcionamento do ALGORITMO INGÊNUO ${ }_{1,2}$ para uma instância G, c e R com dois vértices de Steiner conectados a vários terminais.

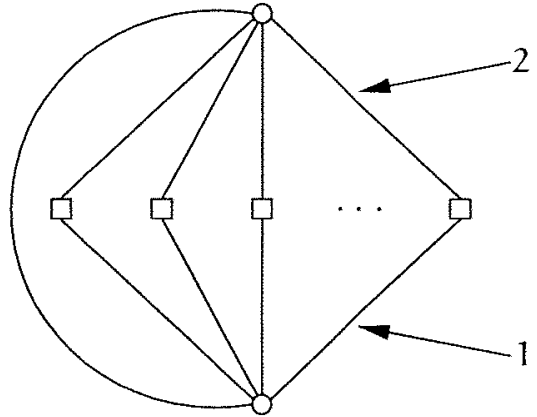

(a)

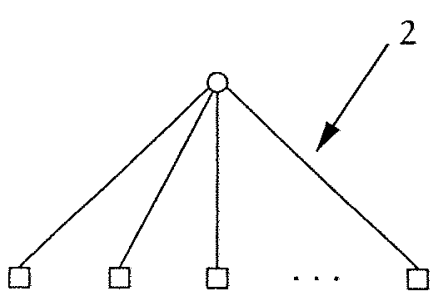

(b)

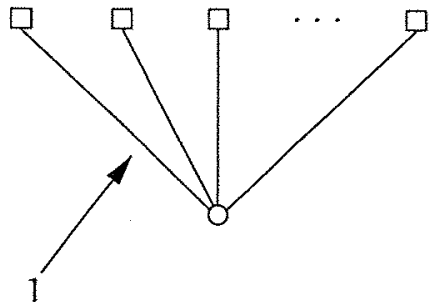

(c)

Figura 3.1: (a) uma instância $G_{,}$c e R do problema $\operatorname{TST}_{1,2}$; (b) uma árvore devolvida pelo ALGORITMO INGÊNUO 1,$2 ;$ (c) a árvore ótima para a instância.

ProposiçÃo 3.1 O ALGORITMO INGÊNUO ${ }_{1,2}$ é uma 2-aproximação para o problema TST $_{1,2}$.

Prova. Sejam G,c e R uma instância do problema $T_{S T} T_{1,2}$ e $T$ a árvore devolvida pelo ALGORITMO INGÊNUO 1,2 para essa instância. É fácil ver que $c(T) \leqslant 2|R|$ e ainda que $\operatorname{opt}\left(\mathrm{TST}_{1,2}(\mathrm{G}, \mathrm{c}, \mathrm{R})\right) \geqslant|R|$. Portanto,

$$
c(T) \leqslant 2|R| \leqslant 2 \operatorname{opt}\left(\operatorname{TST}_{1,2}(G, c, R)\right)
$$




\subsection{Uma 8/5-aproximação para o problema TST $_{1,2}$}

Como já mencionado, o algoritmo de Lu et al. (2003) usa a heurística do custo médio (Rayward-Smith, 1983) para obter uma árvore de Steiner de terminais folhas para uma instância do problema TST $_{1,2}$ com custo no máximo $8 / 5$ vezes maior que o custo de uma árvore de Steiner de terminais folhas ótima. Esse algoritmo é uma adaptação do algoritmo de Bern \& Plassmann (1989) para o problema $\mathrm{ST}_{1,2}$.

Sejam $G$, c e $R$ uma instância do problema $T_{S T} T_{1,2}$ e $u$ em $V_{G} \backslash R$ um vértice de Steiner de G. Uma estrela de terminais $\mathcal{S}_{\mathfrak{u}}$ é um subgrafo de $\mathrm{G}$ gerado pelo vértice $u$ e pelos terminais ligados a $u$. Chamamos de $R_{u} \subseteq R$ esses terminais. $O$ vértice $u$ é chamado centro da estrela $\mathcal{S}_{\mathcal{u}}$. Observe que a definição de estrela (de Steiner) apresentada no capítulo 2 é diferente da definição de estrela de terminais: no capítulo 2, a estrela é composta por um vértice terminal ligado a vértices de Steiner da instância do problema; aqui, a estrela de terminais é composta por um centro, que é um vértice de Steiner, ligado a vértices terminais. A figura 3.2 mostra um exemplo para essas definições.

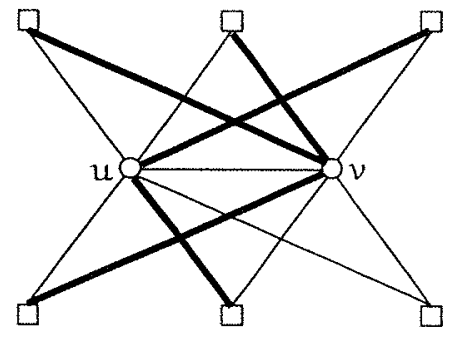

(a)

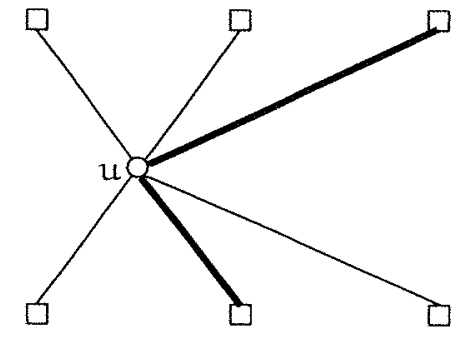

(b)

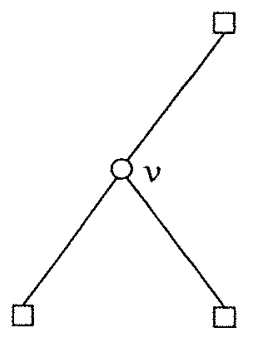

(c)

Figura 3.2: (a) uma instância G, c e R onde arestas finas têm custo 1, arestas grossas custo 2 e não são apresentadas as arestas entre os terminais; (b) uma estrela de terminais de centro $u$; (c) uma $\mathcal{X}_{3}$-estrela de centro $v$.

Para cada estrela de terminais $\mathcal{S}_{\mathrm{u}}$ em $\mathrm{G}$ tal que $\left|R_{u}\right| \geqslant 2$, definimos o seu custo médio $f\left(\mathcal{S}_{\mathrm{u}}\right)$ da seguinte forma:

$$
f\left(\mathcal{S}_{u}\right):=\frac{\sum_{r \in R_{u}} c(r u)}{\left|R_{u}\right|-1}
$$

Se $\mathcal{S}_{u}$ é uma estrela de terminais tal que $\mathrm{c}(\mathrm{ru})=1$ para todo terminal $\mathrm{r}$ em $R_{u}$, chamamos $\mathcal{S}_{\mathfrak{u}}$ de uma $\mathcal{X}_{\mathrm{k}}$-estrela, onde $\mathrm{k}:=\left|\mathrm{R}_{\mathfrak{u}}\right|$. A figura 3.2(c) mostra uma $\mathcal{X}_{3}$-estrela para a instância representada na figura 3.2(a).

O ALGORITMO CUSTOMÉdio é um algoritmo guloso que sempre seleciona uma $\mathcal{X}_{k}$-estrela de máximo $k, c o m k \geqslant 2$, para realizar uma redução na instância de entrada, exceto possivelmente na última iteração. Observe que uma $\mathcal{X}_{\mathrm{k}}$-estrela com $\mathrm{k}$ máximo em $\mathrm{G}$ é selecionada nas linhas de 2 a 7 do ALGORITMO CUSTOMÉdio e essa $\mathcal{X}_{k}$-estrela tem custo médio mínimo. Observe também que se $\mathcal{S}_{\mathfrak{u}}$ tem exatamente 2 terminais em $\mathrm{R}_{\mathfrak{u}}$ ligados a $u$ por arestas de custo 1 , então $f\left(\mathcal{S}_{u}\right)=2$. Nas linhas de 9 a 11 , o algoritmo faz uma redução: substitui a $\mathcal{X}_{k}$-estrela de $G$ por um vértice terminal $t$ ligado aos vértices restantes de $G$ e o custo de cada aresta que liga 
$\mathrm{t}$ a um vértice $v$ em $G-\mathcal{S}_{u}+\mathrm{t}$ é o custo da aresta que liga $u$ ao vértice $v$ em $G$. O ALGORITMO CusToMédio proposto por Lu et al. (2003) é apresentado a seguir.

ALGORITMO CUSTOMÉDIO: recebe um grafo completo $G$, uma função custo $c: E_{G} \rightarrow\{1,2\}$ e um conjunto $R \subseteq V_{G}$ de terminais, e devolve uma árvore de Steiner de terminais folhas.

1: $T \leftarrow \emptyset$.

2: se $V_{G} \backslash R$ contém pelo menos dois vértices de Steiner então

3: encontre uma estrela de terminais $\mathcal{S}_{\mathrm{u}}$ em $\mathrm{G}$ com menor custo médio.

4: $\quad$ se $f\left(\mathcal{S}_{u}\right)=2$ então

5: $\quad$ Remova os terminais de $\mathcal{S}_{u}$ que estão ligados a u por arestas de custo 2 .

6: senão

7: $\quad$ Seja $\mathcal{S}_{\mathfrak{u}}$ a estrela de terminais com centro $u$ ligado a todos os terminais de $\mathrm{R}$.

8: Adicione $\mathcal{S}_{\mathfrak{u}}$ a $T$, ou seja, faça $T \leftarrow T \cup \mathcal{S}_{\mathfrak{u}}$.

9: Seja $t$ um vértice tal que $t \notin V_{G}$.

10: Remova $S_{u}$ de $G$ e adicione $t$ a $R$, isto é, $G \leftarrow G-\mathcal{S}_{u}+t$ e $R \leftarrow R \backslash R_{u}+t$.

11: Para todo $v$ em $G-\mathcal{S}_{\mathfrak{u}}$, faça $c(v t)=c(v u)$.

12: Se ainda há mais de um terminal em $G$, vá para a linha 2.

13: Devolva T e pare.

A complexidade de tempo do Algoritmo CustoMédio é polinomial no tamanho da instância de entrada, dominada pela linha 2 onde deve-se determinar uma estrela de terminais de custo médio mínimo na instância.

Mostramos a seguir que o ALGORITMO CUSTOMÉDIO é uma 8/5-aproximação para o problema TST $T_{1,2}$. Primeiro, mostramos no lema 3.2 que o ALGORITMO CUSTOMÉdio é uma 3/2aproximação para o problema $\mathrm{TST}_{1,2}$ se a instância $\mathrm{G}, \mathrm{c}$ e R contém uma $\mathcal{X}_{\mathrm{k}}$-estrela, com $k \geqslant 6$. Caso contrário, se a instância não contém uma $\mathcal{X}_{\mathrm{k}}$-estrela, com $k \geqslant 6$, então mostramos que o ALGORITMO CUSTOMÉDIO é uma 8/5-aproximação para o problema TST 1,2 .

LEMA 3.2 Sejam G, ce R uma instância do problema $\mathrm{TST}_{1,2}$ e seja T uma árvore de Steiner de terminais folhas devolvida pelo Algoritmo CustoMédio. Se G contém uma $\mathcal{X}_{k}$-estrela com $\mathrm{k} \geqslant 6$ então

$$
c(T) \leqslant \frac{3}{2} \operatorname{opt}\left(\operatorname{TST}_{1,2}(G, c, R)\right) .
$$

Prova. Por indução em $|R|$. Se $|R|=6$ então $\mathrm{G}$ contém uma $\mathcal{X}_{6}$-estrela e temos que

$$
c(T)=\operatorname{opt}\left(\operatorname{TST}_{1,2}(G, c, R)\right) \leqslant \frac{3}{2} \operatorname{opt}\left(\operatorname{TST}_{1,2}(G, c, R)\right) .
$$

Suponha que $|R|>6$. Tome uma $\mathcal{X}_{k}$-estrela em $\mathrm{G}$ tal que $\mathrm{k}$ é máximo. Remova $\mathcal{X}_{\mathrm{k}}$ de $G$ e seja $T^{\prime}$ a árvore devolvida pelo ALGORITMO CuSTOMÉdio para a instância $G-\mathcal{X}_{k}, \mathrm{c}$ e $R \backslash R_{u}+t$, onde $u$ é o centro da $\mathcal{X}_{k}$-estrela. Então, se $T$ é a árvore devolvida pelo ALGORITMO CustoMédio para a instância $G, c$ e R, é fácil ver que

$$
c(T) \leqslant c\left(T^{\prime}\right)+k+2
$$


Por outro lado, sabemos que

$$
\operatorname{opt}\left(\operatorname{TST}_{1,2}(\mathrm{G}, \mathrm{c}, \mathrm{R})\right) \geqslant \operatorname{opt}\left(\mathrm{TST}_{1,2}\left(\mathrm{G}-\mathcal{X}_{\mathrm{k}}, \mathrm{c}, \mathrm{R} \backslash \mathrm{R}_{\mathrm{u}}+\mathrm{t}\right)\right)+\mathrm{k} .
$$

Portanto,

$$
\begin{aligned}
c(T) & \leqslant c\left(T^{\prime}\right)+k+2 \\
& \leqslant \frac{3}{2} \operatorname{opt}\left(\operatorname{TST}_{1,2}\left(G-\mathcal{X}_{k}, c, R \backslash R_{u}+t\right)\right)+k+2 \\
& \leqslant \frac{3}{2}\left(\operatorname{opt}\left(\operatorname{TST}_{1,2}(G, c, R)\right)-k\right)+k+2 \\
& =\frac{3}{2} \operatorname{opt}\left(\operatorname{TST}_{1,2}(G, c, R)\right)-\frac{k}{2}+2 \\
& \leqslant \frac{3}{2} \operatorname{opt}\left(\operatorname{TST}_{1,2}(G, c, R)\right),
\end{aligned}
$$

se $k \geqslant 6$.

Pelo lema 3.2, se uma instância $G$, c e R do problema TST $_{1,2}$ contém uma $\mathcal{X}_{k}$-estrela, com $k \geqslant 6$, então o ALGORITMO CUSTOMÉDIO sempre devolve uma árvore de Steiner de terminais folhas $T$ tal que

$$
c(T) \leqslant \frac{3}{2} \operatorname{opt}\left(\operatorname{TST}_{1,2}(\mathrm{G}, \mathrm{c}, \mathrm{R})\right) \text {. }
$$

A seguir, vamos supor que a instância $G, c$ e R não contém $\mathcal{X}_{k}$-estrelas, para $k \geqslant 6$, e mostraremos que a árvore T construída pelo ALGORITMO CUSTOMÉdio é tal que

$$
c(T) \leqslant \frac{8}{5} \operatorname{opt}\left(\mathrm{TST}_{1,2}(\mathrm{G}, \mathrm{c}, \mathrm{R})\right)
$$

Dados $G, c$ e $R$, dizemos que um vértice $v$ em $V_{G}$ domina ele próprio e os vértices adjacentes a $v$ por arestas de custo 1 . Então, o conjunto $D$ em $V_{G}$ é um conjunto dominante de $R$ em $G$ se todo terminal em $R$ é dominado por pelo menos um vértice de $D$. Um conjunto dominante de $R$ em $G$ de cardinalidade mínima é chamado um conjunto dominante mínimo de $R$ em $G$. Veja a figura 3.3.
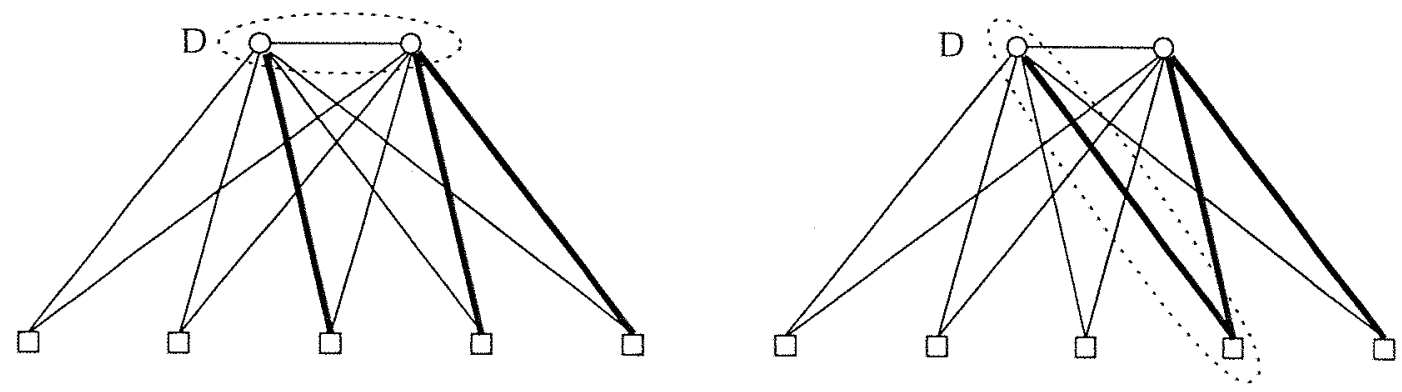

Figura 3.3: Conjuntos dominantes mínimos para duas instâncias. 
ProposiçÃo 3.3 Sejam G, c e R uma instância do problema TST $_{1,2}$ e D um conjunto dominante mínimo de R em G. Então,

$$
\operatorname{opt}\left(\mathrm{TST}_{1,2}(\mathrm{G}, \mathrm{c}, \mathrm{R})\right) \geqslant|\mathrm{R}|+|\mathrm{D}|-1 \text {. }
$$

Prova. Seja $R^{\prime} \subseteq R$ o conjunto de terminais que são dominados pelos vértices de um conjunto $D^{\prime}$, onde $D^{\prime}$ em $V_{G} \backslash R$ é o conjunto de vértices de Steiner de uma árvore de Steiner de terminais folhas ótima para $G, c$ e R. Veja a figura 3.4.

$D^{\prime}$

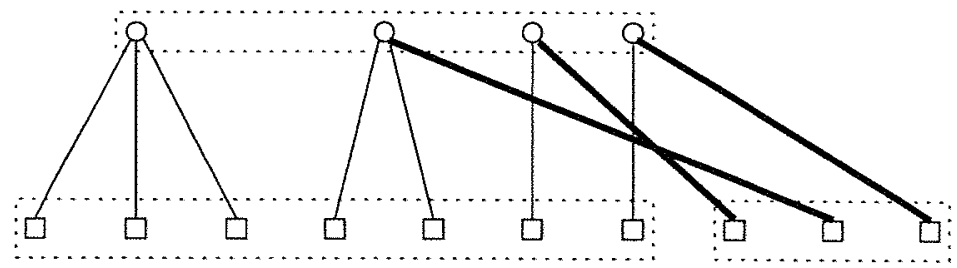

$\mathrm{R}^{\prime}$

$R \backslash R^{\prime}$

Figura 3.4: Conjunto dominante $D^{\prime} \cup R \backslash R^{\prime}$ para uma instância $G, c$ e R.

Observe que pelo menos $\left|D^{\prime}\right|-1$ arestas são necessárias para conectar os vértices de $D^{\prime}$ em uma árvore. Assim,

$$
\begin{aligned}
\operatorname{opt}\left(\operatorname{TST}_{1,2}(G, c, R)\right) & \geqslant\left|R^{\prime}\right|+\left|D^{\prime}\right|-1+2\left|R \backslash R^{\prime}\right| \\
& =|R|+\left|D^{\prime}\right|+\left|R \backslash R^{\prime}\right|-1 .
\end{aligned}
$$

Como $D^{\prime} \cup\left(R \backslash R^{\prime}\right)$ é um conjunto dominante de $R$ em G e $D^{\prime}$ e $R \backslash R^{\prime}$ são disjuntos, vale que $|D| \leqslant\left|D^{\prime} \cup\left(R \backslash R^{\prime}\right)\right|=\left|D^{\prime}\right|+\left|R \backslash R^{\prime}\right|$. Assim,

$$
\begin{aligned}
\operatorname{opt}\left(\operatorname{TST}_{1,2}(G, c, R)\right) & \geqslant|R|+\left|D^{\prime}\right|+\left|R \backslash R^{\prime}\right|-1 \\
& \geqslant|R|+|D|-1 .
\end{aligned}
$$

Seja $D$ um conjunto dominante mínimo de $R$ em $G$. Podemos particionar $R$ em relação a $D$ da seguinte forma. Atribua cada terminal $r$ em $R$ a um conjunto representando um elemento de $\mathrm{D}$ que o domina. Se dois ou mais vértices de $\mathrm{D}$ dominam o vértice $r$, atribua $r$ arbitrariamente a um dos conjuntos. A figura 3.5 mostra um exemplo dessa definição. Sejam $\mathscr{D}_{1}, \ldots, \mathscr{D}_{\mathrm{q}}$ as partições de $R$ consistindo de exatamente 5 terminais, com $0 \leqslant q \leqslant\lfloor n / 5\rfloor$.

PROPOSIÇÃO 3.4 Sejam G, c e R uma instância do problema TST 1,2 , D um conjunto dominante mínimo de $\mathrm{R}$ em $\mathrm{G}$ e q o número de conjuntos em $\mathrm{D}$ que contêm 5 terminais. Então,

$$
\operatorname{opt}\left(\operatorname{TST}_{1,2}(G, c, R)\right) \geqslant \frac{5}{4}|R|-\frac{q}{4}-1
$$

Prova. De acordo com a partição de $R$ em relação a $D$, temos que

$$
5 q+4(|D|-q) \geqslant|R|
$$




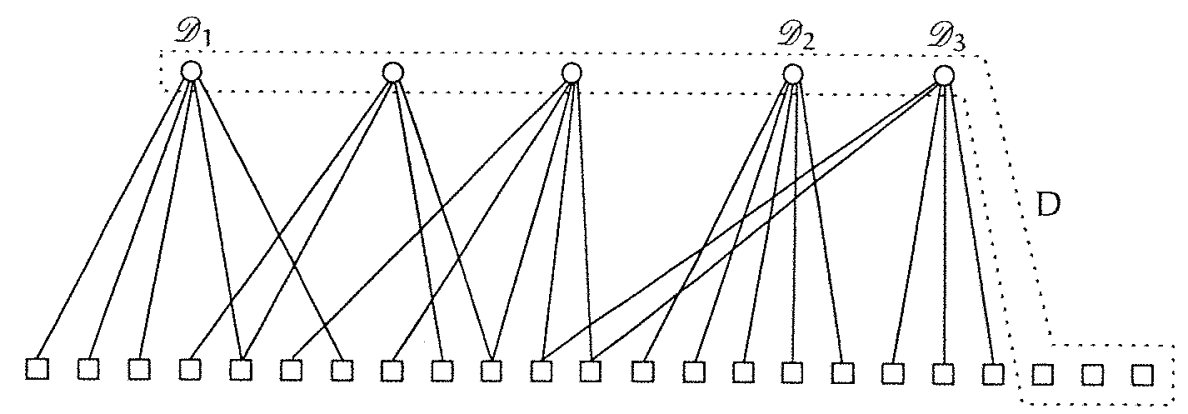

Figura 3.5: Um exemplo de particionamento de um conjunto dominante mínimo D com $q=3$.

e então

$$
|D| \geqslant \frac{|R|-q}{4}
$$

Logo,

$$
\begin{aligned}
\operatorname{opt}\left(\operatorname{TST}_{1,2}(G, c, R)\right) & \geqslant|R|+|D|-1 \\
& \geqslant|R|+\frac{|R|-q}{4}-1 \\
& =\frac{5}{4}|R|-\frac{q}{4}-1
\end{aligned}
$$

onde a desigualdade (3.1) vale pela proposição 3.3 .

Agora, podemos mostrar o seguinte resultado.

LEMA 3.5 Sejam G, c e R uma instância do problema $\mathrm{TST}_{1,2}$ e T uma árvore de Steiner de terminais folhas devolvida pelo ALGORITMO CUSTOMÉDIo para essa instância. Se G não contém uma $\mathcal{X}_{\mathrm{k}}$-estrela para $k \geqslant 6$ então

$$
c(T) \leqslant \frac{8}{5} \operatorname{opt}\left(\operatorname{TST}_{1,2}(\mathrm{G}, \mathrm{c}, \mathrm{R})\right) \text {. }
$$

Prova. Suponha que o ALGORITMO Customédio reduz totalmente $j \mathcal{X}_{k_{i}}$-estrelas, com $1 \leqslant$ $i \leqslant j$ e $k_{i} \geqslant 2$, quando operando sobre uma instância $G, c$ e $R$ do problema $T_{S T}$, . Observe que apesar de uma instância não conter uma $\mathcal{X}_{\mathrm{k}}$-estrela com $k \geqslant 6$, a $\mathcal{X}_{\mathrm{k}_{\mathrm{i}}}$-estrela reduzida pode ser uma $\mathcal{X}_{6}$-estrela, para algum $i \geqslant 2$. Entretanto, observe também que é impossível que uma $\mathcal{X}_{k_{i}}$-estrela seja uma $\mathcal{X}_{k}$-estrela com $k \geqslant 7$, para algum $i$.

Note que a $\mathcal{X}_{k_{i}}$-estrela é uma subárvore da árvore de Steiner de terminais folhas T devolvida pelo ALGORITMO CUSTOMÉdio e que seu custo é $k_{i}$. Como a redução da $\mathcal{X}_{k_{i}}$-estrela transforma $k_{i}$ terminais em um novo terminal, o número de terminais é decrementado de $k_{i}-1$ nesse processo. Após reduzir a $\mathcal{X}_{k_{j}}$-estrela, o número de terminais restantes na instância de entrada é $|R|-\sum_{i=1}^{j}\left(k_{i}-1\right)$. Para reduzir esses terminais restantes, o ALGORITMO CUSTOMÉdIo produz 
uma estrela de terminais com custo menor ou igual a $2\left(|R|-\sum_{i=1}^{j}\left(k_{i}-1\right)\right)$. Assim, o custo total de Té tal que

$$
c(T) \leqslant \sum_{i=1}^{j} k_{i}+2\left(|R|-\sum_{i=1}^{j}\left(k_{i}-1\right)\right)=2|R|-\sum_{i=1}^{j}\left(k_{i}-2\right) .
$$

Ou seja, $c(T) \leqslant 2|R|-p$, onde $p=\sum_{i=1}^{j}\left(k_{i}-2\right)$.

Lembre-se que particionamos $R$ em conjuntos disjuntos e que $\mathscr{D}_{1}, \ldots, \mathscr{D}_{\mathrm{q}}, \operatorname{com} 0 \leqslant \mathrm{q} \leqslant$ $\lfloor\{R \mid / 5\rfloor$, são os conjuntos consistindo de exatamente 5 terminais. Em outras palavras, existem q $\mathcal{X}_{5}$-estrelas disjuntas na instância $G, c$ e R. Observe que uma estrela de terminais escolhida pelo ALGORITMO CUSTOMÉdio que não corresponde a qualquer conjunto $\mathscr{D}_{1}, \ldots, \mathscr{D}_{\mathrm{q}}$ pode 'estragar' no máximo dois desses conjuntos, como mostra a figura 3.6.

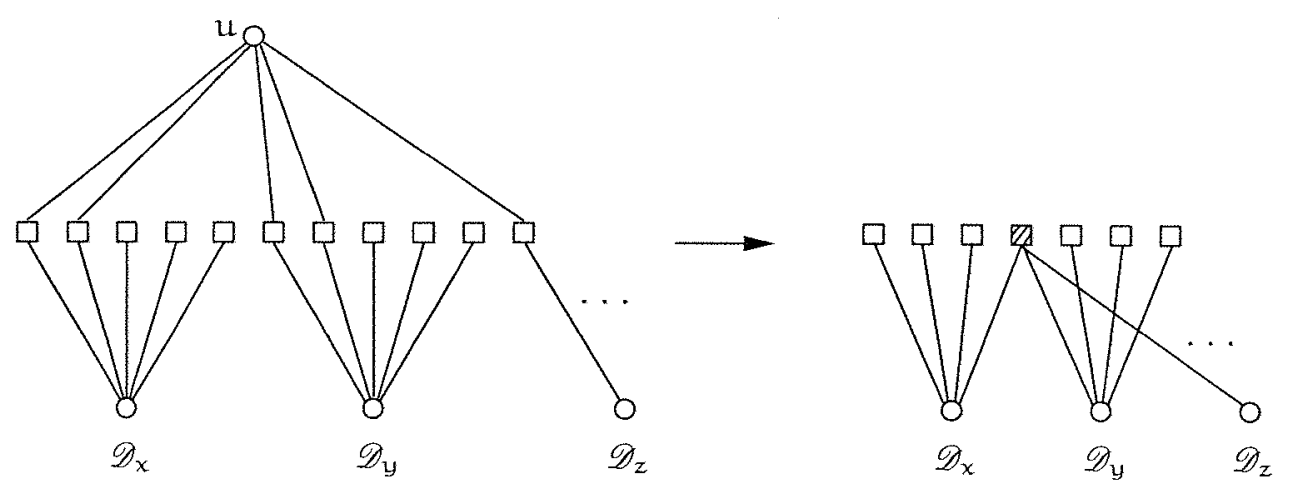

Figura 3.6: Um exemplo de uma $\mathcal{X}_{5}$-estrela tomada pelo algoritmo, com centro não pertencente a $D$ e a consequêencia de sua redução. $O$ vértice $u$ é o vértice de Steiner centro de uma $\mathcal{X}_{5}$-estrela escolhida pelo algoritmo e o vértice hachureado é o vértice terminal resultado da redução da $\mathcal{X}_{5}$-estrela.

Dessa forma, temos que $j \geqslant q / 2 e$

$$
p=\sum_{i=1}^{j}\left(k_{i}-2\right) \geqslant \sum_{i=1}^{q / 2}(5-2)=\frac{3}{2} q
$$

Então, se $T^{*}$ é uma árvore de Steiner de terminais folhas ótima para a instância $G, c$ e R, temos que

$$
\begin{aligned}
\frac{c(T)}{c\left(T^{*}\right)} & \leqslant \frac{2|R|-3 q / 2}{5|R| / 4-q / 4-1} \\
& =\frac{8|R|-6 q}{5|R|-q-4} \\
& \leqslant \frac{8}{5},
\end{aligned}
$$


se $q \geqslant 2$. Como $0 \leqslant q \leqslant\lfloor|R| / 5\rfloor$, então

$$
\frac{c(T)}{c\left(T^{*}\right)} \leqslant \frac{8}{5}
$$

se $|R| \geqslant 10$. Observe finalmente que se $|R|<10$, uma solução ótima pode ser obtida por busca exaustiva em tempo constante.

Finalmente, podemos mostrar que o ALGORITMO CUSTOMÉdio é uma 8/5-aproximação para o problema $\mathrm{TST}_{1,2}$.

TEOREMA 3.6 O ALGORITMO CUSTOMÉDIO é uma 8/5-aproximação para o problema TST $_{1,2}$.

Prova. Segue imediatamente dos lemas 3.2 e 3.5 .

\subsection{Uma 17/12-aproximação para o problema $\operatorname{TST}_{1,2}$}

A seguir, apresentamos um algoritmo de aproximação que recebe uma instância $G, c$ e $R$ do problema $\mathrm{TST}_{1,2}$ e constrói uma árvore de Steiner de terminais folhas $\mathrm{T}$ tal que

$$
c(T) \leqslant \frac{17}{12} \operatorname{opt}\left(\operatorname{TST}_{1,2}(\mathrm{G}, \mathrm{c}, \mathrm{R})\right) \text {. }
$$

Por toda esta seção consideramos a instância de entrada viável e $|R| \geqslant 3$. O algoritmo de aproximação que propomos constrói uma árvore $T_{u}$ para cada $u$ em $V_{G} \backslash R$. Cada árvore $T_{u}$ é tal que $c\left(T_{u}\right)$ é no máximo 17/12 vezes maior que o custo de qualquer árvore de Steiner de terminais folhas usando $u$ como um vértice de Steiner. A árvore devolvida pelo algoritmo é uma árvore de custo mínimo dentre todas as árvores $T_{u}$, para todo $u$ em $V_{G} \backslash R$. Como uma árvore de Steiner de terminais folhas de custo mínimo deve usar pelo menos um vértice de Steiner da instância, a árvore devolvida tem custo no máximo $17 / 12$ vezes maior que o custo de uma árvore de Steiner de terminais folhas de custo ótimo para essa instância.

O ALGORITMO TERMINAL ${ }_{1,2}$, que descrevemos na seção 3.3.3, escolhe inicialmente um vértice de Steiner $u$ em $V_{G} \backslash R$ e executa duas reduções na instância $G, c, R$ e $u$. Na primeira delas, os terminais que são conectados ao vértice $u$ por arestas de custo 1 são removidos. $\mathrm{Na}$ segunda, vértices de Steiner ligados a u e conectados a pelos menos cinco terminais por arestas de custo 1 também são eliminados. Essas reduções são detalhadas na seção 3.3.1. Em seguida, o algoritmo constrói uma árvore de Steiner de terminais folhas para a instância que resta após essas reduções. A idéia nessa construção é realizar um empacotamento dos terminais, como veremos na seção 3.3.2. No final, os terminais removidos nas reduções são de certa forma conectados ao vértice $u$. $O$ algoritmo executa essa sequiência de passos para cada vértice de Steiner $u$ da instância de entrada e devolve a árvore de Steiner de terminais folhas de menor custo como resultado. 
Então, mostramos o seguinte resultado.

TEOREMA 3.7 Existe um algoritmo de aproximação de tempo polinomial para o problema $\mathrm{TST}_{1,2}$ com razão de aproximação 17/12.

\subsubsection{Reduções}

O ALGORITMO EMPACOTA é o núcleo do ALGORITMO TERMINAL ${ }_{1,2}$. Antes de apresentálo, descrevemos duas reduções usadas para remover alguns vértices de $R$, que descrevemos a seguir. Os lemas abaixo mostram como obter a árvore de Steiner de terminais folhas para o conjunto $R$ mantendo a razão de aproximação obtida pelo ALGORITMO EMPACOTA.

A idéia da REDUÇão 1 é que se consideramos que um vértice de Steiner u é parte de uma árvore de Steiner de terminais folhas construída pelo ALGORITMO TERMINAL1,2, então toda aresta de custo 1 conectando o vértice $u$ a um vértice terminal pode fazer parte de uma árvore de Steiner de terminais folhas de custo mínimo. A figura 3.7 dá uma idéia da REDUÇÃo 1. Observe ainda que o fator $17 / 12$ aparecendo na sentença do lema 3.8 pode ser substituído por qualquer valor maior ou igual a 1.

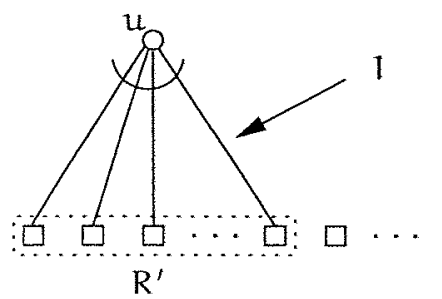

Figura 3.7: $O$ conjunto de terminais $R^{\prime}$ ligados ao vértice $u$ por arestas de custo 1 devolvido pela REDUÇÃO 1.

REDUÇÃo 1: recebe um grafo completo $G$, uma função custo $c: E_{G} \rightarrow\{1,2\}$, um conjunto $R \subseteq V_{G}$ de terminais e um vértice $u$ em $V_{G} \backslash R$ e devolve um conjunto $R^{\prime} \subseteq R$.

1: Seja $R^{\prime}$ o conjunto $\{r: r \in R$ e $c(r u)=1\}$.

2: Devolva $R^{\prime}$ e pare.

Seja $G, c$ e $R$ uma instância do problema $T_{S T} T_{1,2}$ e seja $u$ um vértice em $V_{G} \backslash R$. Denotamos por opt $\left(\mathrm{TST}_{1,2}(G, c, R, u)\right)$ o custo de uma árvore de Steiner de terminais folhas ótima contendo o vértice $u$ para a instância $G, c$ e R.

LEMA 3.8 Dado o conjunto $R^{\prime}$ construído pela REDUÇÃo 1 e uma árvore de Steiner de terminais folhas $\mathrm{T}_{1}$ contendo u para a instancia $\mathrm{G}, \mathrm{c}$ e $\mathrm{R} \backslash \mathrm{R}^{\prime}$ tal que

$$
c\left(T_{1}\right) \leqslant \frac{17}{12} \text { opt }\left(\operatorname{TST}_{1,2}\left(G, c, R \backslash R^{\prime}, u\right)\right),
$$


podemos encontrar em tempo polinomial uma árvore de Steiner de terminais folhas $\mathrm{T}_{2}$ contendo u para a instância $\mathrm{G}, \mathrm{c}$ e R tal que

$$
c\left(T_{2}\right) \leqslant \frac{17}{12} \operatorname{opt}\left(\operatorname{TST}_{1,2}(G, c, R, u)\right)
$$

Prova. Como a remoção de um terminal de $R$ decrementa o custo da árvore de Steiner de terminais folhas de pelo menos 1 , temos que

$$
\operatorname{opt}\left(\operatorname{TST}_{1,2}\left(G, c, R \backslash R^{\prime}, u\right)\right) \leqslant \operatorname{opt}\left(\operatorname{TST}_{1,2}(G, c, R, u)\right)-\left|R^{\prime}\right| .
$$

Construímos $T_{2}$ a partir de $T_{1}$ adicionando a $T_{1}$ alguns vértices e arestas. Adicionamos a $T_{1}$ os vértices em $R^{\prime}$ e as arestas conectando os vértices de $R^{\prime}$ ao vértice $u$. Como cada uma dessas arestas tem custo 1 , temos que

$$
c\left(T_{2}\right)=c\left(T_{1}\right)+\left|R^{\prime}\right|
$$

Usando a hipótese (3.2), combinando a desigualdade (3.3) e a igualdade (3.4), obtemos

$$
\begin{aligned}
c\left(T_{2}\right) & =c\left(T_{1}\right)+\left|R^{\prime}\right| \\
& \leqslant \frac{17}{12} \operatorname{opt}\left(\operatorname{TST}_{1,2}\left(G, c, R \backslash R^{\prime}, u\right)\right)+\left|R^{\prime}\right| \\
& \leqslant \frac{17}{12}\left(\operatorname{opt}\left(\operatorname{TST}_{1,2}(G, c, R, u)\right)-\left|R^{\prime}\right|\right)+\left|R^{\prime}\right| \\
& \leqslant \frac{17}{12} \operatorname{opt}\left(\operatorname{TST}_{1,2}(G, c, R, u)\right) .
\end{aligned}
$$

A idéia da REDUÇ̃̃ 2 é que um vértice de Steiner conectado a 'vários' terminais por arestas de custo 1 é um vértice adequado para fazer parte de uma árvore de Steiner de terminais folhas. Neste caso, vários significa pelo menos 5. Veja a figura 3.8. Uma observação importante é que o trabalho do ALGORITMO EMPACOTA, que apresentamos na próxima seção, é facilitado se trocamos 5 por 4 na REDUÇÃo 2 . No entanto, em se fazendo essa troca, essa redução levaria a uma razão de aproximação de $3 / 2$ ao invés de $17 / 12$, e como $3 / 2>17 / 12$, o resultado principal para o problema $\operatorname{TST}_{1,2}$ não valeria.

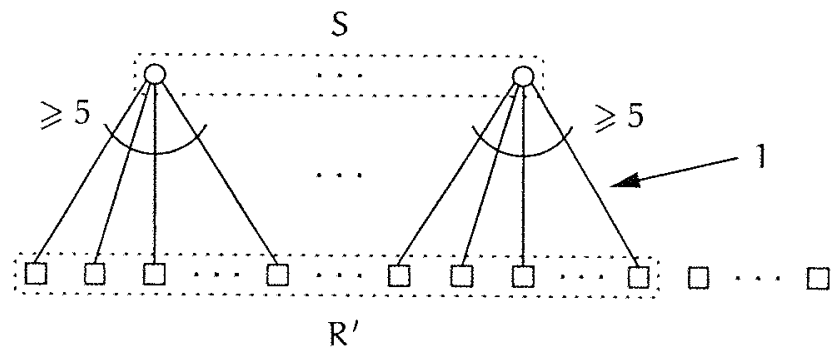

Figura 3.8: O conjunto de terminais $R^{\prime}$ ligados a vértices de Steiner do conjunto $S$ por arestas de custo 1, ambos os conjuntos devolvidos pela REDUÇÃO 2. 
REDUÇ̃̃o 2: recebe um grafo completo $G$, uma função custo $c: E_{G} \rightarrow\{1,2\}$, um conjunto $R \subseteq V_{G}$ de terminais e devolve os conjuntos $R^{\prime} \subseteq R$ e $S \in V_{G} \backslash R$.

1: $\mathrm{R}^{\prime}:=\emptyset$

2: $S:=\emptyset$

3: Para cada $s$ em $V_{G} \backslash R$, seja $N(s):=\{r: r \in R$ e $c(r s)=1\}$.

4: Se existe $s$ em $V_{G} \backslash R$ tal que $|N(s)| \geqslant 5$, então faça $R^{\prime}:=R^{\prime} \cup N(s), S:=S \cup\{s\}, R:=R \backslash N(s)$ e volte para a linha 3 .

5: Devolva $\left(R^{\prime}, S\right)$ e pare.

A REDUÇÃO 2 reúne todos os vértices de Steiner que estão conectados a pelo menos cinco terminais por arestas de custo $1 \mathrm{em}$ um conjunto $S$ e todos esses terminais em um conjunto $\mathrm{R}^{\prime}$.

Observe agora que a razão $17 / 12$ aparecendo na sentença do lema 3.9 pode ser trocada por qualquer valor maior ou igual a $7 / 5$.

LEMA 3.9 Dados os conjuntos $\mathrm{R}^{\prime}$ e $\mathrm{S}$ construídos pela REDUÇão 2 e uma árvore de Steiner de terminais folhas $T_{1}$ contendo um vértice de Steiner $u$ em $V_{G} \backslash R$ para a instância $G, c e R \backslash R^{\prime}$ tal que

$$
c\left(T_{1}\right) \leqslant \frac{17}{12} \operatorname{opt}\left(\operatorname{TST}_{1,2}\left(G, c, R \backslash R^{\prime}, u\right)\right),
$$

podemos encontrar em tempo polinomial uma árvore de Steiner de terminais folhas $\mathrm{T}_{2}$ contendo o vértice u para a instância $\mathrm{G}, \mathrm{c}$ e R tal que

$$
c\left(T_{2}\right) \leqslant \frac{17}{12} \operatorname{opt}\left(\operatorname{TST}_{1,2}(G, c, R, u)\right)
$$

PROVA. Como a remoção de qualquer vértice terminal em $R$ decrementa o custo da árvore de Steiner de terminais folhas ótima de pelo menos 1 , temos que

$$
\operatorname{opt}\left(\operatorname{TST}_{1,2}\left(G, c, R \backslash R^{\prime}, u\right)\right) \leqslant \operatorname{opt}\left(\operatorname{TST}_{1,2}(G, c, R, u)\right)-\left|R^{\prime}\right| .
$$

Construímos $T_{2}$ a partir de $T_{1}$ pela adição de alguns vértices e arestas à $T_{1}$. Adicionamos a $T_{1}$ os vértices em $R^{\prime} \cup S$. Adicionamos também as arestas de custo 1 conectando vértices em $S$ a vértices em $R^{\prime}$. Se necessário, para cada vértice $s$ em $S$ adicionamos a aresta conectando $s$ ao vértice $u$. Então, é fácil ver que $T_{2}$ é uma árvore de Steiner de terminais folhas contendo o vértice u para a instância $G, c$ e R. O custo de $T_{2}$ é então

$$
c\left(T_{2}\right) \leqslant c\left(T_{1}\right)+\left|R^{\prime}\right|+2|S|
$$

Por construção temos que para cada vértice em $S$ existem pelo menos 5 vértices em $R^{\prime}$. Assim, sabemos que

$$
5|S| \leqslant\left|R^{\prime}\right|
$$

Combinando as duas últimas desigualdades, temos que

$$
c\left(T_{2}\right) \leqslant c\left(T_{1}\right)+\left|R^{\prime}\right|+2|S| \leqslant c\left(T_{1}\right)+\left|R^{\prime}\right|+\frac{2}{5}\left|R^{\prime}\right| \leqslant c\left(T_{1}\right)+\frac{7}{5}\left|R^{\prime}\right| .
$$


Usando a hipótese (3.5) e as desigualdades (3.6) e (3.7), obtemos que

$$
\begin{aligned}
c\left(T_{2}\right) & \leqslant c\left(T_{1}\right)+\frac{7}{5}\left|R^{\prime}\right| \\
& \leqslant \frac{17}{12} \operatorname{opt}\left(\operatorname{TST}_{1,2}\left(G, c, R \backslash R^{\prime}, u\right)\right)+\frac{7}{5}\left|R^{\prime}\right| \\
& \leqslant \frac{17}{12}\left(\operatorname{opt}\left(\operatorname{TST}_{1,2}(G, c, R, u)\right)-\left|R^{\prime}\right|\right)+\frac{7}{5}\left|R^{\prime}\right| \\
& \leqslant \frac{17}{12} \operatorname{opt}\left(\operatorname{TST}_{1,2}(G, c, R, u)\right) .
\end{aligned}
$$

\subsubsection{Algoritmo Empacota}

Similarmente à REDUÇÃo 2, o ALGORITMO EMPACOTA tenta utilizar vértices de Steiner conectados a 'muitos' vértices terminais por arestas de custo 1 . Nesta caso, muitos significa 4 ou 3. Comparamos o número de vértices de Steiner que podemos encontrar com o número de vértices de Steiner que uma árvore de Steiner de terminais folhas pode utilizar. Essa comparação nos conduz ao problema conhecido como problema de empacotamento de conjuntos, conforme descrevemos a seguir.

Seja $\mathcal{C}$ uma coleção de subconjuntos de um conjunto finito $\mathcal{U}$. Um empacotamento de conjuntos é uma coleção de subconjuntos mutuamente disjuntos em $\mathcal{C}$. A figura 3.9 mostra um exemplo de um empacotamento. O problema de encontrar a maior coleção de subconjuntos de tamanho $\mathrm{k}$ mutuamente disjuntos em uma dada coleção $\mathcal{C}$ de subconjuntos de um conjunto $\mathcal{U}$ é chamado de problema do empacotamento de conjuntos, onde $k$ é um parâmetro de entrada. Esse problema é descrito formalmente a seguir.

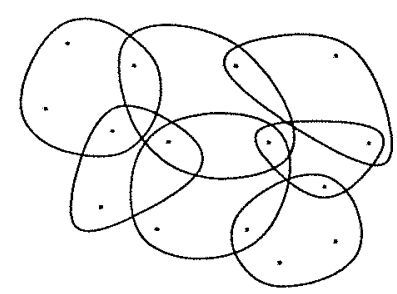

(a)

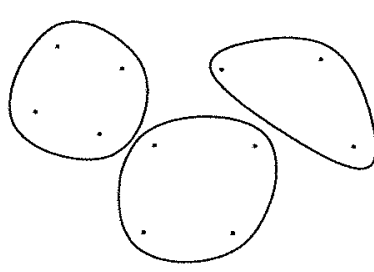

(b)

Figura 3.9: (a) Uma coleção de subconjuntos $\mathcal{C}$ e (b) um empacotamento de $\mathcal{C}$ de cardinalidade máxima.

Problema $\mathrm{EC}(\mathcal{C}, \mathrm{k})$ : dada uma coleção $\mathcal{C}$ de subconjuntos de tamanho $\mathrm{k}$ de um conjunto finito $\mathcal{U}$, encontrar uma coleção de conjuntos mutuamente disjuntos em $\mathcal{C}$ de cardinalidade máxima. 
O problema EC é APX-difícil (Papadimitriou, 1994). Como apresentamos em seguida, o ALGORITMO EMPACOTA realiza um empacotamento de conjuntos de tamanho 4 . Hurkens \& Schrijver (1989) propuseram um algoritmo de aproximação de tempo polinomial para o problema EC com razão de aproximação $1 /(2+\varepsilon)$, para $k=4$ e $\varepsilon$ fixo.

O ALGORITMO EMPACOTA constrói uma coleção $\mathcal{C}$ de subconjuntos de 3 ou 4 terminais em $R$ que são conectados a vértices de Steiner da instância $G, c$ e $R$ por arestas de custo 1, após as reduçōes da seção anterior terem sido realizadas sobre essa instância. O algoritmo constrói então um empacotamento $\mathcal{A}$ dos conjuntos de tamanho $4 \mathrm{em} \mathcal{C}$ usando o algoritmo de Hurkens $\&$ Schrijver (1989) e, em seguida, adiciona ao empacotamento $\mathcal{A}$ os conjuntos de tamanho 3 de $\mathcal{C}$ de forma gulosa. No final, o algoritmo constrói uma árvore a partir de $\mathcal{A}$ e dos terminais restantes não empacotados em $\mathcal{A}$.

ALGORITMO EMPACOTA: recebe um grafo completo $G$, uma função custo $c$ : $E_{G} \rightarrow\{1,2\}$, um conjunto $R \subseteq V_{G}$ de terminais e um vértice $u$ em $V_{G} \backslash R$, e devolve uma árvore de Steiner de terminais folhas.

1: Para cada s em $V_{G} \backslash R$ seja $N(s)$ o conjunto $\{r: r \in R$ e $c(r s)=1\}$.

2: Seja $\mathcal{C}$ a coleção de conjuntos $\left\{N(s): s \in V_{G} \backslash R\right\} \cup\{C: C \subseteq N(s)$ para algum $s, e|C|=3\}$.

3: Construa um empacotamento de conjuntos $\mathcal{A}$ de $\mathcal{C}$ usando o lema 3.10 .

4: Para cada $A \in \mathcal{A}$, escolha um único vértice $v_{\mathcal{A}}$ em $V_{G} \backslash R$ tal que $A \subset N\left(v_{A}\right)$.

5: Seja $V_{\mathrm{T}}$ o conjunto de vértices $\{u\} \cup\{R\} \cup\left\{v_{\mathcal{A}}: A \in \mathcal{A}\right\}$.

6: Seja $E_{\mathrm{T}}$ o conjunto de arestas $\left\{\mathrm{rs}: \mathrm{r}\right.$ é coberto por $\mathcal{A}$ e $\mathrm{s}=\nu_{\mathrm{A}}$ para algum $\left.A \in \mathcal{A}\right\} \cup\{\mathrm{su}: \mathrm{s}=$ $v_{A}$ para algum $\left.A \in \mathcal{A}\right\} \cup\{$ ru: r não é coberto por $\mathcal{A}\}$.

7: Devolva $T:=\left(V_{T}, E_{T}\right)$ e pare.

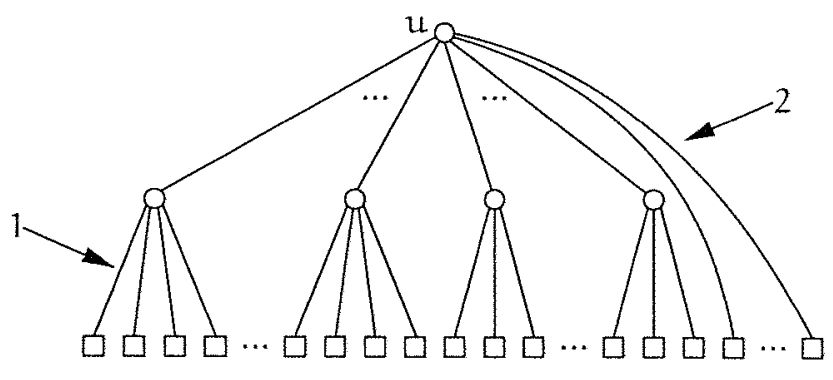

Figura 3.10: Resultado da execução do ALGORITMO EMPACOTA.

LEMA 3.10 Seja $\mathcal{C}$ uma coleção de subconjuntos de um conjunto finito $\mathcal{U}$ tal que cada conjunto em $\mathcal{C}$ tem 3 ou 4 elementos e tal que se o conjunto $\mathrm{C}$ em $\mathcal{C}$ tem 4 elementos, então cada 3 -subconjunto de $\mathrm{C}$ também pertence a $\mathcal{C}$. Podemos encontrar em tempo polinomial um empacotamento de conjuntos $\mathcal{A}$ de $\mathcal{C}$ com $a_{4}$ conjuntos de cardinalidade 4 e $a_{3}$ conjuntos de cardinalidade 3 tal que

$$
\frac{8 b_{4}+6 b_{3}-2 a_{4}-a_{3}}{5 b_{4}+4 b_{3}} \leqslant \frac{17}{12}
$$

onde $\mathrm{b}_{4}$ e $\mathrm{b}_{3}$ são o número de conjuntos de cardinalidade 4 e 3 , respectivamente, em um empacotamento de conjuntos arbitrário não vazio $\mathcal{B}$ de $\mathcal{C}$. 
PROVA. Começamos empacotando conjuntos de tamanho 4. Usamos então o algoritmo de Hurkens \& Schrijver (1989) que garante que, para qualquer $\varepsilon$ fixo, podemos encontrar em tempo polinomial um empacotamento com pelo menos $b_{4} /(2+\varepsilon)$ conjuntos. Precisamos de um empacotamento com $a_{4}$ conjuntos de tamanho 4 , onde

$$
a_{4} \geqslant \frac{3}{8} b_{4}
$$

e assim fazemos $\varepsilon=2 / 3$. Além desses $a_{4}$ conjuntos, podemos adicionar a $\mathcal{A}$ quantos conjuntos de 3 elementos são possíveis, de maneira gulosa.

Sejam $\mathcal{B}_{4}$ e $\mathcal{B}_{3}$ a coleção de conjuntos de tamanho 4 e 3 , respectivamente, no empacotamento $\mathcal{B}$. Como uma conseqüência da escolha gulosa dos conjuntos de tamanho $3 \mathrm{em} \mathcal{A}$, podemos observar que cada conjunto $\mathrm{B}$ em $\mathcal{B}_{4}$ tem pelo menos 2 elementos cobertos por $\mathcal{A}$, já que, caso contrário, um 3-subconjunto de $\mathrm{B}$ seria adicionado a $\mathcal{A}$. Também, cada conjunto $\mathrm{B}$ em $\mathcal{B}_{3}$ tem pelo menos 1 elemento coberto por $\mathcal{A}$, pois, caso contrário, B seria adicionado a $\mathcal{A}$. Assim, o número total de elementos cobertos por $\mathcal{A}$ é pelo menos $2 \mathrm{~b}_{4}+\mathrm{b}_{3}$. Por outro lado, $\mathcal{A}$ pode cobrir no máximo $4 a_{4}+3 a_{3}$ elementos.

Portanto, segue que

$$
4 a_{4}+3 a_{3} \geqslant 2 b_{4}+b_{3}
$$

Usando a desigualdade (3.9) e a hipótese que $\mathcal{B}$ é não vazio obtemos que

$$
\begin{aligned}
\frac{8 b_{4}+6 b_{3}-2 a_{4}-a_{3}}{5 b_{4}+4 b_{3}} & =\frac{24 b_{4}+18 b_{3}-6 a_{4}-3 a_{3}}{15 b_{4}+12 b_{3}} \\
& \leqslant \frac{24 b_{4}+18 b_{3}-2 a_{4}-2 b_{4}-b_{3}}{15 b_{4}+12 b_{3}} \\
& =\frac{22 b_{4}+17 b_{3}-2 a_{4}}{15 b_{4}+12 b_{3}} .
\end{aligned}
$$

Agora, usando a desigualdade (3.8) obtemos que

$$
\begin{aligned}
\frac{22 b_{4}+17 b_{3}-2 a_{4}}{15 b_{4}+12 b_{3}} & \leqslant \frac{22 b_{4}+17 b_{3}-3 b_{4} / 4}{15 b_{4}+12 b_{3}} \\
& =\frac{85 b_{4} / 4+17 b_{3}}{15 b_{4}+12 b_{3}} \\
& \leqslant \max \left\{\frac{85 b_{4} / 4}{15 b_{4}}, \frac{17 b_{3}}{12 b_{3}}\right\} \\
& =\frac{17}{12} .
\end{aligned}
$$


LEMA 3.11 Para toda instância $G, c, R$ e u em $V_{G} \backslash R$ tal que nenhuma outra redução é possivel em $R$ usando a REDUÇÃO 1 e a REDUÇÃO 2, o ALGORITMO EMPACOTA constrói uma árvore de Steiner de terminais folhas $\mathrm{T}$ tal que

$$
c(T) \leqslant \frac{17}{12} \operatorname{opt}\left(\operatorname{TST}_{1,2}(G, c, R, u)\right) .
$$

PRova. Seja $T^{*}$ uma árvore de Steiner de terminais folhas ótima contendo o vértice $u$ para a instância $G, c$ e R. Seja $B_{4}$ o conjunto de vértices de Steiner em $T^{*}$ conectados a exatamente 4 terminais usando arestas de custo 1 . Sejam $B_{3}$ e $B_{2}$ definidos de forma similar. Sejam ainda $b_{4}, b_{3}$ $e b_{2}$ as cardinalidades dos conjuntos $B_{4}, B_{3}$ e $B_{2}$, respectivamente. No que segue, consideramos $\mathrm{T}^{*}$ como uma árvore com raiz $u$.

Seja s um vértice em $B_{4}$. Podemos associar custo 5 para s: existem 4 arestas de custo 1 conectando $s$ a vértices terminais e uma aresta conectando $s$ ao seu ancestral em $T^{*}$. Do mesmo modo, podemos associar custo 4 e 3 para os vértices de Steiner em $B_{3}$ e $B_{2}$, respectivamente. Observe que as arestas mencionadas são todas disjuntas.

Então, acabamos de considerar o custo de conectar exatamente $4 b_{4}+3 b_{3}+2 b_{2}$ vértices terminais em $\mathrm{T}^{*}$. Considere um terminal $r$ não coberto. Afirmamos que podemos atribuir custo 2 para cada um desses vértices. De fato, ou (i) r está conectado em $T^{*}$ por uma aresta de custo 2 ou (ii) $r$ está conectado a um vértice de Steiner s por uma aresta de custo 1 . Se (i) acontece então podemos atribuir custo 2 a $r$ devido à aresta que conecta $r$ à árvore. Se (ii) ocorre, observe que como a REDUÇÃO 1 foi executada, temos que $s \neq u$. Logo, $s$ tem um ancestral em $T^{*}$. E também, como a REDUÇÃO 2 foi executada, s se conecta a no máximo 4 terminais por arestas de custo 1. Mas como $r$ não é um dos $4 b_{4}+3 b_{3}+2 b_{2}$ terminais, podemos dizer que $r$ é o único terminal conectado a s por uma aresta de custo 1 . Assim, podemos atribuir custo 1 para a aresta rs e custo 1 para a aresta conectando s ao seu ancestral em $T^{*}$.

Portanto, temos que

$$
c\left(T^{*}\right) \geqslant 5 b_{4}+4 b_{3}+3 b_{2}+2 b_{1}
$$

onde $b_{1}:=|R|-\left(4 b_{4}+3 b_{3}+2 b_{2}\right)$.

Agora, considere o custo da árvore construída pelo ALGORITMO EMPACOTA. A construção é baseada no empacotamento de conjuntos $\mathcal{A}$, onde para cada $A$ em $\mathcal{A}$ corresponde um vértice de Steiner $v_{A}$.

$O$ conjunto de arestas $E_{T}$ definido no algoritmo pode ser particionado em $E_{1}, E_{2}$ e $E_{3}$, onde $E_{1}:=\left\{\right.$ rs: r é coberto por $\mathcal{A}$ e $s=v_{\mathcal{A}}$ para algum $\left.A \in \mathcal{A}\right\}, E_{2}:=\left\{\right.$ su: $s=v_{\mathcal{A}}$ para algum $\left.A \in \mathcal{A}\right\}$ e $E_{3}:=\{r u: r$ não é coberto por $\mathcal{A}\}$. Sejam $a_{4}$ e $a_{3}$ o número de conjuntos em $\mathcal{A}$ com 4 e 3 elementos, respectivamente. $O$ custo de $E_{1}$ é dado por

$$
c\left(E_{1}\right)=\left|E_{1}\right|=4 a_{4}+3 a_{3},
$$

o custo de $E_{2}$ é

$$
c\left(E_{2}\right) \leqslant 2\left|E_{2}\right|=2\left(a_{4}+a_{3}\right)
$$

e o custo de $E_{3}$ é

$$
c\left(E_{3}\right) \leqslant 2\left|E_{3}\right|=2\left(|R|-\left(4 a_{4}+3 a_{3}\right)\right) .
$$


Assim, temos que

$$
\begin{aligned}
c(T) & =c\left(E_{1}\right)+c\left(E_{2}\right)+c\left(E_{3}\right) \\
& \leqslant 4 a_{4}+3 a_{3}+2\left(a_{4}+a_{3}\right)+2\left(|R|-\left(4 a_{4}+3 a_{3}\right)\right) \\
& =6 a_{4}+5 a_{3}+2 a_{2},
\end{aligned}
$$

onde $a_{2}:=|R|-\left(4 a_{4}+3 a_{3}\right)$.

Observe também que o número de terminais em $R$ pode ser expresso por

$$
4 b_{4}+3 b_{3}+2 b_{2}+b_{1}=4 a_{4}+3 a_{3}+a_{2} \text {. }
$$

Portanto, se consideramos os denominadores diferentes de zero, temos que

$$
\begin{aligned}
\frac{c(T)}{c\left(T^{*}\right)} & \leqslant \frac{6 a_{4}+5 a_{3}+2 a_{2}}{5 b_{4}+4 b_{3}+3 b_{2}+2 b_{1}} \\
& =\frac{6 a_{4}+5 a_{3}+2\left(4 b_{4}+3 b_{3}+2 b_{2}+b_{1}-4 a_{4}-3 a_{3}\right)}{5 b_{4}+4 b_{3}+3 b_{2}+2 b_{1}} \\
& =\frac{8 b_{4}+6 b_{3}+4 b_{2}+2 b_{1}-2 a_{4}-a_{3}}{5 b_{4}+4 b_{3}+3 b_{2}+2 b_{1}} \\
& \leqslant \max \left\{\frac{8 b_{4}+6 b_{3}-2 a_{4}-a_{3}}{5 b_{4}+4 b_{3}}, \frac{4 b_{2}}{3 b_{2}}, \frac{2 b_{1}}{2 b_{1}}\right\} \\
& \leqslant \frac{17}{12}
\end{aligned}
$$

onde a desigualdade (3.13) segue das desigualdades (3.10) e (3.11), a igualdade (3.14) segue da igualdade (3.12) e a última desigualdade segue do lema 3.10.

Uma observação final é que ainda podemos melhorar ligeiramente a razão $17 / 12$ do lema 3.10 para um valor assintoticamente próximo a 38/27, ou seja,

$$
1,407 \ldots=\frac{38}{27}<\frac{17}{12}=1,4166 \ldots
$$

Primeiro, observe que queremos minimizar $\left(8 b_{4}+6 b_{3}-2 a_{4}-a_{3}\right) /\left(5 b_{4}+4 b_{3}\right)$ da expressão (3.15). Se conseguirmos que $2 a_{4}+a_{3} \geqslant 26 b_{4} / 27+10 b_{3} / 27$, então temos que

$$
\begin{aligned}
\frac{8 b_{4}+6 b_{3}-2 a_{4}-a_{3}}{5 b_{4}+4 b_{3}} & \leqslant \frac{(8-26 / 27) b_{4}+(6-10 / 27) b_{3}}{5 b_{4}+4 b_{3}} \\
& \leqslant \max \left\{\frac{(8-26 / 27) b_{4}}{5 b_{4}}, \frac{(6-10 / 27) b_{3}}{4 b_{3}}\right\} \\
& =\frac{38}{27}
\end{aligned}
$$


Suponha que $b_{4} \geqslant b_{3}$. Aplicamos então o algoritmo de Hurkens \& Schrijver (1989) para obter um empacotamento $\mathcal{A}^{\prime}$ com

$$
a_{4}^{\prime} \geqslant \frac{b_{4}}{2+\varepsilon^{\prime}}
$$

conjuntos de tamanho 4 , que é estendido de forma gulosa adicionando $a_{3}^{\prime}$ conjuntos de tamanho 3 de $\mathcal{C}$ que podem ser empacotados. Usando a equação (3.9) temos que

$$
4 a_{4}^{\prime}+3 a_{3}^{\prime} \geqslant 2 b_{4}+b_{3}
$$

ou seja,

$$
\frac{4 a_{4}^{\prime}}{3}+a_{3}^{\prime} \geqslant \frac{2 b_{4}}{3}+\frac{b_{3}}{3} .
$$

Usando o empacotamento obtido, temos que

$$
\begin{aligned}
\frac{4 a_{4}^{\prime}}{3}+\frac{\left(2+\varepsilon^{\prime}\right) a_{4}^{\prime}}{3}+a_{3}^{\prime} & \geqslant \frac{2 b_{4}}{3}+\frac{b_{4}}{3}+\frac{b_{3}}{3} \\
\left(2+\frac{\varepsilon^{\prime}}{3}\right) a_{4}^{\prime}+a_{3}^{\prime} & \geqslant b_{4}+\frac{b_{3}}{3} \\
& =\frac{26}{27} b_{4}+\frac{b_{4}}{27}+\frac{b_{3}}{3} \\
& \geqslant \frac{26}{27} b_{4}+\frac{10}{27} b_{3} .
\end{aligned}
$$

Na última desigualdade usamos que $b_{4} \geqslant b_{3}$.

Se, por outro lado, supomos que $b_{3} \geqslant b_{4}$, aplicamos o algoritmo de Hurkens \& Schrijver (1989) para $\mathcal{C}$ restrito a conjuntos de tamanho 3, para obter um empacotamento $\mathcal{A}^{\prime \prime}$ com

$$
a_{3}^{\prime \prime} \geqslant \frac{2\left(b_{4}+b_{3}\right)}{3+\varepsilon^{\prime \prime}}
$$

conjuntos de tamanho 3. Portanto,

$$
\begin{aligned}
a_{3}^{\prime \prime} & \geqslant \frac{2 b_{4}}{3+\varepsilon^{\prime \prime}}+\frac{2 b_{3}}{3+\varepsilon^{\prime \prime}} \\
& =\frac{2 b_{4}}{3+\varepsilon^{\prime \prime}}+\frac{8 b_{3}}{27+9 \varepsilon^{\prime \prime}}+\frac{10 b_{3}}{27+9 \varepsilon^{\prime \prime}} \\
& \geqslant \frac{26 b_{4}}{27+9 \varepsilon^{\prime \prime}}+\frac{10 b_{3}}{27+9 \varepsilon^{\prime \prime}} .
\end{aligned}
$$

Na última desigualdade usamos que $b_{3} \geqslant b_{4}$.

Fazendo os dois empacotamentos e escolhendo como resultado o empacotamento que maximiza $2 a_{4}+a_{3}$, podemos refazer as contas da equação (3.15) para obter

$$
\frac{8 b_{4}+6 b_{3}-2 a_{4}-a_{3}}{5 b_{4}+4 b_{3}} \leqslant \frac{38}{27}+\varepsilon
$$

O valor de $\varepsilon$ pode ser escolhido arbitrariamente pequeno. Porém, quanto menor esse valor, maior será tempo de processamento do algoritmo de Hurkens \& Schrijver (1989) para obtenção dos empacotamentos. 


\subsubsection{Algoritmo Terminal $_{1,2}$}

Apresentamos a seguir uma 17/12-aproximação para o problema $\mathrm{TST}_{1,2}$ e a prova do teorema 3.7. O ALGORITMO TERMINAL $L_{1,2}$, como já comentamos, escolhe um vértice de Steiner, executa duas reduções na instância de entrada e realiza um empacotamento dos terminais. Esses passos geram uma árvore de Steiner de terminais folhas com um vértice de Steiner escolhido como raiz. Repetimos então esse processo para cada vértice de Steiner da instância.

ALGORITMO TERMINAL 1,2 : recebe um grafo completo $G$, uma função custo $c: E_{G} \rightarrow\{1,2\}$ e um conjunto $R \subseteq V_{G}$ de terminais, e devolve uma árvore de Steiner de terminais folhas.

1: para cada $u$ em $V_{G} \backslash R$ faça

2: $\quad$ Construa um conjunto $R_{1}$ usando a REDUÇÃo 1 sobre a instância $(G, c, R, u)$.

3: Construa os conjuntos $R_{2}$ e $S$ usando a REDUÇ $\tilde{A} O 2$ sobre a instância $\left(G, c, R \backslash R_{1}\right)$.

4: Construa uma árvore de Steiner de terminais folhas $T_{u}^{\prime \prime}$ usando o ALGORITMO EMPACOTA sobre a instância $\left(G, c, R \backslash\left(R_{1} \cup R_{2}\right), u\right)$.

5: Construa uma árvore de Steiner de terminais folhas $T_{u}^{\prime}$ para $\left(G, c, R \backslash R_{1}\right)$ usando a árvore $\mathrm{T}_{u}^{\prime \prime}$, o conjunto $\mathrm{S}$ e o lema 3.8 .

6: Construa uma árvore de Steiner de terminais folhas $T_{u}$ para $(G, c, R, u)$ usando a árvore $\mathrm{T}_{u}^{\prime}$ e o lema 3.9.

7: Seja T uma árvore de Steiner de terminais folhas de custo mínimo entre as árvores construídas na linha 1.

8: Devolva T e pare.

Prova DO TEOREMA 3.7. Afirmamos que o ALGORITMO TERMINAL 1,2 constrói uma árvore de Steiner de terminais folhas com a razão de aproximação desejada.

$O$ algoritmo constrói, para cada $u$ em $V_{G} \backslash R$, uma árvore de Steiner de terminais folhas $T_{u}$ contendo u como um vértice de Steiner. Como uma conseqüência imediata dos lemas 3.11, 3.9, e 3.8, $c\left(T_{u}\right)$ é no máximo 17/12 vezes o custo de uma árvore de Steiner de terminais folhas ótima contendo $u$. Se a instância $G, c$ e $R$ é viável e $|R| \geqslant 3$, qualquer árvore de Steiner de terminais folhas contém pelo menos um vértice de Steiner $u$. Portanto, $T_{u}$ é a aproximação almejada para a dada instância do problema $\mathrm{TST}_{1,2}$.

\subsection{APX-completude para o problema $\mathrm{TST}_{1,2}$}

Como mencionamos antes, Lu et al. (2003) adaptaram o algoritmo de aproximação e a Lredução de Bern \& Plassmann (1989) para o problema TST 1,2 . Apresentaram então uma 8/5aproximação e mostraram que o problema TST $_{1,2}$ é MAXSNP-difícil. Essa prova é uma Lredução do problema da cobertura de vértices para o problema TST 1,2 . Nesta seção, faremos uma AP-redução do problema $\mathrm{ST}_{1,2}$ para o problema $\mathrm{TST}_{1,2}$. Essa redução nos parece mais simples e natural. 
TEOREMA 3.12 O problema TST 1,2 é APX-completo.

Prova. Nas seções 3.1, 3.2 e 3.3 mostramos que existe uma $\alpha$-aproximação para o problema TST $_{1,2}$, onde $\alpha$ é uma constante. Por isso, o problema TST 1,2 está em APX.

Para mostrar que o problema $\mathrm{TST}_{1,2}$ é APX-difícil escolhemos um problema APX-completo, o problema $\mathrm{ST}_{1,2}$, e faremos uma AP-redução do problema $\mathrm{ST}_{1,2}$ para o problema $\mathrm{TST}_{1,2}$. Ou seja, mostramos que $\mathrm{ST}_{1,2} \leqslant \mathrm{AP} \mathrm{TST}_{1,2}$. Para tanto, devemos determinar uma tripla $(f, g, \beta)$ satisfazendo (AP1), (AP2), (AP3) e (AP4), conforme definido na seção 1.2.

A função $f$ recebe uma instância $G, c$ e $R$ do problema $S T_{1,2}$ e produz uma instância $\left(G^{\prime}, c^{\prime}, R^{\prime}\right):=f(\delta,(G, c, R))$ do problema $T_{S T} T_{1,2}$ da seguinte forma: (i) toda aresta do grafo $\mathrm{G}$ que conecta dois terminais e tem custo 1 é contraída, como mostra a figura 3.11; e (ii) se dois ou mais vértices de Steiner são conectados a um vértice terminal por arestas de custo $1 \mathrm{em}$ $G$, então são adicionadas arestas de custo 1 entre esses vértices de Steiner, como mostra a figura 3.12. O grafo $G^{\prime}$ é resultado de ambas as transformações (i) e (ii) de f. Note ainda que $G^{\prime}$ é completo e pode conter arestas múltiplas. Então, para cada par de vértices de $V_{\mathrm{G}^{\prime}}$, eliminamos todas as arestas entre esses vértices, menos a aresta de menor custo. Assim, definimos a função $f$ de (AP1).

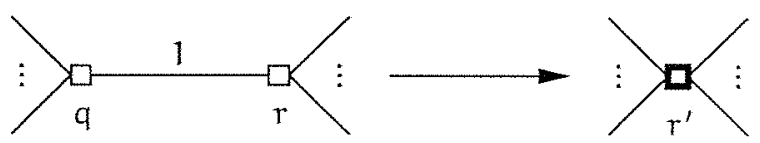

Figura 3.11: Contração de terminais ligados por uma aresta de custo 1.

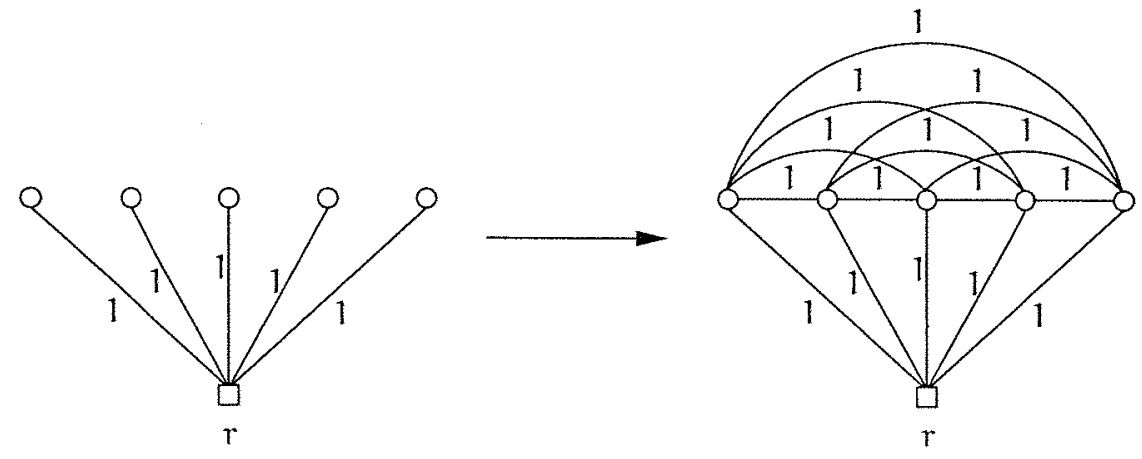

Figura 3.12: Adição de arestas entre 5 vértices de Steiner ligados ao terminal $r$.

A função $g$ recebe um racional positivo $\delta$, uma instância $G, c$ e $R$ do problema $S_{1,2}$ e uma solução $T^{\prime}$ do problema $T_{S T} T_{1,2}$ para a instância $G^{\prime}, c^{\prime}$ e $R^{\prime}$ construída pela transformação $f$, e transforma $T^{\prime} \mathrm{em} T$, uma solução para o problema $\mathrm{ST}_{1,2}$. A função $\mathrm{g}$ faz basicamente o inverso do que a função $f$ faz: (i) para cada terminal $r^{\prime}$ em $R^{\prime}$ resultante de uma contração, substitua $r^{\prime}$ por um par de vértices terminais $q, r$ em $R$ conectados por uma aresta de custo 1; e (ii) para cada aresta $e=s s^{\prime}$ em $E_{G^{\prime}}$ tal que e não pertence a $E_{G}$, substitua e por uma aresta sr em $E_{G}$, para algum terminal $r$ em R. Veja as figuras 3.13 e 3.14 . 


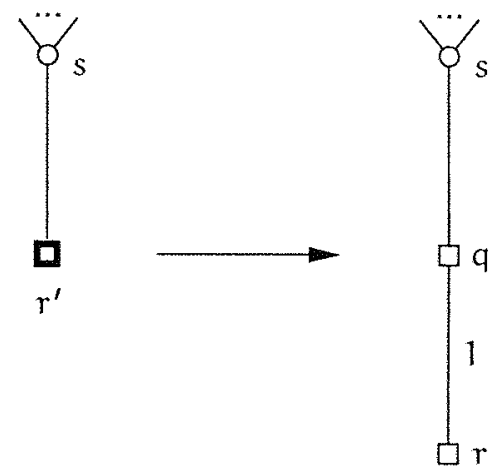

Figura 3.13: Expansão de um terminal na árvore $T^{\prime}$.
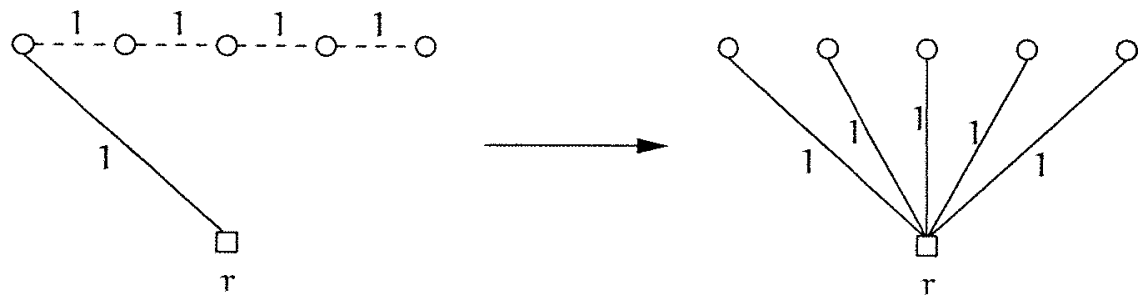

Figura 3.14: Substituições de arestas de $\mathrm{T}^{\prime}$. Arestas tracejadas não estão em $\mathrm{E}_{\mathrm{G}}$.

Portanto, a árvore T é uma solução válida para o problema $\mathrm{ST}_{1,2}$ com instância $\mathrm{G}, \mathrm{c}$ e R, e assim (AP2) é satisfeita.

É fácil ver que as funções $f$ e $g$ que acabamos de definir são transformações polinomiais sobre as suas respectivas entradas e assim (AP3) está satisfeita. A figura 3.15 mostra um esquema de funcionamento dessas transformações $f$ e $g$.

Suponha que para alguma instância $G, c, R$ do problema $S T_{1,2}$, algum racional positivo $\delta$ e alguma solução $T^{\prime}$ do problema $\operatorname{TST}_{1,2}$ para a instância $\left(G^{\prime}, c^{\prime}, R^{\prime}\right)=f(\delta,(G, c, R))$ vale que

$$
c^{\prime}\left(T^{\prime}\right) \leqslant(1+\delta) \operatorname{opt}\left(\operatorname{TST}_{1,2}(\mathrm{G}, \mathrm{c}, R)\right) .
$$

Queremos provar que a árvore $T:=g\left(\delta,(G, c, R), T^{\prime}\right)$, construída pela função $g$, é tal que

$$
c(T) \leqslant(1+\beta \delta) \operatorname{opt}(S T(G, c, R)) \text {, }
$$

para algum $\beta$ fixo. Vamos escolher para esta redução o valor de $\beta:=1$.

Da forma como as transformações $f$ e $g$ foram definidas temos que

$$
c^{\prime}\left(T^{\prime}\right)=c(T)-k
$$

onde T é uma solução para o problema $S_{1,2}$ com instância $G, c$ e R e ké o número de contrações de pares de terminais conectados por arestas de custo 1 nessa instância. Essas contrações foram realizadas pela transformação $f$, no item $(i)$, como descrevemos acima. 


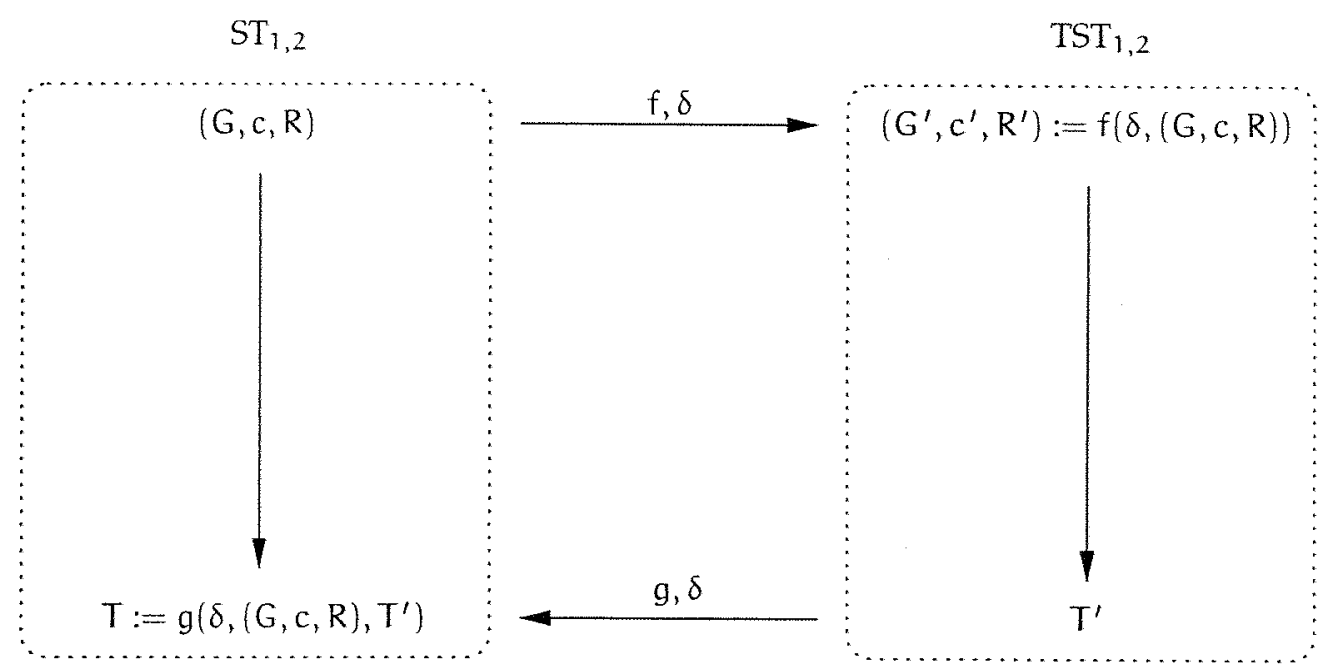

Figura 3.15: Um esquema da AP-redução do problema $\mathrm{ST}_{1,2}$ para o problema $\mathrm{TST}_{1,2}$, evidenciando as transformações $f$ e $g$.

Da igualdade (3.16), temos em particular que

$$
\operatorname{opt}\left(\operatorname{TST}_{1,2}\left(G^{\prime}, c^{\prime}, R^{\prime}\right)\right)=\operatorname{opt}\left(S T_{1,2}(G, c, R)\right)-k .
$$

Então,

$$
\begin{aligned}
c^{\prime}\left(T^{\prime}\right) & \leqslant(1+\delta) \operatorname{opt}\left(\operatorname{TST}_{1,2}\left(G^{\prime}, c^{\prime}, R^{\prime}\right)\right) \\
c(T)-k & \leqslant(1+\delta)\left(\operatorname{opt}\left(S_{1,2}(G, c, R)\right)-k\right) \\
c(T) & \leqslant(1+\delta) \operatorname{opt}\left(S_{1,2}(G, c, R)\right)-\delta k \\
& \leqslant(1+\delta) \operatorname{opt}\left(S_{1,2}(G, c, R)\right) \\
& =(1+\beta \delta) \operatorname{opt}\left(S T_{1,2}(G, c, R)\right) .
\end{aligned}
$$

As desigualdades acima seguem do fato que $\delta>0, k \geqslant 0$ e $\beta=1$. Dessa forma, (AP4) é satisfeita.

Portanto, com (AP1), (AP2), (AP3) e (AP4) satisfeitas, temos que o problema $\mathrm{ST}_{1,2}$ é APredutível ao problema $\mathrm{TST}_{1,2}$, ou seja, temos que $\mathrm{ST}_{1,2} \leqslant \mathrm{AP}_{\mathrm{TST}} \mathrm{T}_{1,2}$. Dessa forma, como o problema $\mathrm{ST}_{1,2}$ é APX-completo (Bern \& Plassmann, 1989), temos que o problema $\mathrm{TST}_{1,2}$ é APXcompleto.

Uma consequiência imediata do teorema 3.12 que acabamos de demonstrar é que não existe um esquema de aproximação polinomial para o problema $\mathrm{TST}_{1,2}$, a menos que $\mathrm{P}=\mathrm{NP}$, conforme os teoremas 1.1 e 1.4. 


\section{Capítulo 4}

\section{Problema STO}

Nos problemas TST e $\mathrm{TST}_{1,2}$ que vimos nos capítulos anteriores, a restrição que os terminais sejam folhas da árvore de Steiner construída vai ao encontro de exigências de aplicações como a construção de árvores filogenéticas. Com esse mesmo intuito, podemos formular outros tipos de restrição como, por exemplo, forçar parentescos entre certos subconjuntos de espécies, o que se traduz na imposição de que subárvores sejam completamente disjuntas na árvore de Steiner construída, havendo assim agrupamentos de subconjuntos de terminais. Dessa forma, podemos propor o seguinte problema: dados um grafo completo com custos nas arestas obedecendo a desigualdade triangular, um subconjunto de vértices terminais e uma permutação nesse subconjunto, queremos encontrar uma árvore de Steiner que 'respeita' a ordem imposta por essa permutação.

Neste capítulo, propomos um esquema de aproximação polinomial para o problema STO. Esse algoritmo recebe uma instância G, c, R e $\sigma$ do problema STO e um inteiro positivo $k$ e soluciona eficientemente o problema kSTO sobre a instância $G, c, R, \sigma$ e $k$, devolvendo uma árvore de Steiner k-restrita ótima que respeita $\sigma$. Essa árvore, como veremos, é uma aproximação para a solução do problema STO. Como conseqüência imediata, mostramos então que o problema kSTO está na classe PO e o problema STO está na classe PTAS.

\subsection{Razão k-Steiner para árvores de Steiner com ordenação nas folhas}

Nesta seção, relacionamos o custo de uma árvore de Steiner ótima que respeita uma ordenação com o custo de uma árvore de Steiner k-restrita ótima que respeita uma ordenação. Como veremos, queremos determinar o valor da seguinte razão:

$$
\sup _{(G, c, R, \sigma)}\left\{\frac{\operatorname{opt}(k \operatorname{STO}(G, c, R, \sigma, k))}{\operatorname{opt}(\operatorname{STO}(G, c, R, \sigma))}\right\} .
$$

Vamos mostrar que essa razão tem o mesmo valor da razão $k$-Steiner definida na seção 1.3, isto é, $\rho_{k}$. 
TEOREMA 4.1 Para todo inteiro não negativo $k=2^{r}+s$, com $0 \leqslant s<2^{r}$, temos que

$$
\sup _{(G, c, R, \sigma)}\left\{\frac{\operatorname{opt}(k S T O(G, c, R, \sigma, k))}{\operatorname{opt}(\operatorname{STO}(G, c, R, \sigma))}\right\}=\frac{(r+1) 2^{r}+s}{r 2^{r}+s}=\rho_{k}
$$

A prova desse teorema é baseada na prova do teorema de Borchers \& Du (1997), que determina o valor da razão k-Steiner. São pequenas as modificações realizadas nessa demonstração.

Usamos a definição usual de árvore binária como sendo um grafo conexo e acíclico onde todo vértice tem grau no máximo 3. Exemplos de árvores binárias são apresentados na figura 4.1. Confundimos então uma árvore com a representação gráfica de uma árvore e daí decorrem as definições usuais associadas aos seus vértices como raiz, pai, filho, filho esquerdo, filho direito, interno, descendente e nível de um vértice. Esses termos são ilustrados na figura 4.1(i). Uma árvore binária regular é uma árvore binária onde todo vértice interno tem dois filhos, como ilustrado na figura 4.1(ii).

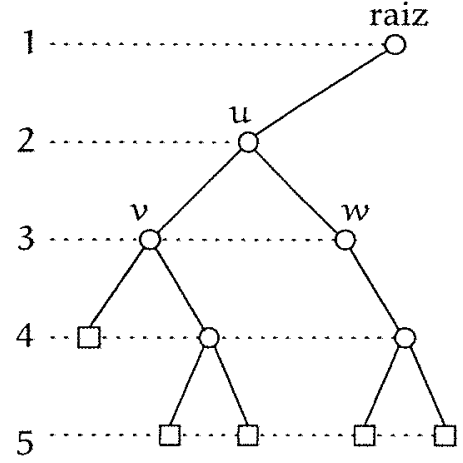

(i)

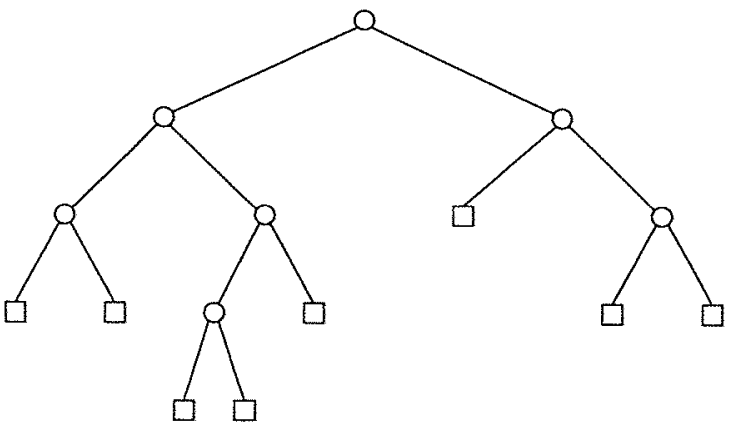

(ii)

Figura 4.1: Representações de árvores binárias. (i) Uma árvore binária com o vértice u pai dos vértices $v$ e $w$, que são filho esquerdo e direito de $u$, respectivamente; os níveis dos vértices também são destacados. (ii) Uma árvore binária regular.

Seja $T$ uma árvore binária regular e $R \subseteq V_{T}$ o conjunto de suas folhas. Observe inicialmente que T é uma árvore de Steiner de terminais folhas, com $R$ o conjunto de terminais de T. Definimos a função caminho-folha $f$ que associa cada vértice interno $u$ em $V_{T} \backslash R$ de $T$ a uma folha $f(u)$ em $R$ como segue:

(i) se u não é a raiz de T e u é filho esquerdo de seu vértice pai, determine o caminho $p_{u}$ de $u$ a uma folha $f(u)$ em $R$ tal que para todo vértice $v$ no caminho $p_{u}, v \neq u$, temos que $v$ é filho direito do pai de $v$;

(ii) se u não é a raiz de $T$ e u é filho direito de seu vértice pai, determine o caminho $p_{u}$ de $u$ a uma folha $f(u)$ em $R$ tal que para todo vértice $v$ no caminho $p_{u}, v \neq u$, temos que $v$ é filho esquerdo do pai de $v$;

(iii) se $u$ é raiz de $T$, determine o caminho $p_{u}$ de $u$ à folha $f(u)=r_{\sigma_{1}}$ ou à folha $f(u)=r_{\sigma_{\mid R\}}}$. 
Como mencionado, a definição da função f que acabamos de descrever é uma modificação da mesma função de Borchers \& Du (1997). Um exemplo de uma função f para a árvore binária da figura 4.1(ii) é apresentado na figura 4.2.

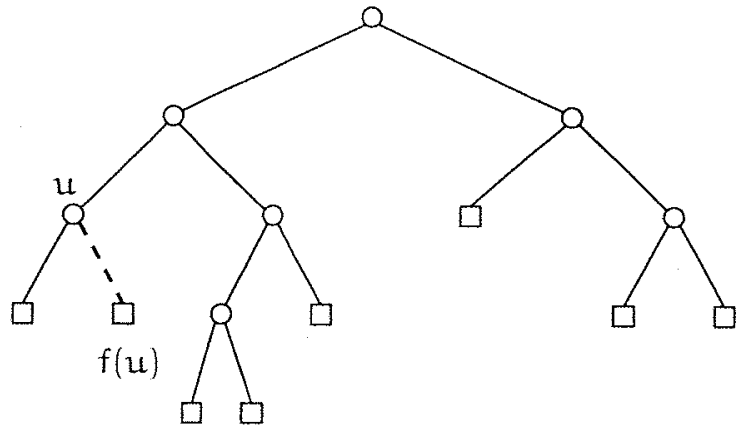

(i)

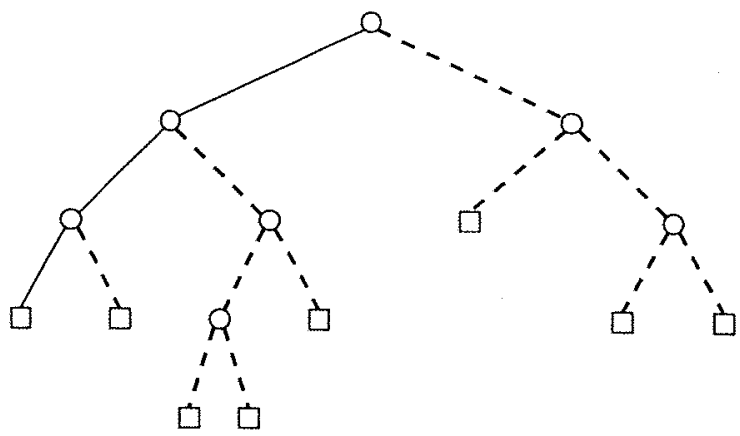

(ii)

Figura 4.2: Definição da função $f$ para a árvore binária regular da figura 4.1(ii). (i) Função $f$ definida para o vértice $u$ destacado; note que $u$ é filho esquerdo do pai de $u$. (ii) A função $f$ definida para a árvore inteira.

LEMA 4.2 Para toda árvore binária regular $\mathrm{T}$, a função caminho-folha fé tal que

(i) o vértice $f(u)$ é descendente de $u$, para todo vértice interno $u$ em $V_{\mathrm{T}} \backslash \mathrm{R}$;

(ii) para todo par de vértices internos $u, v$ em $V_{T} \backslash R$, os caminhos $p_{u}$ e $p_{v}$ definidos por $f$ são arestadisjuntos;

(iii) as folhas $r_{\sigma_{1}}$ e $r_{\sigma_{|R|}}$ em $R$ são tais que os caminhos da raiz de $T$ até $r_{\sigma_{1}}$ e $r_{\sigma_{|R|}}$, respectivamente, são aresta-disjuntos entre si e são aresta-disjuntos de todo caminho $p_{u}$, para todo vértice interno u que não é a raiz de $\mathrm{T}$.

PROVA. Faremos indução na altura da árvore T.

Se a altura da árvore é 0, então (i), (ii) e (iii) são satisfeitas trivialmente. Agora, seja T uma árvore binária regular de altura $h \geqslant 1$ e raiz $u$. Então, $T$ tem duas subárvores $T_{1}$ e $T_{2}$ conectadas à raiz $u$, cada uma delas com altura no máximo $h-1$. Por indução, existem funções $f_{1}$ e $f_{2}$ sobre $T_{1}$ e $T_{2}$, respectivamente, satisfazendo (i) e (ii). Existem também folhas $r_{\sigma_{1}}$ e $r_{\sigma_{j}}$ em $T_{1}$ e $r_{\sigma_{j+1}}$ e $r_{\sigma_{|R|}}$ em $T_{2}$ satisfazendo (iii). Defina então uma função caminho-folha $f$ da seguinte forma:

$$
f(v):= \begin{cases}f_{1}(v), & \text { se } v \text { é vértice interno de } T_{1}, \\ f_{2}(v), & \text { se } v \text { é vertice interno de } T_{2} \\ r_{\sigma_{1}} \text { ou } r_{\sigma_{|R|}}, & \text { se } v=u, \text { a raiz de } T\end{cases}
$$

Então, a função caminho-folha f assim definida satisfaz (i), (ii) e (iii). 
Podemos transformar uma árvore de Steiner de terminais folhas que respeita uma ordenação em uma árvore binária regular que respeita a mesma ordenação adicionando à árvore de Steiner algumas arestas de custo 0, alguns vértices de Steiner e, ainda, posicionando a raiz sobre uma aresta escolhida. Um exemplo de uma transformação como essa é apresentado na figura 4.3 .

Seja T uma árvore de Steiner de terminais folhas para uma instância $G, c$ e R, que respeita uma ordenação $\sigma$. Transforme $T$ em uma árvore binária regular que respeita $\sigma$ de mesmo custo, conforme acabamos de decrever e apresentamos na figura 4.3. Seja um inteiro não negativo $k=2^{r}+s$, onde $0 \leqslant s<2^{r}$. Os vértices internos de $T$ podem ser rotulados com subconjuntos do conjunto $\left\{1, \ldots, r 2^{r}+s\right\}$ contendo $2^{r}$ elementos, de forma recursiva. O rótulo de um vértice é determinado pelos rótulos de seus $r$ vértices ancestrais imediatos. Apresentamos a seguir um algoritmo que determina essa rotulação.

ALGORITMO ROTULAÁRVORE: recebe uma árvore binária regular $T$ com conjunto de folhas $R \subseteq V_{T}$ e um inteiro positivo $k=2^{r}+s, 0 \leqslant s<2^{r}$, e devolve $T$ com rótulos em seus vértices internos satisfazendo a propriedade da disjunção.

1: Para todo $i, 1 \leqslant i \leqslant r$, rotule os vértices do $i$-ésimo nível de $T$ com o conjunto $\left\{(i-1) 2^{r}+\right.$ $\left.1, \ldots, i 2^{r}\right\}$

2: Para todo $i \geqslant r$, execute o seguintes processamentos:

Regra 1. Seja u um vértice no nível $i+1-r$ rotulado com o conjunto $S^{u}=$ $\left\{s_{1}, \ldots, s_{2^{r}}\right\}$; rotule o $j$-ésimo descendente de $u$ no nível $\mathfrak{i}+1$ com o conjunto $S_{j}^{u}=\left\{s_{j}, \ldots, s_{j+2^{r}-s-1}\right\}$, onde os índices são reduzidos $\left(\bmod 2^{r}\right)$ de forma que permanecem no intervalo de 1 a $2^{r}$.

Regra 2. Para um vértice no nível $i+1$, adicione ao conjunto que o rotula os $s$ rótulos que não estão contidos nos conjuntos que rotulam os vértices dos seus $r$ ancestrais imediatos.

3: Devolva T e pare.

A rotulação indutiva em uma árvore binária regular $T$ realizada pelo ALGORITMO ROTULAÁRVORE mantém a seguinte propriedade.

Propriedade da disjunção. Os conjuntos que rotulam $r$ vértices consecutivos em um caminho ascendente em $T$ são disjuntos entre si.

Um exemplo de uma rotulação para a árvore T da figura 4.3(ii) é apresentado na figura 4.4.

PROPOSIÇÃo 4.3 A rotulação de uma árvore binária regular T realizada pelo ALGORITMO ROTULAÁRVORE mantém a propriedade da disjunção.

PROVA. Faremos indução no número de níveis de $T$. 


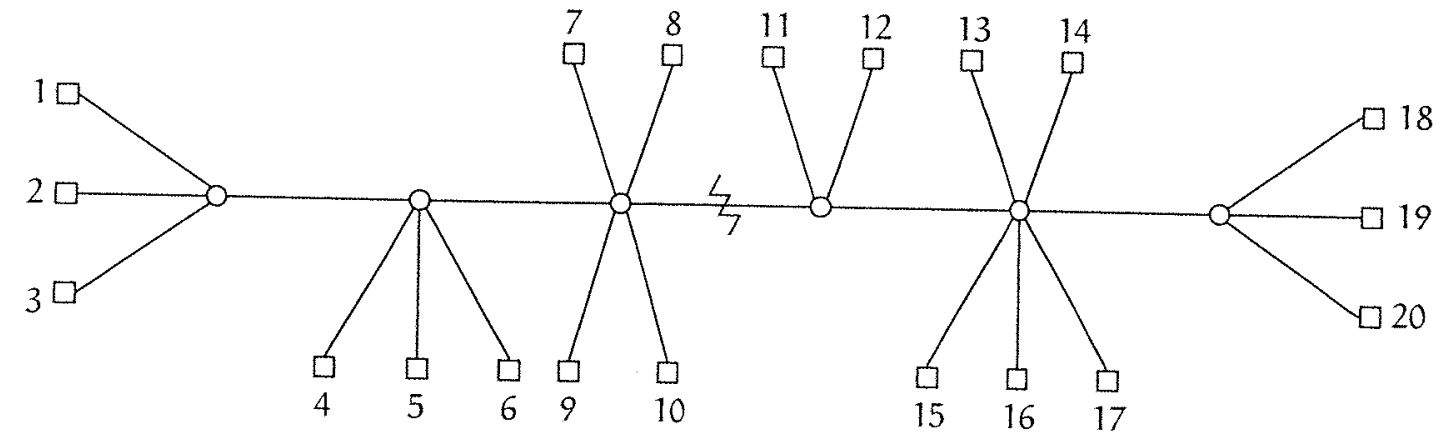

(i)

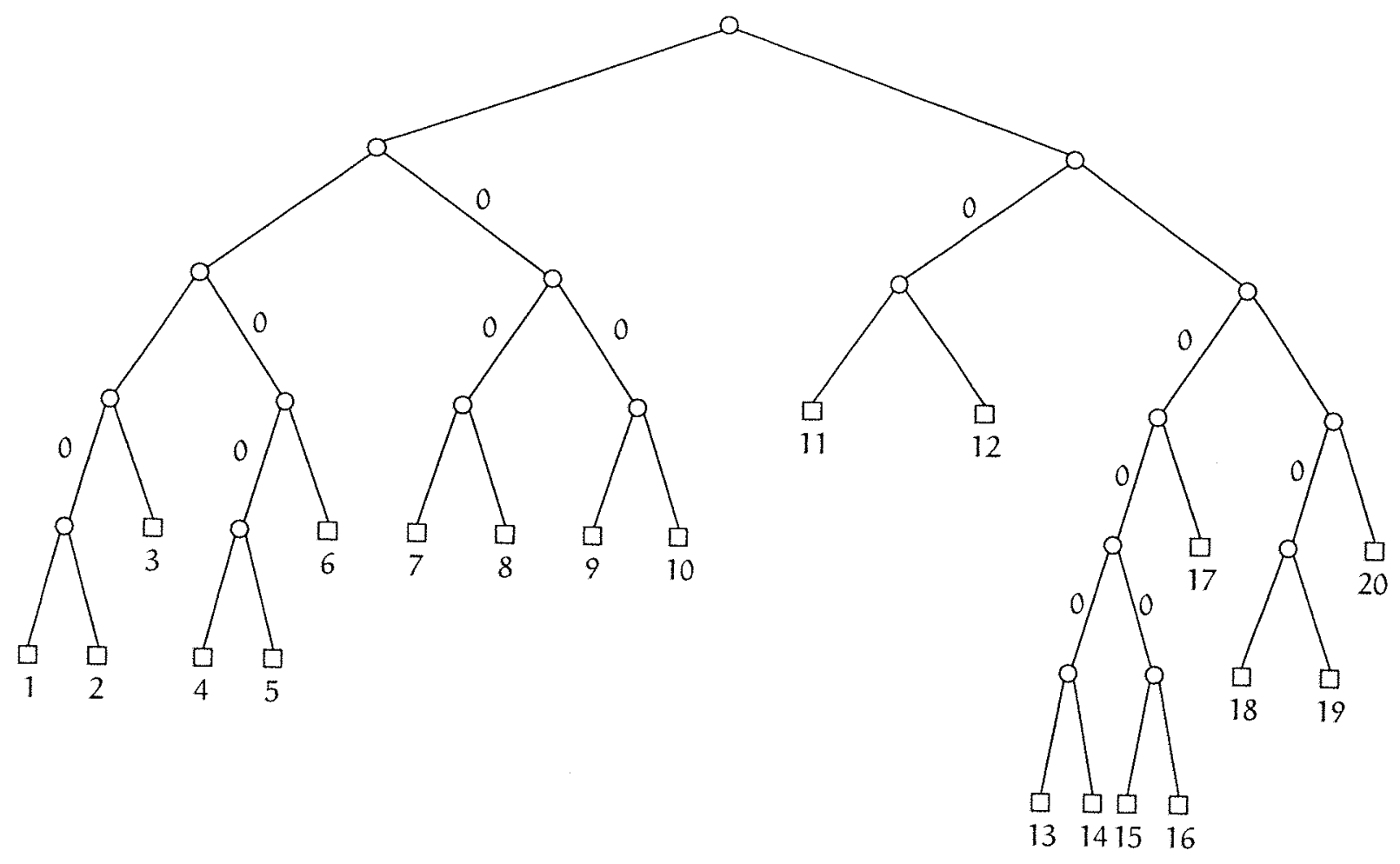

(ii)

Figura 4.3: (i) Uma árvore de Steiner de terminais folhas com custos nas arestas que respeita a ordenação $\sigma=\langle 1, \ldots, 20\rangle$. (ii) Uma árvore binária regular com raiz que respeita a ordenação $\sigma$, com mesmo custo da árvore em (i). 


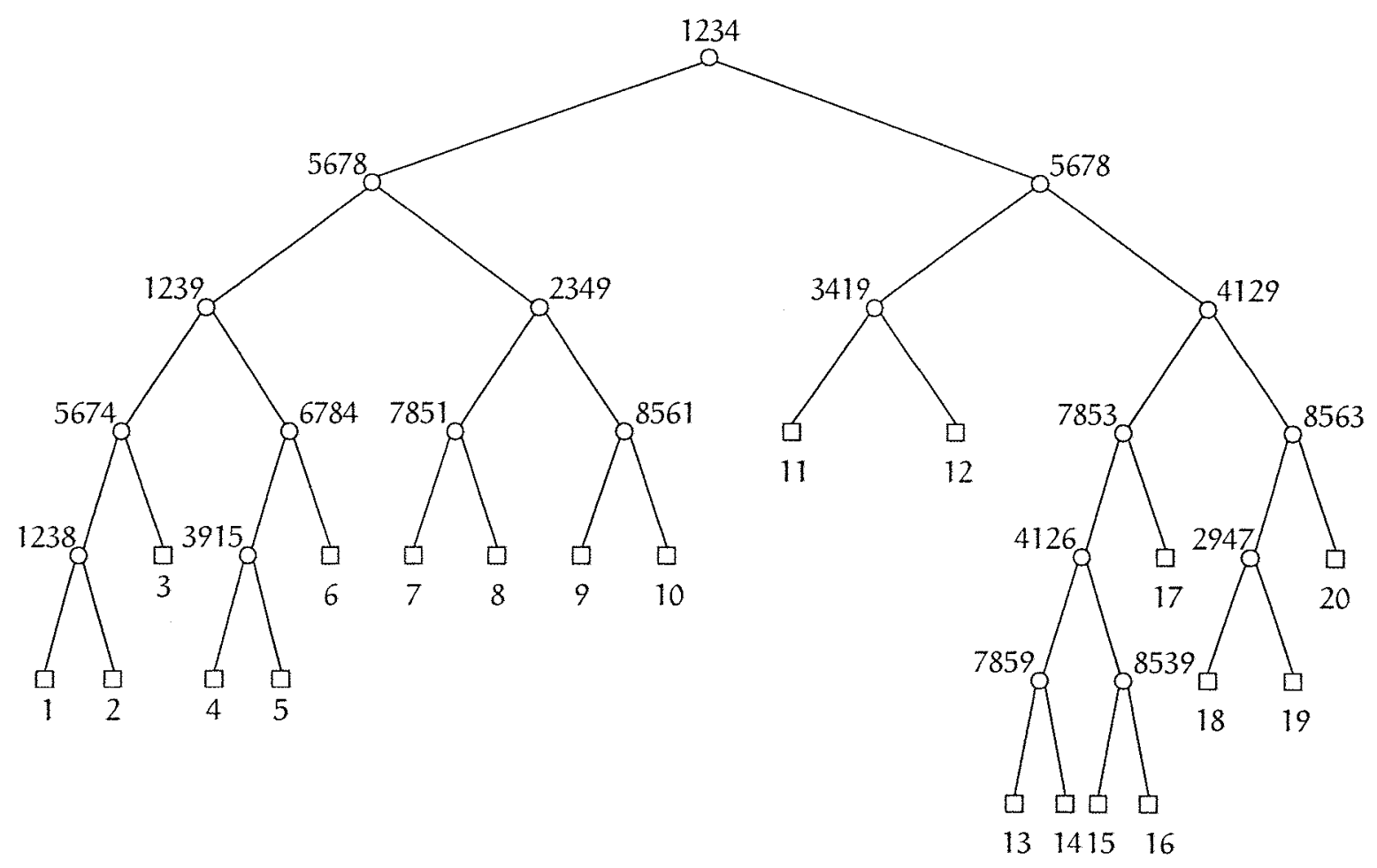

Figura 4.4: A árvore binária regular da figura 4.3(ii) com rotulação dada pelo ALGORITMO ROTULAÁRVORE.

Na primeira linha do ALGORITMO ROTULAÁRVORE, a raiz de Té rotulada com o conjunto $\left\{1, \ldots, 2^{r}\right\}$, seus filhos esquerdo e direito são rotulados com o conjunto $\left\{2^{r}+1, \ldots, 2 \cdot 2^{r}\right\}$ e, em geral, todos os vértices no i-ésimo nível de $T, 1 \leqslant i \leqslant r$, são rotulados com o conjunto $\left\{(i-1) 2^{r}+1, \ldots, i 2^{r}\right\}$. Assim, a rotulação dos $r$ primeiros níveis de $T$ claramente satisfaz a propriedade da disjunção.

Considere agora que os primeiros $i$ níveis da árvore $T, \operatorname{com} i \geqslant r$, foram rotulados e que a propriedade da disjunção se mantém até o nível i de $T$. Rotulamos então os vértices no nível $i+1$ de $T$ através das regras 1 e 2 . Pela regra 1 , um vértice interno u no nível $i+1-r$ rotulado pelo conjunto $S^{u}$ tem no máximo $2^{r}$ descendentes no nível $i+1$ e assim há a necessidade de determinar no máximo os conjuntos $S_{1}^{u}, \ldots, S_{2^{\tau}}^{u}$ no nível $i+1$. O conjunto $S_{j}^{u}$ tem $2^{r}-s$ elementos e cada elemento $s_{k}$ de $S^{u}$ está contido em no máximo $2^{r}-s$ desses conjuntos, mais precisamente em $S_{k}^{u}, \ldots, S_{k-2^{r}+s+1}^{u}$, com índices reduzidos $\left(\bmod 2^{r}\right)$. Veja a figura 4.4. Para completar a rotulação dos vértices no nível $i+1$ da árvore $T$, devemos adicionar $s$ elementos em cada conjunto definido pela regra 1. Pela propriedade da disjunção, os $r$ ancestrais imediatos de um vértice no nível $i+1$ de $T$ são rotulados por $r$ conjuntos disjuntos de tamanho $2^{r}$. Dessa forma, devem existir exatamente $s$ elementos em $\left\{1, \ldots, r 2^{r}+s\right\}$ que não foram utilizados. Além disso, os elementos adicionados aos conjuntos pela regra 2 são diferentes dos elementos adicionados pela regra 1 e assim todos os vértices no nível $i+1$ são rotulados por conjuntos de tamanho $2^{\mathrm{r}}$ pelas regras 1 e 2 . Veja a figura 4.4 para um exemplo de rotulação. 
Logo, a propriedade da disjunção é satisfeita para o nível $i+1$, já que um vértice $u$ no nível $i+1$ tem rótulo com elementos tomados do conjunto de seu r-ésimo ancestral, que pela propriedade da disjunção no nível $i$ de $T$ não são usados pelos seus $r-1$ ancestrais imediatos, e s outros elementos que também não são utilizados pelos seus $r-1$ ancestrais imediatos. Portanto, por indução no número de níveis de $T$, a rotulação determinada pelo ALGORITMO ROTULAÁRVORE mantém a propriedade da disjunção.

A partir de agora, utilizaremos a rotulação dada pelo ALGORITMO ROTULAÁRVORE sobre uma árvore binária regular $T$ com conjunto de follhas $R$ e que respeita uma ordenação $\sigma$, para construir $r 2^{r}+s$ árvores de Steiner $k$-restritas com terminais em $R$ que respeitam $\sigma$. Cada $\ell=1, \ldots, r 2^{r}+s$ determina uma árvore de Steiner $k$-restrita $T_{\ell}$, que é a união de subárvores de $T$. Alguns vértices internos e algumas arestas de $T$ são duplicados em $T_{\ell}$. A seguir, descrevemos com detalhes essas estruturas.

A determinação de uma árvore $T_{\ell}$ é realizada a partir da árvore binária regular $T$ da seguinte forma. Uma subárvore de $T$ com raiz $v$ conecta-se aos primeiros vértices descendentes de $v$ em $T$ que são rotulados por $\ell$. Esses vértices são chamados de folhas intermediárias dessa subárvore. Seguindo a composição dessa subárvore, uma folha intermediária u se conecta a uma folha $f(u)$ de $T$ através do caminho $p_{u}$, de acordo com o lema 4.2. Se não há vértices rotulados por $\ell$ em um caminho descendente em $T$ a partir de $v$, isto é, se não há folhas intermediárias nessa subárvore, então todo caminho descendente de $v$ a uma folha da árvore faz parte dessa subárvore. Finalmente, sempre existe uma subárvore de $T$ cuja raiz é a raiz da árvore $T_{\ell}$, mesmo que $\ell$ não faça parte do rótulo atribuído à raiz de $T$. Podemos enxergar a árvore $T_{\ell}$ assim definida como sendo a árvore $T$ com alguns vértices e arestas replicados, como uma junção de subárvores de $T$ que se ligam pelas suas folhas. Veja a figura 4.5 para um exemplo da decomposição em subárvores da árvore binária regular $T$ da figura 4.4 e a árvore $T_{\ell}$, para $\ell=1$, como a junção dessas subárvores na figura 4.6.

LEMA 4.4 Seja $T$ uma árvore binária regular com conjunto de folhas $\mathrm{R} \subseteq \mathrm{V}_{\mathrm{T}}$. Para todo $\ell=$ $1, \ldots, r 2^{r}+s, T_{\ell}$ é uma árvore que conecta todas as folhas em $\mathrm{R}$.

Prova. Por indução na altura de T.

Se a altura da árvore $T$ é 0 , o lema segue trivialmente. Considere a subárvore $X$ de $T_{\ell}$ que contém a raiz de $T$. Considere então uma subárvore $Y$ de $T_{\ell}$ cuja raiz é um vértice duplicado de uma folha intermediária da subárvore $X$ de $T_{\ell}$. Veja a figura 4.7. Por indução, sabemos que $Y$ é uma árvore que conecta todas as folhas em $R$ restritas a essa subárvore. A subárvore $X$ em $T_{\ell}$ conecta uma folha de $R$ para cada subárvore abaixo de suas folhas intermediárias. Isso conecta todas as folhas em $T_{\ell}$ e a indução termina.

Agora vamos mostrar que $T_{\ell}$ é uma árvore de Steiner $k$-restrita. A figura 4.5 mostra a decomposição das subárvores de $T$ da figura 4.3 , que são as subárvores de $T_{1}$. Observe que essas subárvores são árvores de Steiner k-restritas, com $k=5$. 

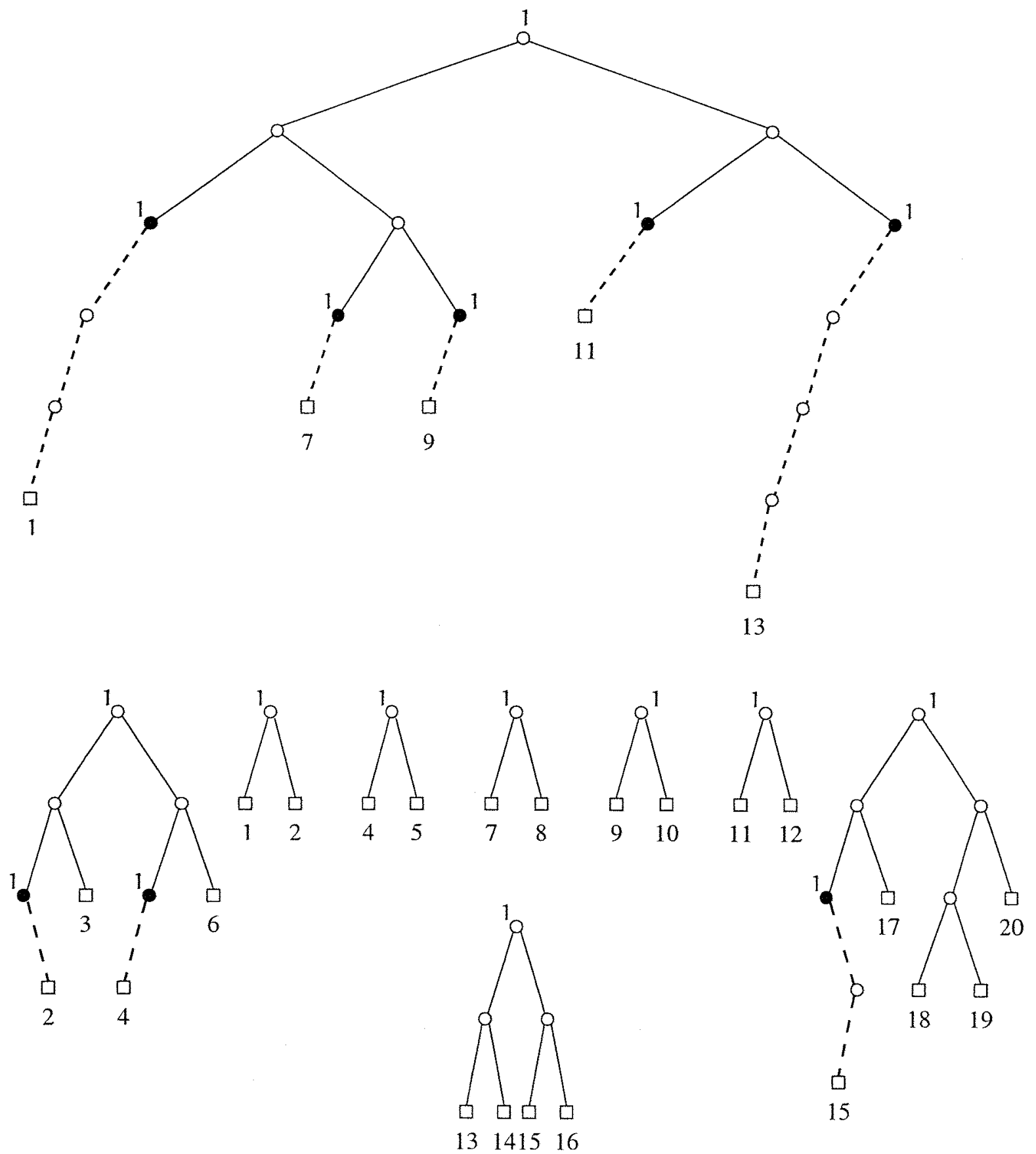

Figura 4.5: A decomposição em subárvores da árvore da figura 4.4, para $\ell=1$. Vértices internos preenchidos são folhas intermediárias da subárvore. 


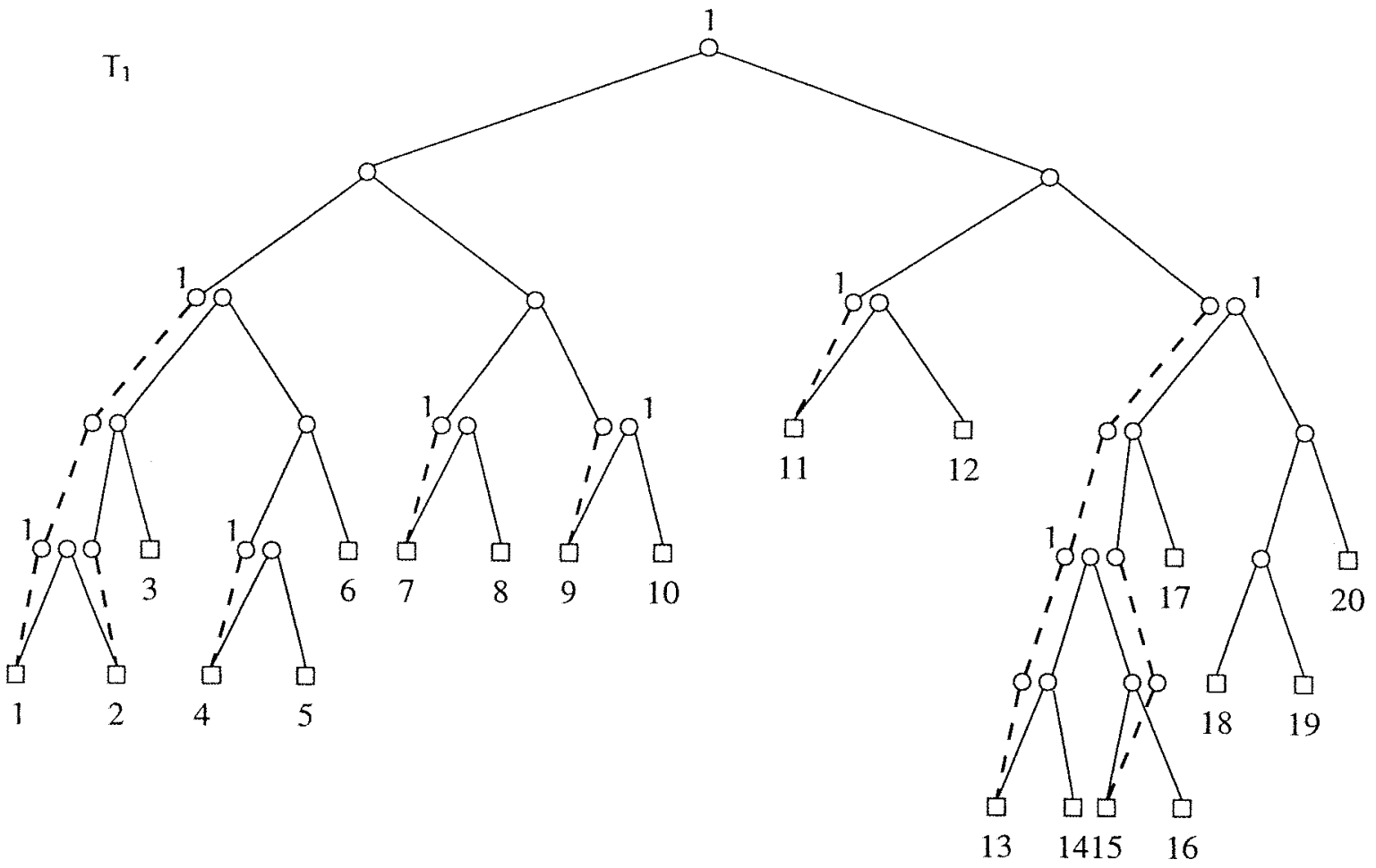

Figura 4.6: Árvore $T_{1}$ obtida da árvore da figura 4.4 e da decomposição da figura 4.5.

LEMA 4.5 Para todo $\ell=1, \ldots, \mathrm{r} 2^{\mathrm{r}}+\mathrm{s}$, a árvore $\mathrm{T}_{\ell}$ é uma árvore de Steiner $\mathrm{k}$-restrita, $\operatorname{com} \mathrm{k}=2^{\mathrm{r}}+\mathrm{s}$ $e 0 \leqslant s<2^{r}$.

Prova. Tome uma subárvore de $T_{\ell}$ com raiz $v$. Suponha que o vértice $v$ não é rotulado por $\ell$. Então, $v$ é a raiz de $T_{\ell}$ e $\ell \geqslant 2^{r}+1$. Pela linha 1 do ALGORITMO ROTULAÁrVORE, os elementos $2^{r}+1, \ldots, r 2^{r}$ aparecem nos rótulos de todos os vértices de um dos $r-1$ níveis abaixo da raiz e, pela regra 2 do algoritmo, os s elementos restantes aparecem nos rótulos de todos os vértices do r-ésimo nível abaixo da raiz. Assim, as folhas intermediárias da subárvore estão todas no $r$-ésimo nível ou acima. Portanto, a subárvore de raiz $v$ é no máximo $2^{r}$-restrita, e $2^{r} \leqslant k$. Suponha agora que o vértice $v$ é rotulado por $\ell$. Pela regra 1 no ALGORITMO ROTULAÁrVORE, sabemos que $2^{r}-s$ descendentes de $v, r$ níveis abaixo de $v$, são rotulados por $\ell$ e os $s$ descendentes restantes não são rotulados por $\ell$. Considere o vértice $u$ que está $r$ níveis abaixo de $v$ e que não é rotulado por $\ell$. Como $\ell$ rotula $v$, pela propriedade da disjunção, $\ell$ não pode rotular qualquer dos $r-1$ vértices no caminho de $u$ a $v$. Como $\ell$ não rotula $u$, pela regra 2 , os filhos de u devem ser rotulados por $\ell$ e, assim, esses vértices são folhas intermediárias dessa subárvore. Logo, a subárvore com raiz $v$ tem $2 s$ folhas intermediárias $r+1$ níveis abaixo de $v$ e $2^{r}-s$ folhas intermediárias $r$ níveis abaixo de $v$. Então, o total de folhas intermediárias é $2^{r}+s=k$ e assim a subárvore é k-restrita. Portanto, todas as subárvores de $T_{\ell}$ são k-restritas e, como as conexões são realizadas pelos vértices terminais, $T_{\ell}$ é uma árvore de Steiner k-restrita. 


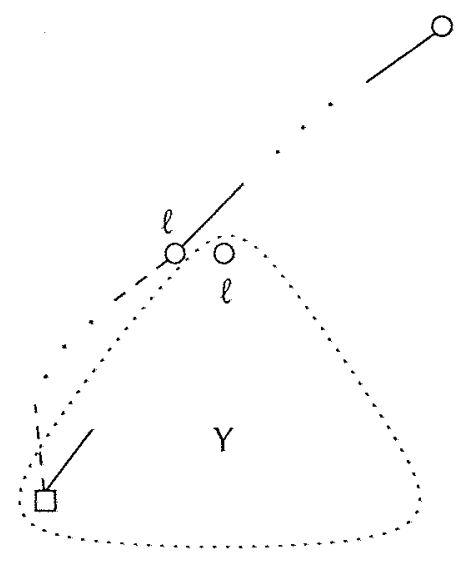

Figura 4.7: Uma subárvore $Y$ da árvore $T_{\ell}$.

LEMA 4.6 Para todo inteiro positivo $\mathrm{k}$, com $\mathrm{k}=2^{\mathrm{r}}+\mathrm{s} e \mathrm{e} \leqslant \mathrm{s}<2^{\mathrm{r}}$, temos que

$$
\frac{\operatorname{opt}(k S T O(G, c, R, \sigma))}{\operatorname{opt}(\operatorname{STO}(G, c, R, \sigma))} \leqslant \frac{(r+1) 2^{r}+s}{r 2^{r}+s} \text {. }
$$

Prova. Faremos indução em $|R|$.

Se $|R| \leqslant k$ então a desigualdade é verdadeira já que

$$
\frac{\operatorname{opt}(k \operatorname{STO}(G, c, R, \sigma, k))}{\operatorname{opt}(\operatorname{STO}(G, c, R, \sigma))}=1 \leqslant \frac{(r+1) 2^{r}+s}{r 2^{r}+s}
$$

Para $|R|>k$, considere $T$ a árvore de Steiner de custo mínimo para $G, c$ e $R$ que respeita uma ordenação $\sigma$. Se T não é uma árvore de Steiner de terminais folhas, então podemos subdividi-la em um vértice terminal que não é folha, em duas árvores de Steiner sobre dois conjuntos de terminais $R_{1}$ e $R_{2}$, que respeitam as subseqüências $\sigma_{1}$ e $\sigma_{2}$ de $\sigma$, respectivamente. Temos então que

$$
\operatorname{opt}(\operatorname{STO}(G, c, R, \sigma))=\operatorname{opt}\left(\operatorname{STO}\left(G, c, R_{1}, \sigma_{1}\right)\right)+\operatorname{opt}\left(\operatorname{STO}\left(G, c, R_{2}, \sigma_{2}\right)\right)
$$

e

$$
\operatorname{opt}(k S T O(G, c, R, \sigma, k)) \leqslant \operatorname{opt}\left(k S T O\left(G, c, R_{1}, \sigma_{1}, k\right)\right)+\operatorname{opt}\left(k S T O\left(G, c, R_{2}, \sigma_{2}, k\right)\right) .
$$

Logo,

$$
\begin{aligned}
\frac{\operatorname{opt}(k S T O(G, c, R, \sigma, k))}{\operatorname{opt}(\operatorname{STO}(G, c, R, \sigma))} & \leqslant \frac{\operatorname{opt}\left(\mathrm{kSTO}\left(G, c, R_{1}, \sigma_{1}, k\right)\right)+\operatorname{opt}\left(\mathrm{kSTO}\left(G, c, R_{2}, \sigma_{2}, k\right)\right)}{\operatorname{opt}\left(\operatorname{STO}\left(G, c, R_{1}, \sigma_{1}\right)\right)+\operatorname{opt}\left(\operatorname{STO}\left(G, c, R_{2}, \sigma_{2}\right)\right)} \\
& \leqslant \max \left\{\frac{\operatorname{opt}\left(\mathrm{kSTO}\left(G, c, R_{1}, \sigma_{1}, k\right)\right)}{\operatorname{opt}\left(\operatorname{STO}\left(G, c, R_{1}, \sigma_{1}\right)\right)}, \frac{\operatorname{opt}\left(\mathrm{kSTO}\left(G, c, R_{2}, \sigma_{2}, k\right)\right)}{\operatorname{opt}\left(\operatorname{STO}\left(G, c, R_{2}, \sigma_{2}\right)\right)}\right\} \\
& \leqslant \frac{(r+1) 2^{r}+s}{r 2^{r}+s},
\end{aligned}
$$

por hipótese de indução. 
Considere então T uma árvore de Steiner de custo mínimo para G, c e R que respeita uma ordenação $\sigma$ tal que T é uma árvore de Steiner de terminais folhas.

Transforme T em uma árvore binária regular, como na figura 4.3. Rotule os vértices internos de T através do ALGORITMO ROTULAÁRVORE. A partir de T rotulada, construa $r 2^{r}+s$ árvores de Steiner $k$-restritas, com $k=2^{r}+s$, conforme as figuras 4.5 e 4.6 e o lema 4.5 .

Para todo $\ell=1, \ldots, r 2^{r}+s$, defina $C_{\ell}$ como a soma dos custos dos caminhos $p_{u}$ das folhas intermediárias $u$ para as folhas $f(u)$ em toda subárvore de $T_{\ell}$. Como cada vértice interno $u$ em $T$ é uma folha intermediária em exatamente $2^{r}$ das árvores de Steiner $k$-restritas, mais precisamente $T_{\ell}$ para cada $\ell$ no conjunto que rotula $u$, o custo do caminho $p_{u}$ é computado exatamente $2^{r}$ vezes no somatório. Da forma como $f$ foi definida, esses caminhos são disjuntos para folhas intermediárias diferentes, e assim a soma de todos os custos dos caminhos em todos os termos do somatório será no máximo $2^{r}$ vezes maior que o custo da árvore $T$, que é o custo da árvore de Steiner de terminais folhas de custo mínimo para G, c, R e $\sigma$. Logo,

$$
\sum_{\ell=1}^{r 2^{r}+s} C_{\ell} \leqslant 2^{r} c(T)=2^{r} \operatorname{opt}(\operatorname{STO}(G, c, R, \sigma)) \text {. }
$$

Pela desigualdade acima, deve existir um índice $d$, com $1 \leqslant d \leqslant r 2^{r}+s$, tal que

$$
C_{d} \leqslant\left(\frac{2^{r}}{r 2^{r}+s}\right) \operatorname{opt}(S T O(G, c, R, \sigma))
$$

O custo de $\mathrm{T}_{\mathrm{d}}$ é a soma dos custos de suas subárvores k-restritas. Para uma subárvore dessa decomposição, devemos computar a soma dos custos das arestas dos caminhos da sua raiz até as suas folhas intermediárias, mais a soma dos custos das arestas dos caminhos das folhas intermediárias até as folhas da subárvore conectadas pela função f. Os caminhos das raízes das subárvores às folhas intermediárias cobrem a árvore de Steiner de terminais folhas que respeita $\sigma$ de custo mínimo $T$ exatamente uma vez e, assim, essa parcela tem custo $c(T)$. A outra parcela tem custo $C_{d}$. Portanto,

$$
c\left(T_{d}\right)=C_{d}+c(T) \leqslant\left(1+\frac{2^{r}}{r 2^{r}+s}\right) c(T)=\left(1+\frac{2^{r}}{r 2^{r}+s}\right) \operatorname{opt}(S T O(G, c, R, \sigma)) .
$$

Como opt $(k S T O(G, c, R, \sigma, k)) \leqslant c\left(T_{d}\right)$, pelas inequações 4.1 e 4.2 temos finalmente que

$$
\frac{\operatorname{opt}(k S T O(G, c, R, \sigma, k))}{\operatorname{opt}(\operatorname{STO}(G, c, R, \sigma))} \leqslant \frac{c\left(T_{d}\right)}{\operatorname{opt}(S T O(G, c, R, \sigma))} \leqslant\left(1+\frac{2^{r}}{r 2^{r}+s}\right) \leqslant \frac{(r+1) 2^{r}+s}{r 2^{r}+s} \text {. }
$$

LEMA 4.7 Para todo inteiro positivo $k$, onde $k=2^{r}+s e 0 \leqslant s<2^{r}$, temos que

$$
\sup _{(G, c, R, \sigma)}\left\{\frac{\operatorname{opt}(k S T O(G, c, R, \sigma, k))}{\operatorname{opt}(S T O(G, c, R, \sigma))}\right\} \geqslant \frac{(r+1) 2^{r}+s}{r 2^{r}+s} .
$$


PROVA. Se esse limitante inferior é menor para alguma instância $G, c, R, \sigma$ e $k$ dos problemas kSTO e STO, esse resultado tem implicação imediata sobre o limitante inferior de $\rho_{k}$ estabelecido por Borchers \& Du (1997).

Observe finalmente que o resultado principal dessa seção, o teorema 4.1, segue imediatamente dos lemas 4.6 e 4.7 .

\subsection{Uma $\rho_{k}$-aproximação para o problema STO}

Nesta seção propomos um algoritmo de aproximação para o problema STO. Esse algoritmo estabelece o problema STO na classe de complexidade PTAS, já que é um esquema de aproximação polinomial. Esse algoritmo constrói uma árvore de Steiner k-restrita ótima para a instância do problema STO e um inteiro positivo k. O problema da árvore de Steiner k-restrita que respeita uma ordenação é então estabelecido na classe de complexidade PO.

A técnica de programação dinâmica é incorporada no algoritmo para construção da árvore de Steiner k-restrita de custo mínimo para a instância G, c, R, $\sigma$ e k. Qualquer árvore de Steiner $k$-restrita $T$ que respeita $\sigma$ pode ser decomposta de uma das seguintes maneiras. Na primeira delas, a árvore $T$ pode ser subdividida em duas subárvores $T_{1}$ e $T_{2}$ tais que, para algum $l$, $1<l<|R|$, a subárvore $T_{1}$ contém os terminais $r_{\sigma_{1}}, r_{\sigma_{2}}, \ldots, r_{\sigma_{1}}$, enquanto a árvore $T_{2}$ contém os terminais $r_{\sigma_{1}}, r_{\sigma_{l+1}}, \ldots, r_{\sigma_{|R|}}$. Veja a figura 4.8 para uma ilustração. O custo da árvore T é a soma dos custos de $T_{1}$ e $T_{2}$. Na segunda maneira, a árvore $T$ contém uma subárvore k-restrita $T^{\prime}$ conectando $k$ terminais em $R$, para algum $\kappa, 2 \leqslant k \leqslant k$. Em particular, os terminais $r_{\sigma_{1}}$ e $r_{\sigma_{|R|}}$ são conectados por $T^{\prime}$. Os $k$ vértices conectados por $T^{\prime}$ subdividem os terminais em $\kappa-1$ intervalos. Cada intervalo I tem um início e um fim denotados por $c_{\mathrm{I}}$ e $f_{\mathrm{I}}$. Cada um desses intervalos precisa ser conectado por no máximo duas subárvores conforme ilustrado na figura 4.9. O custo de $T$ é, neste caso, a soma do custo da árvore $T^{\prime}$ mais o custo das árvores que conectam os terminais nos intervalos mencionados. No que segue, usamos a notação $\sigma_{i . . j}$ para denotar $\left\langle\sigma_{i}, \ldots, \sigma_{j}\right\rangle$.

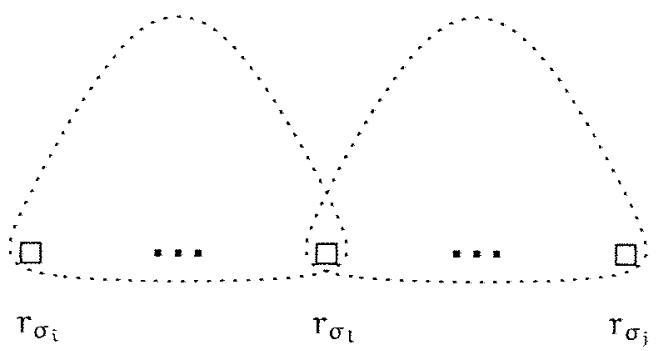

Figura 4.8: Uma possível decomposição de uma árvore $k$-restrita que respeita $\sigma_{i . . j}$, onde ocorre uma subdivisão dos terminais em um índice $l$.

Para decidir qual das duas maneiras $T$ pode ser decomposta, basta determinarmos o maior índice $i$ para o qual o vértice $r_{\sigma_{l}}$ atinge, na árvore $T$, o vértice $r_{\sigma_{i}}$ através de caminhos que 


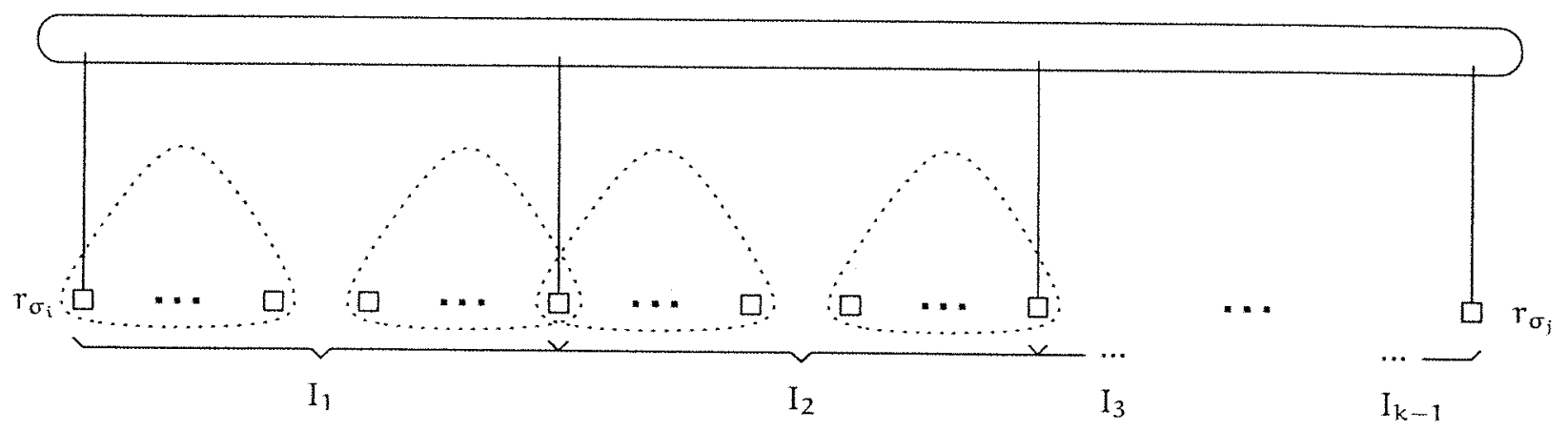

Figura 4.9: Uma outra possível decomposição de uma árvore $k$-restrita que respeita $\sigma_{\dot{i} . . j}$, onde uma componente $k$-restrita é conectada a $k$ terminais.

usam somente vértices de Steiner como vértices internos. Se $i<|R|$, T pode ser decomposta da primeira maneira. Caso contrário, a decomposição pode ser feita da segunda maneira.

Podemos definir agora o valor de uma solução ótima recursivamente em termos das soluções ótimas dos subproblemas. Seja $v$ uma matriz de programação dinâmica, tal que cada posição $(i, j)$ de $v$ contém o custo da árvore de Steiner k-restrita ótima para os terminais $\left\langle r_{\sigma_{i}}, \ldots, r_{\sigma_{j}}\right\rangle$ que respeita $\sigma_{i . . j}$. Seja $Q$ o conjunto de subconjuntos de índices de $R$ de cardinalidade pelo menos 2 e no máximo $k$. Para cada $q \in Q$, seja $T_{q}$ uma árvore de Steiner k-restrita ótima que respeita $\sigma$ restrita a q. Cada q em $Q$ subdivide os terminais num conjunto de intervalos $J_{q}$, sendo que intervalos consecutivos em $J_{q}$ se interceptam nos elementos de $R$ com índices em q. Temos então que

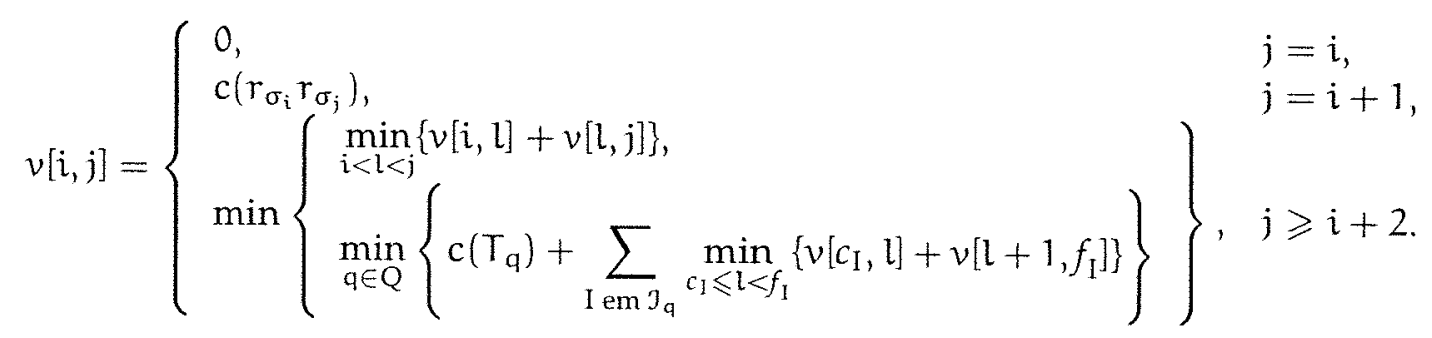

TEOREMA 4.8 A fórmula definida para $v[i, j], 1 \leqslant i \leqslant j \leqslant|R|$, computa corretamente o custo de uma árvore de Steiner k-restrita ótima que respeita $\sigma_{i . . j}$ para uma instância $G, c, R, \sigma e k$ do problema $\mathrm{kSTO}$.

Prova. Na fórmula são analisadas todas as possíveis decomposições das árvores de Steiner krestritas que respeitam $\sigma_{i . . j}$. Portanto, a decomposição da árvore ótima também é examinada. Por indução, o valor encontrado para cada uma das decomposições é ótimo, resultando na corretude da fórmula.

Dada uma instância $G, c, R$ e $\sigma$ do problema STO e um inteiro positivo $k$, podemos computar o valor de uma árvore $k$-restrita ótima que respeita $\sigma$ através do ALGORITMO VALORÁRVORE que apresentamos a seguir. 


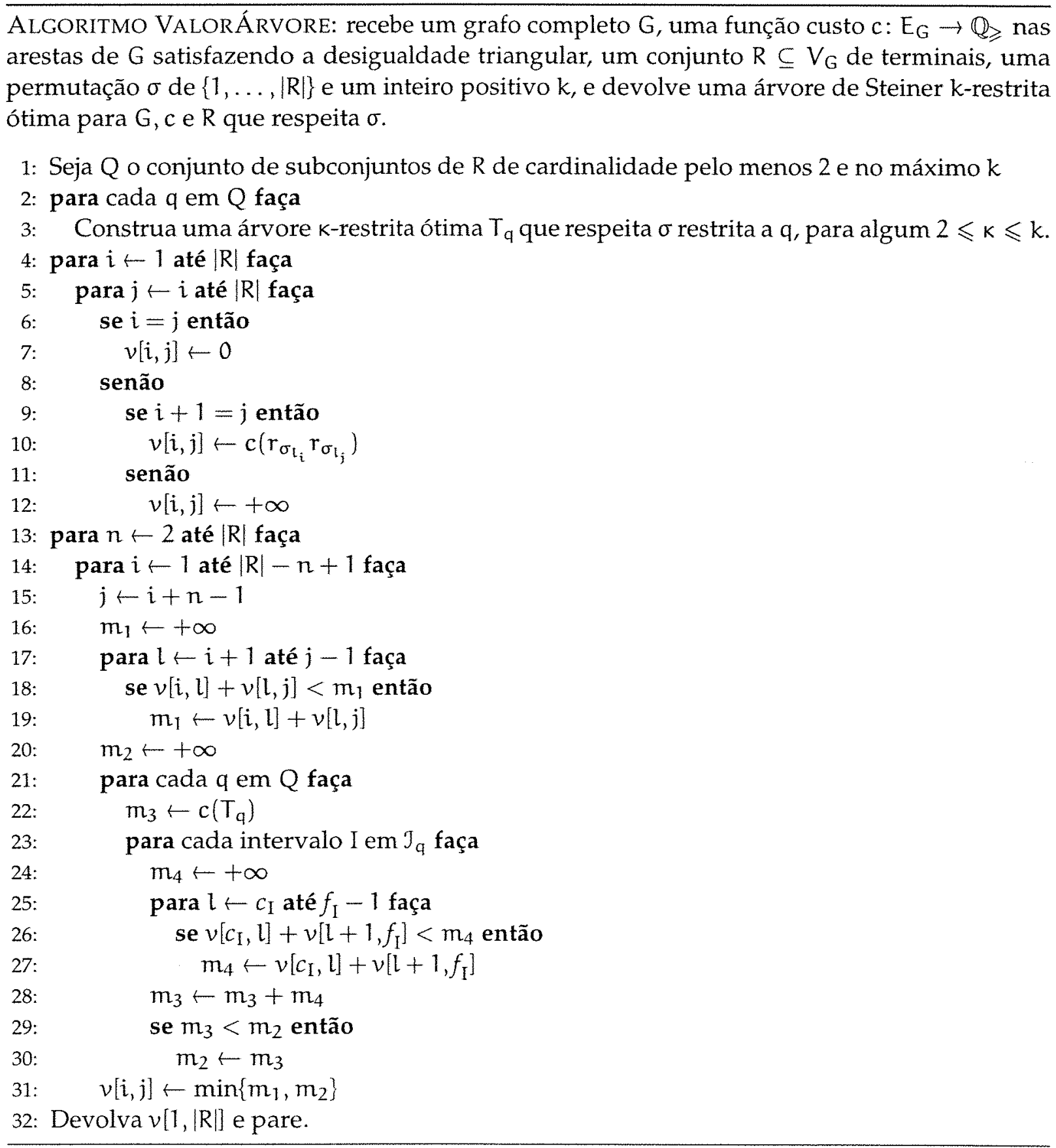


Pelo teorema 4.8, o ALGORITMO VALORÁRVORE implementa o cálculo da fórmula $v[1,[R]]$ que contém o custo de uma árvore de Steiner k-restrita ótima que respeita $\sigma$, para uma instância $G, c, R, \sigma$ e $k$ do problema kSTO. Por simples inspeção, sabemos que o tempo de execução do ALGORITMO VALORÁRVORE é $O\left(|S|^{k+1}+|R|^{k+1}\right)$, onde $S$ é o conjunto de vértices de Steiner de $\mathrm{G}$, isto é $S:=V_{\mathrm{G}} \backslash R$. Como $R \cup S=V_{\mathrm{G}}$ temos então que o tempo de execução do ALGORITMO VALORÁRVORE é $\mathrm{O}\left(\left|\mathrm{V}_{\mathrm{G}}\right|^{2 k+2}\right)$. Como $k$ é uma constante, o algoritmo é polinomial no tamanho da entrada. Dessa forma, o problema kSTO está na classe PO.

COROLÁRIO 4.9 O problema kSTO está na classe de complexidade PO.

Além disso, o ALGORITMO VALORÁRVORE é polinomial para todo k fixo e portanto o problema STO está na classe PTAS.

COROLÁRIO 4.10 O ALGORITMO VALORÁRVORE é uma $\left(1+\varepsilon_{\mathrm{k}}\right)$-aproximação para o problema STO, onde $k=2^{r}+s, 0 \leqslant s<2^{r} e$

$$
\varepsilon_{\mathrm{k}}=\frac{2^{r}+s}{r 2^{r}+s}
$$

Portanto, o problema STO está na classe de complexidade PTAS. 


\section{Capítulo 5}

\section{Conclusão}

Neste trabalho propomos algoritmos de aproximação, e também um algoritmo polinomial, para algumas restrições do problema de Steiner em grafos. Nesse problema geral, dados um grafo completo com custos nas arestas satisfazendo a desigualdade triangular e um subconjunto dos vértices do grafo, chamado de conjunto dos terminais, queremos encontrar uma árvore de Steiner de custo mínimo.

O problema de Steiner dos terminais folhas, ou problema TST, é uma restrição do problema de Steiner em grafos onde queremos encontrar uma árvore de Steiner de custo mínimo tal que todos terminais sejam folhas. Mostramos no capítulo 2 que o problema TST é APX-completo e propomos dois algoritmos de aproximação: o ALGORITMO TERMINAL apresentado na seção 2.2 com razão de aproximação $\alpha=\min \left\{\alpha_{1}, \alpha_{2}\right\}$, onde

$$
\alpha_{1}=1+\frac{10 \rho(\rho-1)}{7 \rho-6}, \quad \alpha_{2}=4 \rho-3
$$

e o ALGORITMO TERMINAL ${ }^{\star}$ apresentado na seção 2.3 com razão de aproximação

$$
\alpha^{\star}=2 \rho-\left(\frac{\rho}{3 \rho-2}\right)
$$

supondo que um algoritmo de aproximação de tempo polinomial obtém uma razão de aproximação $\rho \leqslant 2$ para o problema de Steiner em grafos. Como o melhor algoritmo de aproximação para o problema de Steiner em grafos, o algoritmo proposto por Robins \& Zelikovsky (2000), estabelece $\rho=1+(\ln 3) / 2 \leqslant 1,55$, temos então que o ALGORITMO TERMINAL é uma 2,76-aproximação e o ALGORITMO TERMINAL é uma 2,52-aproximação para o problema de Steiner dos terminais folhas.

No capítulo 3 tratamos do problema $\mathrm{TST}_{1,2}$, o problema de Steiner dos terminais folhas com restrição para os custos das arestas, onde os custos são 1 ou 2. Mostramos que o problema TST $_{1,2}$ é APX-completo e apresentamos o ALGORITMO TERMINAL 1,2, uma 17/12-aproximação para esse problema. 
Finalmente, no capítulo 4, trabalhamos com o problema de Steiner em grafos com uma ordenação para suas folhas, denominado problema STO. Focalizamos então o problema da árvore k-restrita com uma ordenação para suas folhas, chamado de problema kSTO. Inicialmente, mostramos que a razão k-Steiner definida para os problemas STO e kSTO tem o mesmo valor da razão $k$-Steiner defina por Borchers \& Du (1997). Em seguida, utilizamos a técnica de programação dinâmica para propor um algoritmo exato para o problema kSTO e mostramos que esse algoritmo é um esquema da aproximação polinomial para o problema STO. Ou seja, mostramos que o ALGORITMO VALORÁRVORE apresentado na seção 4.2 soluciona exatamente o problema kSTO e é uma $\rho_{k}$-aproximação para o problema STO.

Um resumo dos resultados anteriores para os problemas acima mencionados e dos resultados obtidos neste trabalho segue abaixo.

\begin{tabular}{|c|c|c|c|}
\hline \multirow{2}{*}{ PROBLEMA } & \multirow{2}{*}{ COMPLEXIDADE } & \multicolumn{2}{|c|}{ ALGORITMO } \\
\hline & & RAZÃO DE APROXIMAÇ̃̃O & REFERENCIAS \\
\hline \multirow{4}{*}{ TST } & \multirow{4}{*}{ APX-completo } & $(2+\rho)$ & (Lin \& Xue, 2002) \\
\hline & & $2 \rho$ & $\begin{array}{c}\text { (Fuchs, 2003) } \\
\text { (Chen et al., 2003) } \\
\text { (Drake \& Hougardy, 2004) }\end{array}$ \\
\hline & & $\min \left\{1+\frac{10 \rho(\rho-1)}{7 \rho-6}, 4 \rho-3\right\}$ & (seção 2.2) \\
\hline & & $2 \rho-\left(\frac{\rho}{3 \rho-2}\right)$ & (seção 2.3) \\
\hline \multirow[t]{2}{*}{$\operatorname{TST}_{1,2}$} & \multirow[t]{2}{*}{ APX-completo } & $8 / 5$ & (Lu et al., 2003) \\
\hline & & $17 / 12$ & (seção 3.3) \\
\hline kSTO & $\mathrm{PO}$ & & (seção 4.2) \\
\hline STO & PTAS & $\rho_{k}$ & (seção 4.2) \\
\hline
\end{tabular}

Sobre o problema TST, podemos pensar ainda na determinação da razão de aproximação do ALGORITMO TERMINAL ${ }^{\star}$, por acreditar que essa análise pode ser melhorada. Podemos pensar também em extensões do problema $\mathrm{TST}_{1,2}$, como o problema $\mathrm{TST}_{1, \mathrm{~d}}$, onde os custos das arestas são 1 ou d (Halldórson et al., 1998), ou o problema TST $T_{i, j}$, onde os custos das arestas são $i$ ou $j$, ou ainda problema $\operatorname{TST}_{[i, j]}$, onde os custos das arestas estão no intervalo $[i, j]$. Precisamos também implementar o algoritmo proposto para o problema STO, mesmo porque seu funcionamento é bastante restritivo quando $k$ torna-se grande. É também nossa intenção verificar se não há a possibilidade de construir um algoritmo exato eficiente para o problema STO, movendo-o para a classe $\mathrm{PO}$, ou se conseguimos classificá-lo precisamente de acordo com sua dificuldade. Além disso, podemos continuar utilizando outras técnicas para o desenvolvimento de algoritmos de aproximação para os problemas TST, TST $T_{1,2}$ e STO sempre com o objetivo de construir algoritmos com melhores razões de aproximação. 


\section{Referências Bibliográficas}

Arora, S., Lund, C., Motwani, R., Sudan, M., \& Szegedy, M. (1992). Proof verification and hardness of approximation problems. In Proceedings of the 33rd Annual IEEE Symposium on Foundations of Computer Science (FOCS) (pp. 14-23). Pitsburgh, Pennsylvania, USA: IEEE Computer Society Press.

Ausiello, G., Crescenzi, P., Gambosi, G., Kann, V., Marchetti-Spaccamela, A., \& Protasi, M. (1999). Complexity and Approximation: Combinatorial Optimization Problems and their Approximability Properties. Springer-Verlag.

Bern, M. \& Plassmann, P. (1989). The Steiner problem with edge lengths 1 and 2. Information Processing Letters, 32, 171-176.

Borchers, A. \& Du, D.-Z. (1997). The k-Steiner ratio in graphs. SIAM Journal on Computing, 26, $857-869$.

Chen, Y. H., Lu, C. L., \& Tang, C. Y. (2003). On the full and bottleneck full Steiner tree problems. In The Ninth International Computing and Combinatorics Conference-COCOON (pp. 122-129). Big Sky, MT, USA.

Chlebík, M. \& Chlebíková, J. (2002). Approximation hardness of the Steiner tree problem on graphs. In M. Penttonen \& E. M. Schmidt (Eds.), Proceedings of the Eighth Scandinavian Workshop on Algorithm Theory (SWAT), volume 2368 of Lecture Notes in Computer Science (pp. 170-179). Turku, Finland: Springer-Verlag.

Drake, D. E. \& Hougardy, S. (2004). On approximation algorithms for the terminal Steiner tree problem. Information Processing Letters, 89(1), 15-18.

Fernandes, C. G., Miyazawa, F. K., Cerioli, M. R., \& Feofiloff, P., Eds. (2001). Uma Introdução Sucinta a Algoritmos de Aproximação. Publicações Matemáticas. Instituto de Matemática Pura e Aplicada - IMPA.

Fuchs, B. (2003). A note on the terminal Steiner tree problem. Information Processing Letters, 87, 219-220.

Gilbert, E. N. \& Pollak, H. O. (1968). Steiner minimal trees. SIAM Journal of Applied Mathematics, 16, 1-29. 
Gondo, E. K. (2002). Árvores k-restritas e aproximações para o problema de Steiner em grafos. Master's thesis, Universidade de São Paulo, São Paulo.

Gröpl, C., Hougardy, S., Nierhoff, T., \& Prömel, H. J. (2001). Approximation algorithms for the Steiner tree problem in graphs. In D. Z. Du \& X. Cheng (Eds.), Steiner Trees in Industries (pp. 235-279). Dordrecht: Kluwer Academic Publishers.

Halldórson, M. M., Ueno, S., Nakao, H., \& Kajitani, Y. (1998). Approximating Steiner trees in graphs with restricted weights. Networks, 31(4), 283-292.

Hurkens, C. A. J. \& Schrijver, A. (1989). On the size of systems of sets every $t$ of which have an SDR, with an application to the worst-case ratio of heuristics for packing problems. SIAM Journal of Discrete Mathematics, 2(1), 68-72.

Lin, G. \& Xue, G. (2002). On the terminal Steiner tree problem. Information Processing Letters, $84,103-107$.

Lu, C. L., Tang, C. Y., \& Lee, R. C.-T. (2003). The full Steiner tree problem. Theoretical Computer Science, 306, 55-67.

Papadimitriou, C. H. (1994). Computational Complexity. Addison-Wesley Publishing Company.

Rayward-Smith, V. J. (1983). The computation of nearly minimal Steiner trees in graphs. International Journal of Maths, Education Science and Technology, 14(1), 15-23.

Robins, G. \& Zelikovsky, A. (2000). Improved Steiner tree approximation in graphs. In Proceedings of the 11th Annual ACM-SIAM Symposium on Discrete Algorithms (SODA) (pp. 770-779). San Francisco, California, USA.

Schrijver, A. (2003). Combinatorial optimization. Polyhedra and efficiency, volume 24A. Paths, flows, matchings of Algorithms and Combinatorics. Berlin: Springer-Verlag.

Vazirani, V. V. (2001). Approximation Algorithms. Springer-Verlag.

Zelikovsky, A. (1993). An 11/6-approximation algorithm for the Steiner tree problem in graphs. Algorithmica, 9, 463-470. 